\title{
Heat Transport Phenomena in Shallow Geothermal Boreholes
}

\section{Development of a Numerical Model and a Novel Extension for the Thermal Response Test Method by Applying Oscillating Excitations}

\author{
Dissertation \\ zur Erlangung des mathematisch-naturwissenschaftlichen Doktorgrades \\ "Doctor rerum naturalium" \\ der Georg-August-Universiät Göttingen \\ im Promotionsprogramm Geowissenschaften / Geographie \\ der Georg-August University School of Science (GAUSS)
}

\author{
vorgelegt von \\ Phillip Oberdorfer \\ aus Göttingen
}

Göttingen, 2014 


\section{Betreuungsausschuss:}

Prof. Dr. Martin Sauter, Abt. Angewandte Geologie, Universität Göttingen

PD Dr. Ekkehard Holzbecher, Abt. Angewandte Geologie, Universität Göttingen

\section{Mitglieder der Prüfungskommission}

\section{Referent:}

Prof. Dr. Martin Sauter, Abt. Angewandte Geologie, Universität Göttingen

Korreferent:

PD Dr. Ekkehard Holzbecher, Abt. Angewandte Geologie, Universität Göttingen

weitere Mitglieder der Prüfungskommission:

Prof. Dr. Günter Buntebarth, Institut für Geophysik, TU Clausthal

Dr. Iulia Ghergut, Abt. Angewandte Geologie, Universität Göttingen

Prof. Dr. Andreas Tilgner, Institut für Geophysik, Universität Göttingen

Prof. Dr. Ugur Yaramanci, Leibniz-Institut für Angew. Geophysik, Hannover

Tag der mündlichen Prüfung: 21. Februar 2014 


\section{Abstract}

The branch of shallow geothermics is part of renewable energies with a potential that has not been fully exploited. Although various analytical and numerical approaches to determine the processes in vertical borehole heat exchangers (BHEs) have recently been developed, plenty of open questions about this topic remain. Also, the state-of-the-art testing routines and evaluation methods may be improved for academic purposes.

A 3-D-numerical model is developed to solve the partial differential equation systems that determine the heat-transfer phenomena in BHEs and their ambient subsurface parameters. This model is based on the finite element method and takes into account the temperature dependencies of the accompanying materials. Furthermore, it provides a simplification method to calculate the heat transfer within the BHE heat pipes, which is based on correlation solutions. The most common BHE design types (single-U, double$\mathrm{U}$, and coaxial) may be implemented easily. The single- $\mathrm{U}$ and double- $\mathrm{U}$ approaches are comprehensively validated with experimental data. The advantages of the full 3-D model are exploited by performing parametric studies including material and geometric properties as well as groundwater flow to examine the influence of different conditions on the performance of BHEs.

The validated numerical model is used to study a novel extension approach of the thermal response test, the constant heat injection step is overlapped by an oscillatory injection rate and the system response, in terms of the pipe fluid temperature, is evaluated. The results of experimental in situ test series are evaluated and numerical parametric studies are performed, to interpret the thermal reaction of BHEs on oscillating excitations. The oscillation data is found to provide further information about the borehole quality and the subsurface ambient parameters. 


\section{Acknowledgements}

This dissertation was carried out at the Georg-August University in Göttingen and funded by the European Union, the Federal State of Lower Saxony and the GeorgAugust University (EFRE, W2-8011 4860), which are gratefully acknowledged.

I would like to thank gratefully Prof. Dr. Martin Sauter for this opportunity and for his encouragement and help along the way. Also, my sincere gratitude to PD Dr. Ekkehard Holzbecher, whose support, assistance and many discussions have helped my research to progress and improve and guided me through this process.

Thanks to the supervisory committee, Prof. Dr. Günter Buntebarth, Dr. Iulia Ghergut, Prof. Dr. Andreas Tilgner and Prof. Dr. Ugur Yaramanci for their time and interest in my work.

I am grateful to the research partners of the Geo-Solar-WP project, a special thanks to the ISFH partners for the interesting and inspiring cooperation.

Likewise, I am grateful to my friends and colleagues Rui, Frieder and Olav for the discussions, support and the great time. I am also thankful to all the other colleagues from the department.

Finally, I would like to thank all my loved ones; family, friends and especially Prisca. 


\section{Contents}

Abstract $\quad$ i

$\begin{array}{ll}\text { Acknowledgements } & \text { ii }\end{array}$

List of Figures $\quad$ v

List of Tables viii

$\begin{array}{ll}\text { Abbreviations } & \text { ix }\end{array}$

Symbols

List of Publications $\quad$ xiv

1 Introduction $\quad 1$

1.1 Closed Loop Shallow Geothermal Systems . . . . . . . . . . . . . . . . . . 2

1.2 State of Knowledge . . . . . . . . . . . . . . . . . . . . . 4

1.2 .1 BHE Models . . . . . . . . . . . . . . . . . . . 4

1.2.2 Thermal Response Tests . . . . . . . . . . . . . . . . 6

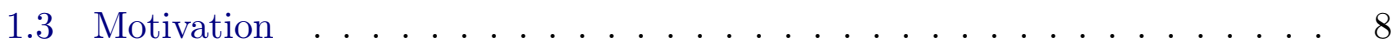

2 Theoretical Background $\quad \mathbf{1 0}$

2.1 Heat Transfer in Porous Media . . . . . . . . . . . . . . . . . . . . . 11

2.2 Heat Transfer in Borehole Heat Exchangers . . . . . . . . . . . . . . . . . 12

2.2.1 Convective Heat Transfer in Pipes . . . . . . . . . . . . . . . . . . 14

2.3 Thermal Response Tests . . . . . . . . . . . . . . . . . . . 17

3 Methods $\quad \mathbf{2 2}$

3.1 Experimental Test Site . . . . . . . . . . . . . . . . . . 22

3.2 Numerical Implementation . . . . . . . . . . . . . . . . . . . . 25

3.2 .1 Mesh Verification . . . . . . . . . . . . . . . . . . . 29

4 Results $\quad 31$

4.1 Model Validations . . . . . . . . . . . . . . . . . . . . . 31

4.1 .1 Type Curves . . . . . . . . . . . . . . . . . . . 31

4.1.2 Comparison to an Experimental Sandbox Setup . . . . . . . . . . . 40

4.1 .3 Discussion . . . . . . . . . . . . . . . . . . 43

4.2 Numerical Studies . . . . . . . . . . . . . . . . . . . . . 45 
4.2 .1 Parametric Variations . . . . . . . . . . . . . . . . . . . . 45

4.2 .2 Influence of Groundwater Flow . . . . . . . . . . . . . . . . . . . 52

4.2 .3 Discussion . . . . . . . . . . . . . . . . . . 54

5 Oscillatory Thermal Response Tests $\quad \mathbf{5 6}$

5.1 Background . . . . . . . . . . . . . . . . . 56

5.2 Oscillatory Heat Injection in BHEs . . . . . . . . . . . . . . . . . . 59

5.3 Experimental Set Up . . . . . . . . . . . . . . . . . . . . . . . . . . . . . . 61

5.3 .1 Results ........................ 63

5.4 Numerical Studies . . . . . . . . . . . . . . . . . . . . 6 68

5.4.1 Comparison to the Long-Period Analytical Solution . . . . . . . . 71

5.4 .2 System Response Studies . . . . . . . . . . . . . . . . . 73

5.5 Discussion . . . . . . . . . . . . . . . . . . . 77

6 Concluding Remarks $\quad \mathbf{8 0}$

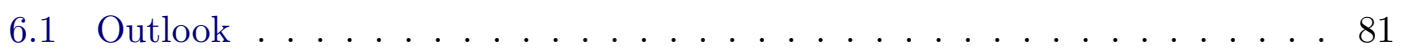

$\begin{array}{lr}\text { A Nusselt Number Correlations } & 83\end{array}$

B Temperature Dependence of the Working Fluid $\quad 87$

$\begin{array}{lr}\text { C O-TRT Data Post-Processing } & 89\end{array}$

$\begin{array}{ll}\text { Bibliography } & 91\end{array}$ 


\section{List of Figures}

1.1 Common BHE designs . . . . . . . . . . . . . . . . . . . . 4

2.1 Example for coupling methods between different PDEs . . . . . . . . . . . 12

2.2 Cross-sectional sketch of the velocity field in a pipe for different regimes, modified after Incropera et al. [2011] . . . . . . . . . . . . . . . . . 14

2.3 Comparison of different pipe flow correlations $N u(R e)$ in the transient regime, $\operatorname{Pr}=9$; (1) Gnielinski [1976], (2) Huber and Schuler [1997], (3) GVC-VDI [2006] (German Engineer Association, Heat Atlas), (4) + (5) Dittus and Boelter $[1930] \ldots \ldots \ldots \ldots$

2.4 Temperature dependence of $R e$ for different flow rates, for the example of a water-antifreeze mix. . . . . . . . . . . . . . . 16

2.5 Temperature profile (qualitative) of a pipe fluid in heat exchange with the subsurface ambiance, here: heat injection . . . . . . . . . . . 17

3.1 Map of the ISFH test field, from Pärisch et al. [2011]. The BHE drillings SN (north), SO (east) and SW (west) are located in a triangular arrangement. TW denotes a temperature measurement borehole, BM and BS are pumping wells with hydraulic contact to the groundwater. . . . . . . 23

3.2 Subsurface layers at the ISFH test site from Pärisch et al. [2011], modeled after LBEG. . . . . . . . . . . . . . . . . . . . . 25

3.3 Different domains of the 3D Single-U pipe model . . . . . . . . . . . . 26

3.4 Solution and grid visualization for different radial resolutions (very coarse to very fine) of the Double-U geometry. The right boundaries of the images are the symmetry planes of the models, only one half of the model geometry has to be considered. . . . . . . . . . . . . . . . 29

3.5 Increase of the computation time in dependence of the number of degrees of freedom for a $5[\mathrm{~h}]$-heat-injection Double- $\mathrm{U}$ simulation. The mesh refinement is executed in radial (a) and vertical (b) direction. . . . . . . . 30

3.6 Influence of the mesh size on the outflow temperature at a constant heat injection Double-U simulation after $400[\mathrm{~s}](\mathrm{a}, \mathrm{b})$ and $5[\mathrm{~h}](\mathrm{c}, \mathrm{d})$. The mesh refinement is executed in radial $(\mathrm{a}, \mathrm{c})$ and vertical $(\mathrm{b}, \mathrm{d})$ direction. . . . . 30

4.1 Fluid temperatures for down- and up-flow branches at different flow rates in the Double-U BHE model . . . . . . . . . . . . . . . . . . . . 33

4.2 Heat transfer rates between up- and down-flow pipes at different flow rates in the Double-U model . . . . . . . . . . . . . . . . . . 33

4.3 Heat transfers between the borehole and the subsurface heat at different flow rates in the Double-U model . . . . . . . . . . . . . . . . 34 
4.4 Fluid temperature for down- and up-flow branches at different times and $\mathrm{u}=0.1[\mathrm{~m} / \mathrm{s}]$ in the Double- $\mathrm{U}$ BHE model. The injection temperature is

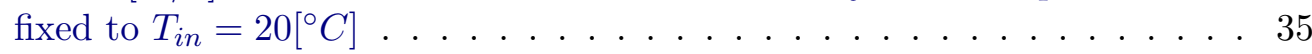

4.5 Fluid temperature for down- and up-flow branches at different times and $\mathrm{u}=0.5[\mathrm{~m} / \mathrm{s}]$ in the Double- $\mathrm{U}$ BHE model. The injection temperature is

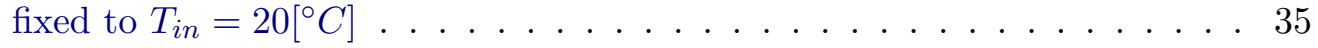

4.6 Development of integral normal boundary heat transfer of up- and downflow pipes at different flow rates in the Double-U model. . . . . . . . . . 36

4.7 Comparison of fluid temperature profiles for constant (dashed lines) and temperature dependent (solid lines) fluid parameters and different inletoutlet temperature pitches $\Delta T$, after $24[h]$ of heat injection . . . . . . 37

4.8 Differences of the outlet temperatures between constant and temperature dependent fluid parameter studies for different inlet-outlet temperature

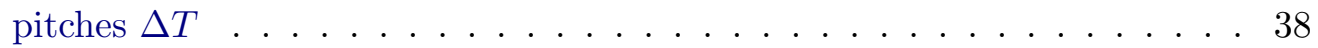

4.9 Comparison of the fluid temperature profiles of different BHE types after $3[h]$ of fluid injection with a temperature of $T_{i n}=0\left[{ }^{\circ} \mathrm{C}\right]$, from Oberdorfer et al. [2011] . . . . . . . . . . . . . . . . . . . . . 39

4.10 Numerical model geometry, front plane with symmetry boundary conditions 40

4.11 Heat injection powers for the constant and interrupted TRT . . . . . . . . 41

4.12 Mean fluid temperatures of the sandbox setup and numerical model for the constant heat injection run . . . . . . . . . . . . . . . 42

4.13 Mean fluid temperatures of the sandbox setup and numerical model for the interrupted heat injection run . . . . . . . . . . . . . . . 42

4.14 Deviation $T_{\text {num }}-T_{\text {exp }}$ between numerical model results and experimental data of the constant heat TRT . . . . . . . . . . . . . 43

4.15 Deviation $T_{\text {num }}-T_{\exp }$ between numerical model results and experimental data of the interrupted TRT . . . . . . . . . . . . . . . . . 44 44

4.16 Thermal resistance in dependence of the involved domain thermal conductivities; Working fluid (red), pipe material (blue) and grout filling (black) . . . . . . . . . . . . . . . . . . 4 47

4.17 Geometric quantities of the BHE that are varied in the study . . . . . . . 48

4.18 Thermal resistance in dependence of different geometric BHE proportions 50

4.19 Thermal resistance in dependence of the pipe radius . . . . . . . . . 51

4.20 Integral heat flux trough the pipe walls and through the borehole wall in dependence of the pipe radius . . . . . . . . . . . . . . 51

4.21 Temperature distribution of combined TRT - groundwater flow model. Darcy velocity: $u_{\text {Darcy }}=0.1\left[m^{-1}\right]$, heat injection rate: $P=2[k W]$, groundwater table: $30[m] \ldots \ldots \ldots \ldots . \ldots \ldots$

4.22 Time development of the fluid mean temperature for different groundwater flow velocities . . . . . . . . . . . . . . 5 53

4.23 Effective thermal resistance (due to TRT evaluation) in dependence of groundwater flow velocity . . . . . . . . . . . . . . 54

5.1 Phase gap and amplitude damping of the temperature response due to oscillatory excitation of any desired LTI system . . . . . . . . . . . 61

5.2 Comparison of the TRT and Oscillatory TRT principles . . . . . . . . . 62

5.3 Heat injection rates in the three test site BHEs (SE, SN, SW) during the O-TRT. I-VII mark periods with different excitation frequencies. . . . . . 63

5.4 In- and outflow temperatures during the O-TRT . . . . . . . . . . . . 64 
5.5 Excitation frequency vs. system response frequency of SE . . . . . . . 65

5.6 Oscillatory resistances of the experiment BHEs for different excitation period lengths (amplitude plot) . . . . . . . . . . . 67

5.7 Phase shifts of the experiment BHEs for different excitation period lengths

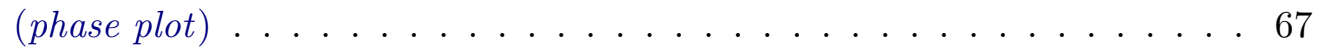

5.8 Experimental results of depth resolved temperature amplitudes and phase shifts of the three BHEs for different excitation periods $t_{0}[\mathrm{~min}] \ldots$. . . 69

5.9 Temperature screen shot: Symmetry plane cut of a model run with an injection period length of $t_{0}=10[d]$ at the point in time $t=25[d]$, which is a zero crossing point of the oscillating heat injection rate. The colour bar shows the temperature $\left[{ }^{\circ} \mathrm{C}\right] . \ldots \ldots \ldots . \ldots \ldots$

5.10 Horizontal temperature profile in x-direction through the down- and upflow branches in a depth of $z=-1[\mathrm{~m}]$ at the same state as in Figure 5.9. 71

5.11 Comparison between analytical and numerical solutions of the oscillatory thermal resistance . . . . . . . . . . . . . . . . . . 72

5.12 Comparison between analytical and numerical solutions of the signal phase shift . . . . . . . . . . . . . . . . . 73

5.13 System responses of oscillatory heat injection. The thermal conductivity of the ambient subsurface is varied between $\lambda_{s}=1-3\left[\mathrm{Wm}^{-1} \mathrm{~K}^{-1}\right]$. . . . 74

5.14 System responses of oscillatory heat injection. The heat capacity of the ambient subsurface is varied between $c_{p, s}=800-2800\left[\mathrm{Wm}^{-1} \mathrm{~K}^{-1}\right]$. . . 75

5.15 System responses of oscillatory heat injection. The thermal conductivity of the borehole grout filling material is varied between $\lambda_{g}=1-$

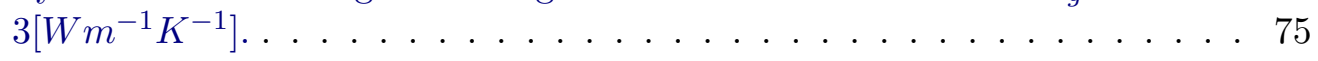

5.16 System responses of oscillatory heat injection. The heat capacity of the borehole grout filling material is varied between $c_{p, s}=800-2800\left[\mathrm{Wm}^{-1} \mathrm{~K}^{-1}\right] .76$

5.17 System responses of oscillatory heat injection. The distance between the up-flow and down-flow branches is varied between $d_{x}=36.6-128.1[\mathrm{~mm}]$ (i.e. $\left.d_{x}=0.6 \cdot d_{x 0}-2.1 \cdot d_{x 0}\right) \ldots \ldots \ldots \ldots \ldots \ldots \ldots$

5.18 System responses of oscillatory heat injection. The distance between Uloops is varied between $d_{y}=36.6-128.1[\mathrm{~mm}]$ (i.e. $\left.d_{y}=0.6 \cdot d_{y 0}-2.1 \cdot d_{y 0}\right) .77$

5.19 System responses of oscillatory heat injection. A thin thermally resistive layer is inserted between the BHE and the Subsurface domains and its resistance is varied between $R_{\text {layer }}=0.01-0.2\left[K^{2} W^{-1}\right]$. . . . . . 78

B.1 Temperature dependencies of the density and heat capacity of water (blue) and water-antifreeze mix (black) . . . . . . . . . . 87

B.2 Temperature dependencies of the thermal conductivity and dynamic viscosity of water (blue) and water-antifreeze mix (black) . . . . . . . 88

B.3 Prandtl's Number of water (blue) and water-antifreeze mix (black) as functions of temperature . . . . . . . . . . . 88

C.1 Raw signal (black), envelopes (dashed) and mean of envelopes (blue) . . . 90

C.2 Oscillatory heat injection rate $\dot{Q}_{1}(t)$, raw (black) and sine function fit (red) 90 


\section{List of Tables}

1.1 Summary of selected relevant literature contributions about TRT inverse problem solutions, following Rainieri et al. [2011] . . . . . . . . . . 8

3.1 Parameters and thermal equipment of the drillings, after Pärisch et al. [2011]. . . . . . . . . . . . . . . . . . . 24

3.2 Experimental TRT results from the ISFH test site (Voss [2011]) . . . . . 25

4.1 Parameters of the benchmark model run and variation ranges of the parametric studies . . . . . . . . . . . . . . . . 46

4.2 Fit results of the parametric studies in Figure 4.16 to the reciprocal linear function $R_{b}^{*}=\beta_{1}+\beta_{2} \lambda^{-\beta_{3}} \ldots \ldots \ldots \ldots$. . . . . . . . 48

5.1 Post-processing results of the combined oscillatory TRT . . . . . . . 66 


\section{Abbreviations}

\begin{tabular}{|c|c|}
\hline BHE & Borehole Heat Exchanger \\
\hline BM & Brunnen Mitte (well middle) \\
\hline BS & Brunnen Süd (well south) \\
\hline CSM & Cylinder Source Model \\
\hline DTS & Distributed Temperature System \\
\hline EFRE & $\begin{array}{l}\text { Europäischer Fonds für Regionale Entwicklung } \\
\text { (European Regional Development Fund) }\end{array}$ \\
\hline FEM & Finite Element Method \\
\hline GHP, GSHP & Ground Source Heat Pump \\
\hline GTRT & GeoThermal Response Test \\
\hline HDPE & High-Density PolyEthylene \\
\hline ILS & Infinite Line Source \\
\hline ISFH & $\begin{array}{l}\text { Institut für Solarenergieforschung Hameln } \\
\text { (Institute for Solar Energy Research Hamelin) }\end{array}$ \\
\hline LBEG & $\begin{array}{l}\text { Landesamt für Bergbau, Energie und Geologie } \\
\text { (State Authority for Mining, Energy and Geology) }\end{array}$ \\
\hline LHS & Left Hand Side \\
\hline LSM & Line Source Model \\
\hline LTI & Linear Time Invariant \\
\hline MSE & Mean Squared Error \\
\hline O-TRT & Oscillatory Thermal Response Test \\
\hline PDE & Partial Differential Equation \\
\hline RHS & Right Hand Side \\
\hline SN & Sonde Nord (BHE north) \\
\hline SO & Sonde Ost (BHE east) \\
\hline
\end{tabular}


SW

SX

TRT

TW

VDI
Sonde West (BHE west)

Pilot BHE

Thermal Response Test

Temperatur West

(temperature measurement borehole west)

Verein Deutscher Ingenieure

(Association of German Engineers) 


\section{Symbols}

\section{Capitals}

$\begin{array}{lll}A & \text { pipe cross sectional area } & \mathrm{m}^{2} \\ K & \text { hydraulic conductivity } & \mathrm{ms}^{-1} \\ L & \text { BHE length } & \mathrm{m} \\ N u & \text { Nusselt number } & - \\ P & \text { power / heating rate } & \mathrm{W} \\ P r & \text { Prandtl number } & - \\ Q & \text { heat source } & \mathrm{Wm}^{-3} \\ R & \text { thermal resistivity } & \mathrm{mKW}^{-1} \\ R e & \text { Reynolds number } & - \\ S & \text { specific storage } & \mathrm{m}^{-1} \\ T & \text { temperature } & \mathrm{K}^{-1} \\ \dot{V} & \text { flow-rate } & \mathrm{m}^{3} \mathrm{~s}^{-1}\end{array}$

\section{Small}

$\begin{array}{lll}a & \text { thermal diffusivity } & \mathrm{m}^{2} \mathrm{~s}^{-1} \\ c_{p} & \text { specific heat capacity } & \mathrm{Jkg}^{-1} \mathrm{~K}^{-1} \\ d & \text { thickness, distance } & \mathrm{m} \\ \mathbf{e} & \text { unit vector } & \\ h & \text { hydraulic head } & \mathrm{m} \\ \mathbf{n} & \text { normal vector } & \\ p & \text { pressure } & \mathrm{Nm}^{-2} \\ q & \text { power per unit length } & \mathrm{Wm}^{-1} \\ r & \text { radius } & \mathrm{m} \\ t & \text { time } & \mathrm{s}\end{array}$


$u$

velocity

$\mathrm{ms}^{-1}$

\section{Greek}

$\alpha$

$\beta$

$\gamma$

$\Theta$

$\kappa$

$\lambda$

$\mu$

$\nu$

$\rho$

$\omega$ heat transfer coefficient

(different) fit parameter

Euler-Mascheroni constant

volume fraction

permeability

thermal conductivity

dynamic viscosity

kinematic viscosity

density

angular frequency
$\mathrm{Wm}^{-2} \mathrm{~K}^{-1}$

$\mathrm{m}^{2}$

$\mathrm{Wm}^{-1} \mathrm{~K}^{-1}$

$\mathrm{Nsm}^{-2}$

$\mathrm{m}^{2} \mathrm{~s}^{-1}$

$\mathrm{kgm}^{-3}$

$\operatorname{rads}^{-1}$

\section{Subscripts}

$\begin{array}{ll}b & \text { borehole } \\ \text { bottom } & \text { borehole bottom } \\ \text { crit } & \text { critical } \\ \text { down } & \text { BHE down-flow branch } \\ \text { eff } & \text { effective } \\ \text { eq } & \text { equivalent } \\ \text { ext } & \text { external } \\ f, f l u i d & \text { (pipe) fluid } \\ g & \text { grout-/ back-filling } \\ i & \text { inner } \\ \text { in } & \text { BHE inlet } \\ \text { int } & \text { internal } \\ \text { o } & \text { outer } \\ \text { out } & \text { BHE outlet } \\ \text { res } & \text { pipe, periodic } \\ \text { up } & \text { resulting } \\ & \end{array}$


wall

$x, y, z$

directions (cartesian coordinate system) 


\section{List of Publications}

M.A. Rahman, P. Oberdorfer, Y. Jin, M. Pervin, and E. Holzbecher. Impact assessment of hydrologic and operational factors on the efficiency of managed aquifer recharge scheme. In Proceedings of the European COMSOL Conference in Milan, 2012. Hyperlink www.comsol.de.

P. Pärisch, O. Mercker, P. Oberdorfer, E. Bertram, R. Tepe, and G. Rockendorf. Shortterm optimization of TRNSYS models for borehole heat exchangers regarding the combination with solar heat. Renewable Energy (submitted), 2013.

P. Oberdorfer, F. Maier, and E. Holzbecher. Comparison of Borehole Heat Exchangers (BHEs): State of the Art vs. Novel Design Approaches. Proceedings of the European COMSOL Conference in Stuttgart, 2011. Hyperlink www.comsol.de.

P. Oberdorfer, R. Hu, M.A. Rahman, E. Holzbecher, M. Sauter, and P. Pärisch. Coupling Heat Transfer in Heat Pipe Arrays with Subsurface Porous Media Flow for Long Time Predictions of Solar Rechargeable Geothermal Systems. Proceedings of the European COMSOL Conference in Milan, 2012. Hyperlink www.comsol.de.

P. Oberdorfer, E. Holzbecher, R. Hu, T. Ptak, and M. Sauter. A Five Spot Well Cluster for Hydraulic and Thermal Tomography. In 38th Workshop on Geothermal Reservoir Engineering, 2013a. URL https://pangea.stanford.edu/ERE/pdf/IGAstandard/ SGW/2013/Oberdorfer1.pdf.

P. Oberdorfer, R. Hu, E. Holzbecher, and M. Sauter. A Coupled FEM Model for Numerical Simulation of Rechargeable Shallow Geothermal BHE Systems. In 38th Workshop on Geothermal Reservoir Engineering, 2013b. URL https://pangea.stanford.edu/ ERE/pdf/IGAstandard/SGW/2013/Oberdorfer . pdf. 
P. Oberdorfer. Oscillatory Thermal Response Test (OTRT) - An Advanced Method for Gaining Thermal Properties of the Subsurface. In Proceedings of the European COMSOL Conference in Rotterdam, 2013. Hyperlink www.comsol.de.

P. Pärisch, O. Mercker, P. Oberdorfer, R. Tepe, and G. Rockendorf. Experiments und Modellvalidierung für die Erdsondenregeneration mit Solarwärme. In Proceedings of the 12. Anwenderforum Oberflächennahe Geothermie, 2012.

Y. Jin, E. Holzbecher, and P. Oberdorfer. Simulation of a Novel Groundwater Lowering Technique using Arbitrary Lagrangian-Eulerian Method. In Proceedings of the European COMSOL Conference in Stuttgart, 2011. Hyperlink www.comsol.de.

E. Holzbecher, P. Oberdorfer, F. Maier, Y. Jin, and M. Sauter. Simulation of Deep Geothermal Heat Production. In Proceedings of the European COMSOL Conference in Stuttgart, 2011. Hyperlink www.comsol.de.

E. Holzbecher and P. Oberdorfer. Modelling Hydraulic and Thermal Responses in a Benchmark for Deep Geothermal Heat Production. In EGU General Assembly Conference Abstracts, volume 14, page 13829, 2012. URL http://adsabs.harvard.edu/ abs/2012EGUGA . . 1413829H.

E. Holzbecher and P. Oberdorfer. Rock Deformation due to Geothermal Heat Production - a Modelling Study. OIL GAS European Magazine (submitted), 2014.

F. Maier, P. Oberdorfer, I. Kocabas, I. Ghergut, and M. Sauter. Using temperature signals to estimate geometry parameters in fractured geothermal reservoirs. In Proceedings of the European COMSOL Conference in Milan, 2012. Hyperlink www.comsol.de.

The listed publications were released or submitted during the author's doctorate.

Last access date of all URLs: 01/19/2014 


\section{Chapter 1}

\section{Introduction}

The worldwide rise in energy consumption is supposed to be one of the major challenges of the $21^{\text {st }}$ century (UN [2010]). One one hand, a growing number of countries have succeeded in breaking the circle of poverty and underdevelopment; this is always a process accompanied by a strong rising demand for energy and, thus, the International Energy Agency (IEA) assumes an increase of 36\% from 2008 to 2035 (IEA [2013]). On the other hand, there are international efforts (UNEP [2014]) that aim to reduce the maximum worldwide mean temperature increase to $2[K]$ compared to the pre-industrial level. The containment of this temperature increase shall be realised by the reduction of carbon dioxide emissions.

According to the Renewable Energy Policy Network [2013], the worldwide share in fossil burning still amounted to $78.2 \%$ of the total energy use in 2011 . Even though the predictions of fossil reserves differ significantly, it is certain that these resources are limited. Since nuclear energy production is known to be a poor option with regards to the widely discussed risks and the problem of long-term storage of nuclear waste, it has become obvious that the current trends require the massive promotion of sustainable energies. The UNEP (United Nations Environment Programme) ranks the issue "Accelerating the Implementation of Environmentally-Friendly Renewable Energy Systems" on \#7 of their 21 Issues for the $21^{\text {st }}$ Century foresight report (UNEP [2012]).

In general, sustainable energies are defined as energies coming from sources that are replenished continuously and that are practically, from a human point of view, inexhaustible. One important source for sustainable energies is the sun. Solar energy is 
directly used by photovoltaic and solar thermal installations and indirectly by wind power systems, because the global atmospheric circulation is driven by the convective heat transport from the solar heated equator area to the cooler polar regions.

The earth itself is another source for sustainable energy production. According to recent studies by Gando et al. [2011], about 50\% of the earth's geothermal energy comes from the radioactive decay of radio-genetic isotopes, in particular uranium-238, thorium-232 and potassium-40. The other half originates from the collision processes of the original formation of the planet. However, the earth's energy potential is assumed to be significantly greater than the actual exploiting rate: Due to the IPCC Working Group III-report (Edenhofer et al. [2011]), the maximum global technical potential of geothermal heat production covers the global heat demand. However, the geothermal part of the worldwide primary energy coverage is only about 0.4\% (BMZ [2013]). One reason for the low share of geothermal energy use is the difficulty in predicting the reserves of potential locations. Usually, expensive exploration drillings are necessary and not always successful.

Worldwide geothermal resources provide energy that is transformed to electricity (about one-third) and used as direct heat (two-thirds). The electric power generation demands high temperatures that are usually found in deep formations while direct thermal extraction may also be realised in shallow depths. The largest and fastest-growing segment (20\% annually from 2005 to 2010, BMZ [2013]) of geothermics is the sub-category of shallow geothermal energy.

Shallow geothermal systems are operated in low depths, in many cases less than $100[\mathrm{~m}]^{1}$. About 80\% (GtV Bundesverband Geothermie [2013]) of the installations in Europe are closed loop shallow geothermal systems that work with vertical borehole heat exchangers.

\subsection{Closed Loop Shallow Geothermal Systems}

Geothermal heat pumps (GHP) or ground source heat pumps (GSHP) are established to use the ground as a heat source for heating purposes ${ }^{2}$. In the GSHP system, a closed

\footnotetext{
${ }^{1}$ Due to the German mining laws $(\$ 127$ BBerG), the local mining authority (Bergamt) must be considered when a borehole is to be drilled deeper than $100[\mathrm{~m}]$, which one prefers to avoid for cost saving and, perhaps, bureaucratic reasons.

${ }^{2}$ In some cases, the subsurface is also used as a heat sink and the system is used for cooling purposes or for heat storage.
} 
fluid circuit thermally connects the subsurface with a heat pump. Cold fluid is pumped through pipes that are either located in a vertical borehole or buried horizontally deep enough to avoid freezing in the winter season. After warming up by heat exchange with the subsurface, the fluid flows to the heat pump where its heat is extracted, leading to a cooling down of this fluid, before re-injection into the pipe system. Heat pumps are necessary since the temperature in shallow geothermal applications is in the range of the annual mean temperature of the location, which varis between $10-15\left[{ }^{\circ} \mathrm{C}\right]$ in Europe, i.e. the heated fluid is not hot enough for direct use, as for building heating or warm water supply. Heat pumps extract a certain amount of thermal energy and lift it up to suitable temperatures by thermodynamic processes (e.g. vaporisation and condensation).

\section{Borehole Heat Exchanger}

Borehole heat exchangers (BHEs) consist of pipes made of plastic (polypropylene (PP), polybutylene (PB), in most cases high-density polyethylene (HDPE)), situated in vertical boreholes at depths between 20 and $300[\mathrm{~m}]$ and diameters between 75 and $160[\mathrm{~mm}]$. The pipes are in general only available with certain diameters. The common types are DN 25, DN 32 or DN 40. Apart from some special designs, most pipes are arranged in a closed loop or U-loop as displayed in Figure 1.1. Using two loops (Double-U) instead of only one provides a higher specific surface to volume ratio and a lower hydraulic pressure drop. However, two loops also more expensive. The Double-U design is most frequently used in Europe while the Single-U design is preferred in North America. Another established approach is the Coaxial design, consisting of a down-flow branch that is situated inside the up-flow pipe. It is common practice to use brine or water with suitable additives as working fluid in BHEs to preserve freezing and corrosion processes within the pipes. The free space between the pipes and the borehole wall is usually filled with a certain kind of grout material to (i) provide better thermal contact between pipe and borehole ambient, to (ii) avoid the connection of horizontally layered groundwater reservoirs from different depths, which should remain hydraulically not connected with subsurface settlement, and to (iii) prevent contamination of the subsurface in case of leakage. The relative low thermal conductivity of grout is often increased by adding silica sand or graphite (Lee et al. [2010]). 


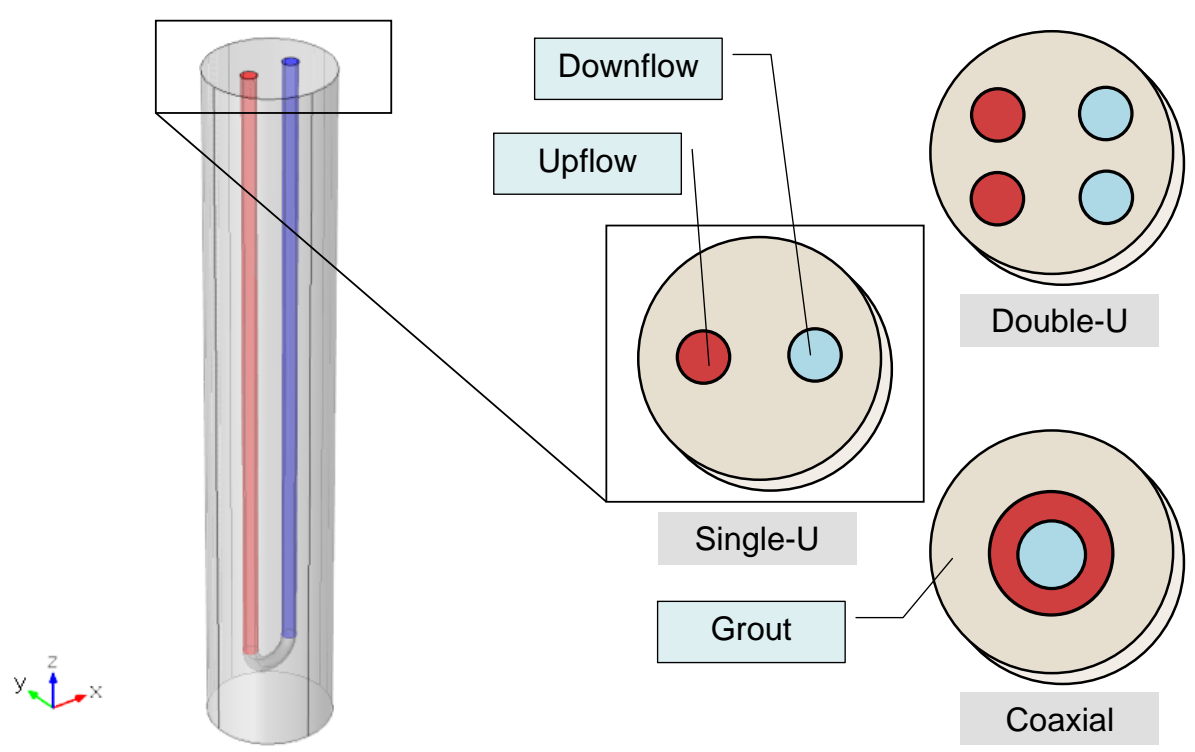

Figure 1.1: Common BHE designs

\subsection{State of Knowledge}

Several literature reviews on the topic of BHEs were published, e.g. by Rawlings and Sykulski [1999], Spitler [2005] or more recently by Javed et al. [2009], Yang et al. [2010] or Rainieri et al. [2011]. In this section, the main progress in borehole heat exchanger modelling up to the current state are briefly described.

\subsubsection{BHE Models}

There are many conceptual approaches to modelling the function of BHEs. The models differ in dimension and complexity; most of them are only valid within a certain range of timescales and under further restrictions and simplifications, as in most cases a representation of all physical processes and parameters occurring inside and around a BHE system is unnecessary. The required level of detail depends on the scope of the numerical study. If short time scales are to be considered, the dynamic behaviour of temperatures and their gradients in the borehole need to be represented, whereas they are irrelevant when the scope of the analysis is concerned with long timescales (several years). For the latter, the temperature spread in the subsurface into the axial direction 
of the borehole becomes significant. Hence, the model region must include the region below the borehole and effects from the upper boundary, i.e. the ground surface.

Due to Ruan and Horton [2010], the different methods of calculating heat transfer in BHEs can be historically divided in three phases: In the 1940's to 1960's, theoretical methods were developed. Between the 1970's and 1980's, the focus was on analytical solutions. Since the late 1980's, along with the remarkable increase of computational capacities, the development of numerical models has become more and more important.

The first approach to modelling a BHE is done by Ingersoll [1954]. The idea is that the borehole is simplified to be a single resistive element around a thermal line source. If the line source is heated at constant rate, Carslaw and Jaeger [1959] provided a simple analytical solution for this problem. The main shortcoming of the line source models is that the simplification of the borehole to an infinite line in an infinite, homogeneous medium does not take into account short-term effects inside the borehole, nor the long time heat transfer in the vertical direction.

Eskilson [1987] carried out pioneering work by creating an axial-radial 2D model and proposed a dimensionless temperature response factor, the g-function. The response of a single BHE that is heated with a unit step heat pulse is represented in this way and analytical superposition is used to capture the interaction between neighbouring boreholes. Since the BHE depth is limited here, long timescales can be considered as vertical heat fluxes are represented. The borehole is simplified to be a single resistive element as well. Eskilson stated that his approach can only be applied at timescales that fulfil the criterion $t>5 r_{b}^{2} / a$ which may amount to at least several hours, but may also lead to times up to a number of days.

For the simulation of BHE systems, shorter time scales in the range of minutes are of interest. The dynamic behaviour of the heat flow inside the borehole and in the nearby ambient is dominated by high horizontal gradients, compared to the low vertical or axial gradients. Yavuzturk and Spitler [1999] reduced the problem to a 2D horizontal cut plane of a borehole to calculate short timescale responses and extend g-function response data. The pipes are represented as pie sector shapes, but the circulating fluid is not explicitly included. Neglecting the thermal mass of the working fluid may lead to short time reaction errors, as its total heat capacity is of the same order as the grouts. Young [2004] improved the accuracy of short time reactions by including the 
fluid thermal mass from the analogy of heat transfer outside of an electrical cable buried in soil. Another recent approach for the numerical representation of the heat capacities is created within the project Geo-Solar-WP $P^{3}$ An adiabatic pipe model with wall capacity is patched before a TRNSYS ${ }^{4}$ BHE type model. The performance of this combined model provides a significantly better short-time performance than the state of the art TRNSYS types (Pärisch et al. [2013]).

2D-horizontal BHE modeling approaches are restricted: No vertical variation can be taken into account. Especially the temperature depth-dependence of the working fluid in the pipes, but also spatial distributions of the subsurface s' thermal parameters and geothermal gradients are neglected if only a horizontal cross section of the system is regarded. Furthermore, simplifying assumptions for the fluid temperatures have to be made. One possibility would be to choose the mean temperature of inlet and outlet for both the down-flow and up-flow branches. Another way is to use the inlet temperature for the down-flow and the outlet temperature for the up-flow branch. In both cases, the assumptions may lead to inaccurate results. The vertical distribution of the pipe fluid temperature can vary strongly and be more complex than the simplifications suggest. However, there are approaches that represent heat transport in all directions by vertical discretisation into several two-dimensional layers with lumped thermal capacities, e.g. Oppelt et al. [2010], or in the form of a network of resistances and capacitances (Bauer [2011]).

Recently, some full 3D approaches came up, e.g. by Li and Zheng [2009] or Rees and He [2013], who present three-dimensional finite volume models, or Signorelli et al. [2007] who analysed thermal BHE responses using a model that is based on the finite-element code FRACTure by Kohl and Hopkirk [1995].

\subsubsection{Thermal Response Tests}

In shallow geothermal applications, (geo-) thermal response tests (GTRTs or TRTs) are the fundamental method to determine the in-situ averaged thermal conductivity $\lambda_{e f f}$ of the subsurface and the borehole thermal resistance $R_{b}$. TRTs are usually performed

\footnotetext{
${ }^{3}$ Hocheffiziente WärmePumpensysteme mit Geothermie- und Solarthermie-Nutzung (high-efficient heat pump systems with geothermal and solar thermal energy sources)

${ }^{4}$ TRNSYS (TRaNsient SYstem Simulation) is a FORTRAN-based commercial software package to simulate the behaviour of transient systems. Its main application is the thermal building simulation, including renewable geothermal or solar-thermal energy sources as modules (types)
} 
when more than one BHE is to be built. In such a case, a pilot drilling is done to gain essential data about the sites geology and a drilling method for the construction of the following BHEs. A TRT in the pilot BHE provides thermal information about the subsurface. This information is important to estimate the required number and the required depths of further drillings. One tries to minimise the overall drilling depth because drilling is, in general, the most expensive part of a shallow geothermal project. Thus, with a reliable estimation of the subsurface thermal conductivity at the project site, an overestimation of the required overall BHE length can be avoided.

The principle of a TRT is to either extract or inject heat at a constant rate and evaluate the time-dependent BHE inlet and outlet temperature development. This provides an indirect measurement of the thermal response of the whole system. The methodologies to handle the TRT data can generally be separated into two main approaches: analytical solutions and numerical models.

Most of the analytical approaches are based on the line source models discussed before. The BHE is approximated as an infinite line source releasing a constant heat flux into the subsurface. An analytical solution for the transient heat conduction problem of infinite line sources is available, e.g. in Carslaw and Jaeger [1959]. Mogensen [1983] presented the first TRT applications with this approach and Eklöf and Gehlin [1996] developed mobile TRT equipment and described the technique and evaluation method in detail. The main restriction of the LSMs (Line Source Models) is that they are only accurate enough after time scales of a certain limit, usually at least one or more days, when a quasi-steady state condition is reached. One approach to reduce the required amount of TRT time is to use a cylinder source approximation (CSM, Cylinder Source Model) instead of a line. Ingersoll [1954], Kavanaugh and Rafferty [1997] and more recently Bernier [2001] made contributions to this topic. Sass and Lehr [2011] showed that the TRT execution time can be reduced by using the cylindrical approach, because the data can already be evaluated in transient state and one does not necessarily have to wait until steady state conditions arrive.

Full numerical TRT evaluation approaches, that do not fall back on analytical heat source approximations, are developed to estimate the unknown thermal properties by inverse calculations, discussed e.g. by Beck et al. [1985]. The deviations of the model results compared to experimental data are minimised by system parameter tuning or 
optimisation, e.g. using the heuristic Nelder-Mead method (Nelder and Mead [1965]). Doing so, it is even possible to distinguish between the short-time and long-time reaction of the model and thus to seperate the effective thermal conductivities between the grout conductivity $\lambda_{g}$ and the soil conductivity $\lambda_{s}$, as e.g. done by Yavuzturk and Spitler [1999] or Bozzoli et al. [2011]. The quality of information that can be gained by inverse modelling depends strongly on the quality of the experimental data. Usually, this data is limited on the BHE in- and outflow temperatures and pipe flow rates. If the experimental data set contains depth-resolved temperature information, even a depth-resolved optimisation is also possible. In such a case, depth-resolved thermal conductivities can be obtained. Pioneering work about this advanced method is done by Fujii et al. [2009].

For an overview about the presented literature references about solutions of the inverse problem of TRT evaluation, some relevant contributions are chronologically summarised in Table 1.1.

\begin{tabular}{lll}
\hline Reference & Approach & inverted parameters \\
\hline Mogensen [1983] & LSM & $R_{b}, \lambda_{s}$ \\
Eskilson [1987] & LSM & $R_{b}, \lambda_{s}$ \\
Kavanaugh and Rafferty [1997] & CSM & $\lambda_{s}, c_{p, s}$ \\
Yavuzturk and Spitler [1999] & 2D num., horiz. slice & $\lambda_{s}, \lambda_{g}$ \\
Austin et al. [2000] & 2D num., horiz. slice & $\lambda_{s}, \lambda_{g}$ \\
Shonder and Beck [2000] & 1D num., horiz. slice, & $\lambda_{s}, \lambda_{g}$ \\
& LSM, CSM & \\
Witte et al. [2002] & 2D num., horiz. slice & $\lambda_{s}, \lambda_{g}$ \\
& LSM & \\
Gehlin and Hellström [2003] & 1D num., & $R_{b}, \lambda_{e f f}$ \\
& LSM, CSM & \\
Signorelli et al. [2007] & 3D num., LSM & $\lambda_{s}$ \\
Fujii et al. [2009] & CSM & $\lambda_{s}(z)$ \\
Bozzoli et al. [2011] & 3D num. & $\lambda_{s}, \lambda_{g}, c_{p, s}, c_{p, g}$ \\
\hline
\end{tabular}

TABLE 1.1: Summary of selected relevant literature contributions about TRT inverse problem solutions, following Rainieri et al. [2011]

\subsection{Motivation}

The share of the authors work within the project Geo-Solar-WP is proposed to develop a numerical model for the simulation of the heat transport phaenomena in shallow geothermal BHEs, which is to be compared with experimental data and state-of-the-art 
approaches (TRNSYS model types). A literature review showed that, within the last decade, a number of approaches have appeared that are somehow limited in various ways and there are not yet many models available that get along with only few simplifying appoximations. Especially until recently, most analytical approaches have overlooked or oversimplified the short-term response of BHEs. However, these responses are an important aspect, as the average heat production intervals often only last for some hours or even less. Within this time scale, the inner BHE thermal masses of the fluid and back-filling material play a major role and must necessarily be looked at. Thus, it was decided to develop and validate a full-3D FEM (Finite Element Method) approach using the commercial software package COMSOL Multiphysics. The intention is to be capable of simulating both short- and long-term responses due to any kind of heat extraction or injection rate. The idea is also to be able to include other processes like advective heat transport due to subsurface flow and to permit any spatial refinement of the material properties involved.

The highly discretised numerical model is appropriate for studies about the impact of geometrical and material properties on the performance of BHEs. Also, the intense activity on the topic of BHE performance and thermal response tests motivated the development of a novel test design: based on the conventional TRT method, the idea is to excite the system with oscillatory signals with varying frequencies, instead of a constant heat step. The intention is to gain additional information about the BHE and the ambient subsurface. 


\section{Chapter 2}

\section{Theoretical Background}

The primarily physical processes treated in this work are heat transport phenomena. The fundamental law that includes all heat transport is the first law of thermodynamics, stating that all closed systems possess an inner energy (U), which can only change if energy is transferred into or out of the system. Since $U$ is a quantity that is difficult to measure and to handle, the basic law is usually rewritten in terms of temperature $(\mathrm{T})$. This leads to the heat equation that appears in different forms and concerns different processes which can change the temperature at a certain point of a system. The heat equation is a partial differential equation (PDE) providing analytical solutions for certain cases, but it needs to be solved numerically in complex situations. The temperature can change due to the presence of heat sources or sinks or by heat transfer. The latter is defined as the movement of energy due to a difference in temperature. There are three mechanisms for heat transfer:

- Conduction Heat conduction is the notation for different heat transfer processes that take place on the molecular scope. Fluid molecules collide and carry impulses through the gas, crystals vibrate and pass vibration to their nearest neighbours (phonons) while metals transfer heat by electrons. These processes have in common that they can be easily described macroscopically because the heat flux is always proportional to the temperature gradient. The proportionality factor is the thermal conductivity $\lambda$.

- Convection Heat convection (or advection) appears when a fluid is in motion and heat is transported by net displacement of the fluid itself. The term convection 
also refers to the heat dissipation from a solid to a fluid, called convective cooling or heating.

- Radiation Radiation of heat takes place by photon emission and absorption. It is a process that plays a role in (semi-) transparent media where photons can propagate. Radiation is therefore not considered in subsurface heat transport processes $^{1}$.

The fundamentals of heat transfer are described in detail by Incropera et al. [2011]. Below, the relevant mechanisms for this work are specified.

\subsection{Heat Transfer in Porous Media}

The heat transport in a porous medium is determined by the partial differential heat transport equation

$$
\left(\rho c_{p}\right)_{e q} \frac{\partial T}{\partial t}+\left(\rho c_{p}\right)_{f} \mathbf{u} \cdot \nabla T=\nabla \cdot\left(\lambda_{e q} \nabla T\right)+Q
$$

This implies that a change in temperature at a point in the porous medium may change due to advective processes (second term, Left Hand Side (LHS)), conduction or by a source/sink (Right Hand Side, RHS). The coefficients for heat transfer, namely volumetric heat capacity and thermal conductivity, are equivalent values for the current ratio of fluid and porous solid. In this macroscopic view approximation, the porous medium is treated as a continuum. The volume fractions of solid and fluid, $\Theta_{s}$ and $\Theta_{f}$, determine the equivalent quantities

$$
\left(\rho c_{p}\right)_{e q}=\Theta_{s} \cdot\left(\rho c_{p}\right)_{s}+\left(1-\Theta_{s}\right) \cdot\left(\rho c_{p}\right)_{f}
$$

and

$$
\lambda_{e q}=\Theta_{s} \lambda_{s}+\left(1-\Theta_{s}\right) \lambda_{f}
$$

with

$$
\Theta_{f}=1-\Theta_{s} .
$$

\footnotetext{
${ }^{1}$ One exception is the subsurface radiation by the sun which leads to daily and seasonal changing heat input to a subsurface heat transfer model. However, this is rather included as a heat source boundary condition and not as a numerically calculated process.
} 


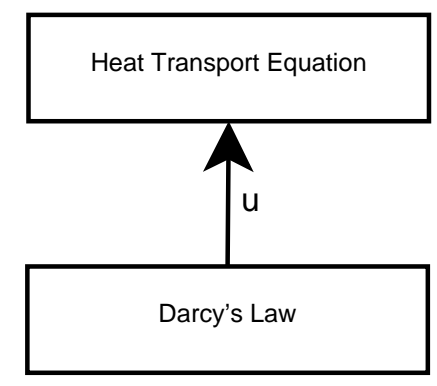

Unidirectional Coupling

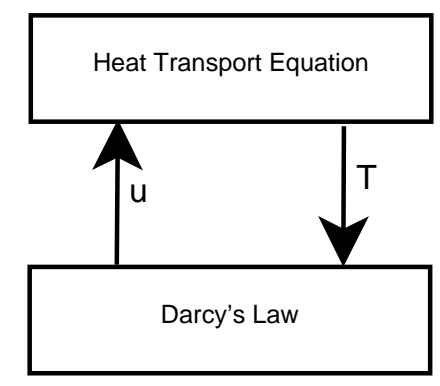

Bidirectional Coupling

Figure 2.1: Example for coupling methods between different PDEs

The velocity field $\mathbf{u}$ in the advective term of Equation 2.1 is gained numerically by solving

$$
-\nabla \cdot K \nabla h=0
$$

which is the combination of Darcy's Law

$$
\mathbf{u}=-\frac{\kappa}{\mu} \nabla p
$$

and the continuity equation for an incompressible fluid

$$
\nabla \cdot \mathbf{u}=0
$$

Note that the parameters of Darcy's law are also temperature dependent. In cases of temperature differences within the regarded model space, the changes of the parameters can become significant and it may be necessary to consider them. The equations then have to be coupled bidirectionally and solved simultaneously.

However, unless indicated otherwise, the groundwater flow is not considered in the numerical models and the advective term in Equation 2.1 vanishes.

\subsection{Heat Transfer in Borehole Heat Exchangers}

A major part of this work deals with the heat transport between BHE pipes and their subsurface ambient. Vertical BHE pipes are the standard shallow geothermal devices. The energy balance of an incompressible fluid flowing in a pipe is described by 


$$
A \rho c_{p} \frac{\partial T}{\partial t}+A \rho c_{p} \mathbf{u} \cdot \nabla T=\nabla \cdot A \lambda_{f} \nabla T+f_{D} \frac{\rho A}{4 r_{i}}|\mathbf{u}|^{3}+Q
$$

The temperature of a fixed point within the pipe may change due to advective heat transport, diffusive processes or internal heat sources / sinks. Within BHE pipes there is no such source or sink, therefore this term vanishes. There is also heat that is generated by friction due to viscous shear, represented by the second term on the RHS, but this effect is negligible in present conditions in common BHE systems and will not be concerned. It should be noticed that the pipe is here regarded as being a three dimensional object and not a one dimensional approximation like in many approaches, for example Al-Khoury et al. [2005], Diersch et al. [2011]. The advantage of this approach is the ability to resolve heat transport processes within and between the pipes, which plays a major role if processes on short time scales and BHE parameters are considered. The heat exchange between the carrier fluid and the grout material finds expression as a Robin Boundary Condition in the PDE system:

$$
\mathbf{n} \cdot(\lambda \nabla T)=\lambda_{r e s} \frac{T_{i n t}-T_{e x t}}{d_{W}}
$$

This means that the radial heat flux is a function of the temperature difference between the two media (borehole and fluid) and of the resulting effective thermal conductivity $\lambda_{\text {res. }}$. The latter is the inverse of the accumulated resistances between the two media. These are the thermal resistance of the pipe wall and the convective heat transfer resistance between the wall and the fluid:

$$
\lambda_{\text {res }}^{-1}=\lambda_{W}^{-1}+\lambda_{\text {fluid }}^{-1}=\lambda_{W}^{-1}+\left[\alpha \cdot r_{i} \cdot \ln \left(\frac{r_{i}+d_{W}}{r_{i}}\right)\right]^{-1}
$$

Hence, the calculation of the heat exchange between fluid and grout requires knowledge of the material parameters and the heat transfer coefficient $\alpha$, which is a variable that depends on the pipe fluid behaviour. 


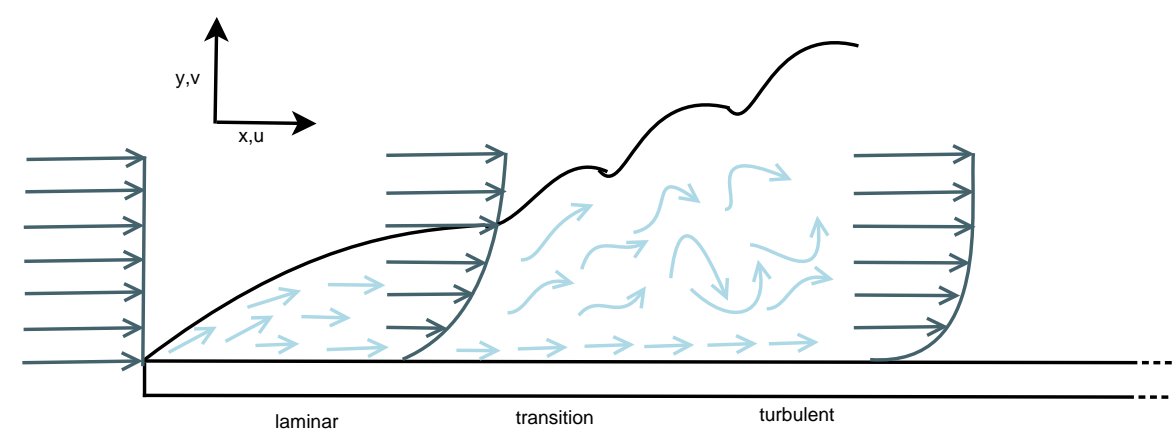

Figure 2.2: Cross-sectional sketch of the velocity field in a pipe for different regimes, modified after Incropera et al. [2011]

\subsubsection{Convective Heat Transfer in Pipes}

The heat exchange of a flowing fluid and a boundary that restricts the flow depends on the dynamic behaviour and circumstances of the fluid motion. The dynamics of a pipe flow are described by the dimensionless Reynolds number ${ }^{2}$.

$$
R e=\frac{2 r_{i} \cdot u}{\nu}
$$

with the hydraulic diameter $2 r_{i}$ as characteristic length, the mean fluid velocity $u$ and kinematic viscosity $\nu$. Re is the relation between specific impulse convection and impulse diffusion in flowing fluids and is used as an indicator for turbulence. Fluid flow in pipes is usually assumed to be laminar at $R e<R e_{\text {crit }} \approx 2040 \pm 10$, see Barkley [2011], and turbulent at $R e>10000$. The range in between, $2040<R e<10000$, is called the transition zone. The transition between laminar and turbulent flow is very complex and the onset and sustainment of turbulence is not completely understood and therefore still a subject of current research interest, e.g Avila et al. [2011].

The presence of turbulence has two major consequences for the BHE: On the one hand, the friction losses along the pipes rise significantly. In laminar flow regimes, the friction losses are proportional to the mean flow velocity while they increase quadratically in turbulent situations. Since these losses have to be compensated for by the fluid pump, the dedicated power demand rises. On the other hand, turbulence also increases the cross-diffusion of heat which leads to diffusion rates that are orders of magnitude higher. The heat transport from the pipe wall to the fluid rises because of the higher temperature

\footnotetext{
${ }^{2}$ After Osborne Reynolds (1842-1912), known for his work on fluid mechanics and turbulence
} 
gradients between wall and fluid caused by the turbulent mixing. The relation between heat transport due to convection and diffusion is the Nusselt number ${ }^{3}$

$$
N u=\frac{2 r \cdot \alpha}{\lambda_{f}}
$$

The knowledge of $N u$ is necessary to calculate the radial heat transfer of pipe flow problems if the flow is not to be calculated explicitly in terms of solving the Navier Stokes equation. There are several empirical approaches to determine $N u$ as a function of the geometry, Re and Prandtl number $\mathrm{Pr}^{4}$

$$
\operatorname{Pr}=\frac{\nu}{a}
$$

the relation between impulse transport due to friction and thermal diffusivity and therefore the link between the temperature and velocity field of a fluid. Due to the principle of similarity, described e.g. by Weber [1930], the three characteristic numbers $R e, N u$, $\operatorname{Pr}$ are sufficient to describe the entire convective heat transfer process. Figure 2.3 shows some of the most common approximations that describe $N u$ as a function of $R e$ in pipes. The formulations are more closely described in Appendix A.

It should be considered that the quantities of the dimensionless numbers 2.11-2.13 are temperature dependent because their arguments are temperature dependent. Figure 2.4 illustrates an example of the significant temperature influence on $R e$ for different flow rates in a Double-U pipe system with water-antifreeze mix of which the temperature dependencies are known and described in Appendix B. The Reynolds number increases with temperature because of the decreasing viscosity. This leads to a shift of the laminarturbulent transition point $(R e \approx 2300)$ which is reached at much lower fluid velocities for high temperatures. Considering the enormous impact of $R e$ on $N u$, as shown in Figure 2.3, it is clear that the influence of the temperature on the pipe flow convection is an important quantity for the heat transfer calculations which is, however, often neglected.

Knowing the Nusselt number allows one to calculate the heat transfer coefficient $\alpha$, which is now the only unknown in Equation 2.12. By inserting $N u$ into Equation 2.10, one

\footnotetext{
${ }^{3}$ Named after Wilhelm Nußelt (1882-1957), developer of the dimensional analysis of heat transfer.

${ }^{4}$ Named after Ludwig Prandtl (1875-1953), founder of the basics of fluid mechanics and the fundamental principles of subsonic aerodynamics and boundary layers
} 


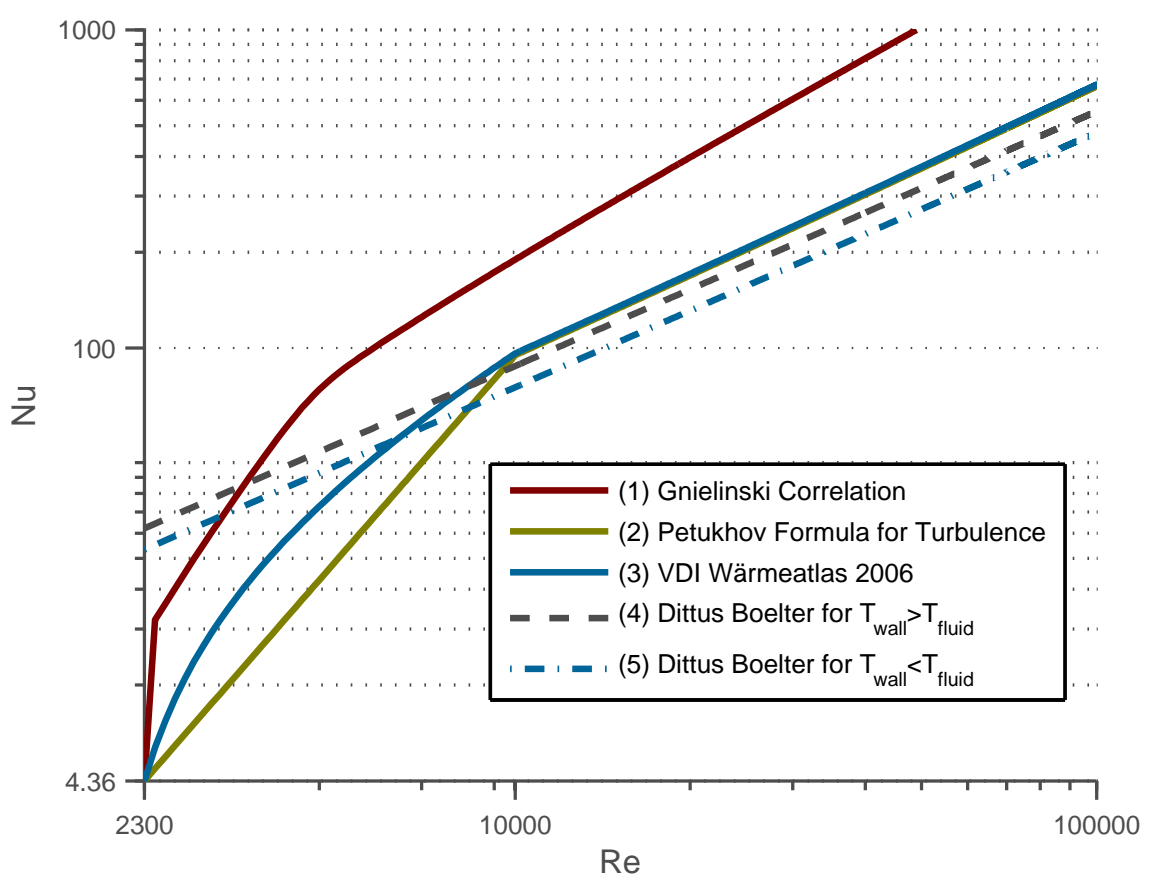

Figure 2.3: Comparison of different pipe flow correlations $N u(R e)$ in the transient regime, $\operatorname{Pr}=9$; (1) Gnielinski [1976], (2) Huber and Schuler [1997], (3) GVC-VDI [2006] (German Engineer Association, Heat Atlas) , (4) + (5) Dittus and Boelter [1930].

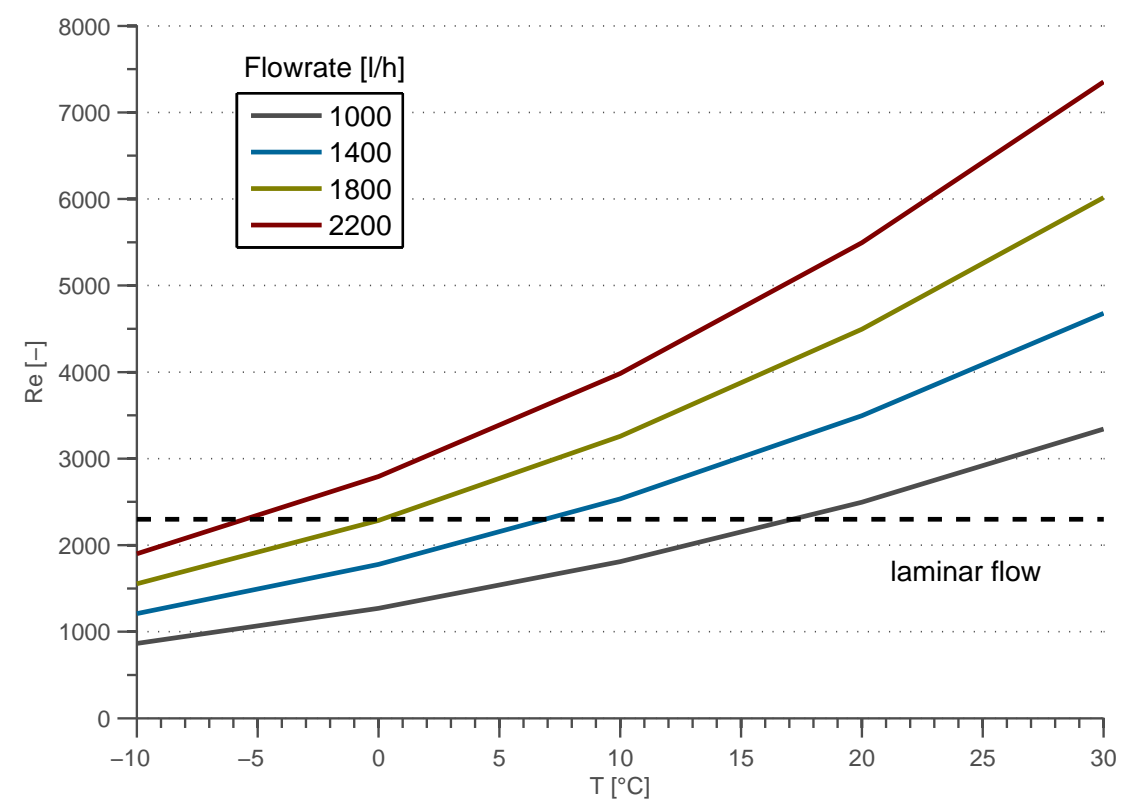

Figure 2.4: Temperature dependence of $R e$ for different flow rates, for the example of a water-antifreeze mix. 


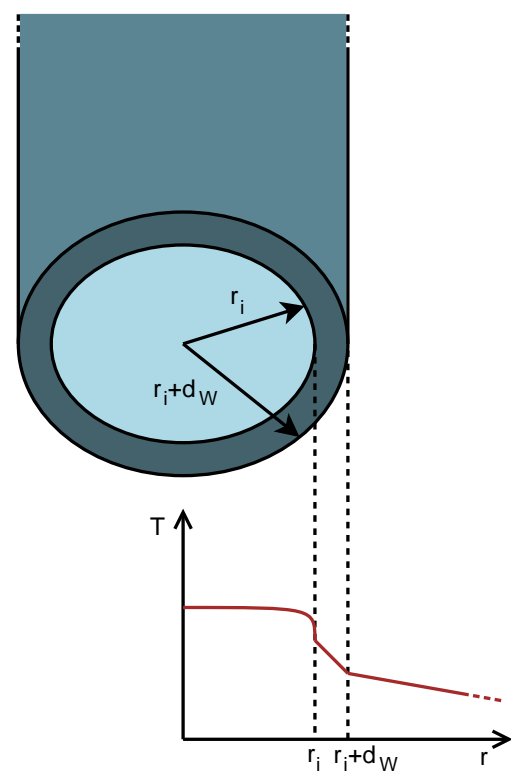

FIGURE 2.5: Temperature profile (qualitative) of a pipe fluid in heat exchange with the subsurface ambiance, here: heat injection

obtains the effective thermal resistance between the pipe fluid and the borehole filling material. In the numerical model, this quantity is included as an internal boundary condition for the pipe flow heat transfer.

Figure 2.5 shows, in a qualitative way, the radial temperature profile that is observed in a BHE pipe during heat injection into the subsurface. The fluid temperature within the pipe is mainly constant due to turbulent mixing of the fluid. Towards the pipe wall, $T$ decreases because heat is transported into the pipe wall, where the temperature further decreases until the subsurface level is reached. $\lambda_{\text {eff }}$ contains and summarises these processes in one quantity.

\section{$2.3 \quad$ Thermal Response Tests}

A temperature change by conductive heat transport in a medium with a constant heat diffusivity $a$ is mathematically described by the heat transport equation

$$
\nabla^{2} T=\frac{\partial^{2} T}{\partial x^{2}}+\frac{\partial^{2} T}{\partial y^{2}}+\frac{\partial^{2} T}{\partial z^{2}}=\frac{1}{a} \frac{\partial T}{\partial t} .
$$


One solution is given by

$$
T(t)=T_{0}+\frac{Q}{8(\pi \lambda t)^{3 / 2}} e^{-\left[\left(x-x^{\prime}\right)^{2}+\left(y-y^{\prime}\right)^{2}+\left(z-z^{\prime}\right)^{2}\right] / 4 a t} .
$$

This expression goes towards $T_{0}$ for all points in space except $\left(x^{\prime}, y^{\prime}, z^{\prime}\right)$, where it goes towards infinity. $T(t)$ can be interpreted as the temperature that appears due to an instantaneously induced amount of heat $Q$ at the point in time $t_{0}$ that distributes diffusively in the medium.

Now assume that a thermal line source is in perfect thermal contact inside the medium which is radially expanded in a way that no temperature changes appear at the radial boundaries $\left(T\left(r_{\text {boundary }}\right)=T_{0}\right)$ and the length of the line be long enough that boundary effects of the ends of the line are negligible. Let the line be heated up by the instantaneous induced heat $Q$. This is comparable to a line of point sources, and at the time $t$ there is

$$
\begin{aligned}
T(t) & =T_{0}+\frac{Q}{8(\pi \lambda t)^{3 / 2}} \int_{-\infty}^{\infty} d z^{\prime} e^{-\left[\left(x-x^{\prime}\right)^{2}+\left(y-y^{\prime}\right)^{2}+\left(z-z^{\prime}\right)^{2}\right] / 4 a t} \\
& =T_{0}+\frac{Q}{4 \pi \lambda t} e^{-r^{2} / 4 a t}
\end{aligned}
$$

where $r^{2}=\left(x-x^{\prime}\right)^{2}+\left(y-y^{\prime}\right)^{2}$.

This is the exact solution of a heat pulse (delta distribution) which is not feasible in practice. A viable realisation is a continuous and equally distributed heat injection $q(t)$ per unit length, starting at $t=0$; The solution then becomes

$$
T(t)=T_{0}+\frac{1}{4 \pi \lambda} \int_{0}^{t} \frac{q\left(t^{\prime}\right)}{t-t^{\prime}} e^{-r^{2} / 4 a\left(t-t^{\prime}\right)} d t^{\prime} .
$$

In case of a temporary constant heating rate $q(t>0)=q_{0}$ and after substitution, this expression becomes

$$
T(t)=T_{0}+\frac{q_{0}}{4 \pi \lambda} \int_{r^{2} / 4 \pi a}^{\infty} \frac{e^{-u}}{u} d u .
$$

It is proven that there is no elementary solution for the appearing exponential integral

$$
-E i(-x)=\int_{x}^{\infty} \frac{e^{-u}}{u} d u .
$$


Nevertheless, it can be approximated by a series expansion, see e.g. Abramowitz and Stegun [1964]. For small arguments x, Equation 2.20 can be simplified to

$$
E i(-x)=\gamma+\ln x-x+\frac{1}{4} x^{2}+\mathcal{O}\left(x^{3}\right) .
$$

This corresponds to a large time approximation of the temperature problem of a BHE. Considering only the first two terms, the approximate solution of the temperature at the borehole wall with radius $r_{b}$ is

$$
T_{b}(t)=T_{0}+\frac{q_{0}}{4 \pi \lambda}\left[\ln \left(4 a t / r_{b}^{2}\right)-\gamma\right]
$$

The maximum error of this approximation is only 2 percent compared to a full series expansion, when the condition $t \geq \frac{5 r_{b}^{2}}{a}$ is fulfilled. This solution is of great importance for the evaluation of a thermal response test. It follows directly that the overall thermal conductivity of the whole system can be easily derived from the slope of the temperature in a semi-logarithmic plot of Equation 2.22 after waiting long enough until the slope is constant:

$$
\lambda_{e f f}=\frac{q_{0}}{4 \pi} \cdot\left(\frac{\Delta T_{b}}{\Delta \log (t)}\right)^{-1}
$$

The borehole wall temperature $T_{b}$ is usually not measurable; only the (mean) fluid temperature $T_{f}$ can be experimentally determined as the arithmetic mean between the in- and outflow temperatures. The thermal resistance $R_{b}$ between the fluid and the borehole wall

$$
T_{f}-T_{b}=R_{b} \cdot q
$$

connects these two quantities.

The reduction of a BHE to a line source is only valid in the range of a number of approximations and it has to be kept in mind that there are some experimental restrictions. The most important points that have to be considered are outlined here:

1. Constant heat injection rate: The solution of the line source approximation (Equation 2.24) is based on a step- or Heaviside-function of the heat injection rate. Deviations of the injection rate lead to errors in the interpretation of the temperature development. In practice, the heat injection rate is never really constant and the error caused by this must be considered. 
2. Homogeneous initial temperature: $T_{0}$ is assumed to be constant over the whole regarded domain. This is not true in reality; there are different possible reasons for deviations of the undisturbed ground temperature. The upper 10-15m are dominated by the temperature above the subsurface. In European latitudes, this may vary in the range of some degrees Celsius. After all, this may be a significant influence especially in very shallow BHEs. Furthermore, one finds a geothermal gradient $\Gamma=d T / d z \approx 0.03\left[K^{-1}\right]$, which leads to a depth-dependent temperature distribution and which can be even higher due to local anomalies. Eskilson [1987] showed that one can use the temperature in the middle of the BHE depth to reduce the error of the geothermal gradient to less than $1 \%$.

3. Thermal parameter distribution: It is assumed that the subsurface heat transport parameters are homogeneous and constant. In general, this is not the case at any geological site. Different geological structures feature different inherent thermal properties (thermal conductivity, density, heat capacity). In addition, the effective values of the named properties are functions of the porosity (represented in Equation 2.2 and Equation 2.3), if the BHE is located in an aquifer, and even depending on the saturation in the unsaturated zones. Both, porosity and saturation, are in general not constant all over the vertical elongation of a BHE. Thus, the integral experimental result of a TRT, which includes all these inhomogeneities, may lead to erroneous assessments for the required depth of the borehole, for example if the thermal conductivity is significantly higher in lower formations ${ }^{5}$. A recently developed depth-orientated method (Fujii et al. [2009]) uses depth-resolved temperature data from optical fibre sensors for depth-resolved TRT evaluation. Only with such a technique is it possible to consider axial variations of the borehole's ambient parameters.

4. Temperature dependence of inherent parameters: All involved parameters are, more or less, functions of the temperature. This is completely neglected in standard TRT evaluations, but it is also neglected in most numerical simulation approaches because it is computationally expensive to add this bi-directional

\footnotetext{
${ }^{5}$ Signorelli et al. [2007] showed numerically that a heterogeneous subsurface thermal conductivity has an influence on the resulting $\lambda_{e f f}$. Therefore, a homogeneous model is compared with one that is vertically divided in two domains with different values but the same mean value. The evaluation of TRTs lead to a lower $\lambda_{e} f f$ in the heterogeneous case
} 
coupling to the governing equations. The most frequently used argument for neglecting this is that the temperature variations in BHEs are supposed to be low, in the range of only some degrees Celsius, and so are the parameter changes. The real influence of this approximation, especially regarding the short-time scale, is discussed in Section 4.1.1.

5. Conductive heat transport: The line source approximation is a solution of the conductive heat transport equation. Advective heat transport may appear in different ways. Subsurface flow due to hydraulic gradients or precipitation events may increase the effective thermal conductivity significantly. The consequences are treated in the results chapter or in detail e.g. by Gehlin and Hellström [2003], Huber [2013], Kölbel [2010]. It is also possible that the temperature disturbances in the porous medium caused by the TRT lead to local convection in the ambient of the BHE, as discussed by Bringedal et al. [2013]. If the borehole is not backfilled but rather left open and groundwater filled, as is common practice in Swedish hard rock, convection may also occur inside the borehole, leading to a higher heat exchange rate and therefore a lower thermal resistance of the BHE. This topic is discussed in detail by Gustafsson [2006]. 


\section{Chapter 3}

\section{Methods}

The main focus of the thesis is numerical simulations. The numerical models are partially based on the background of an experimental test site, which is part of the EFRE project Geo-Solar-WP. In this chapter, the information and issues from the test site that are relevant to the numerical model are presented. After that, the numerical implementation of the borehole heat exchanger model is introduced.

\subsection{Experimental Test Site}

The experimental BHE test site referred to in this work is located at the Institut für Solarenergieforschung Hameln (ISFH, Institute for Solar Energy Research Hamelin). It is part of an extensive test facility, consisting of two different heat pumps and three programmable high precision modules that can be used to emulate solar collector circuits, domestic hot water and space heating circuits. The facility was built as part of the EFRE Project Geo-Solar-WP (High-efficient heat pump systems with geothermal and solar thermal energy sources), see also ISFH [2010]. It allows one to investigate the different solar and ground-coupled heat pump combinations that are the main subjects of the project. Pärisch et al. [2011] give a more detailed description of the facility and its components.

The subsurface part of the experimental site consists of three BHEs in triangular arrangement (SW, SN, SO) and two groundwater wells (BM, BS) for the performance of hydro-geological tests in different depths. Figure 3.1 shows a map of this test site. 


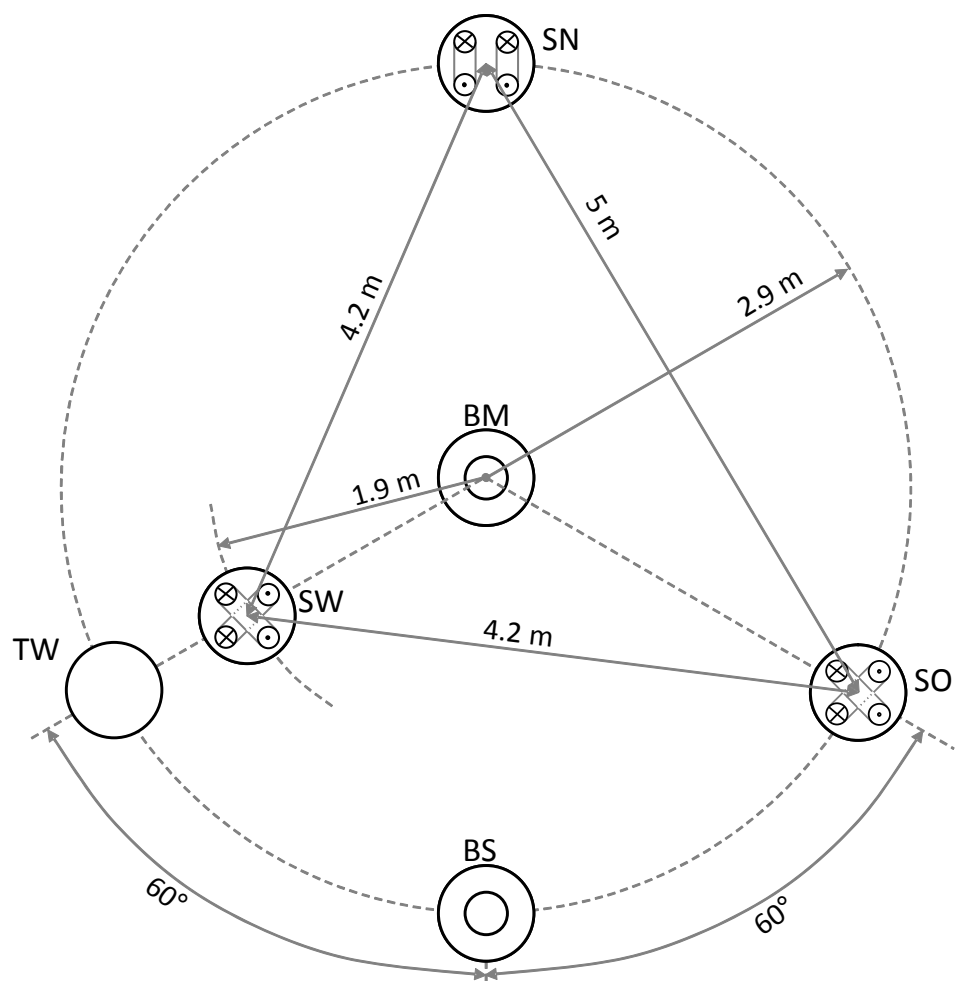

Figure 3.1: Map of the ISFH test field, from Pärisch et al. [2011]. The BHE drillings SN (north), SO (east) and SW (west) are located in a triangular arrangement. TW denotes a temperature measurement borehole, BM and BS are pumping wells with hydraulic contact to the groundwater.

Originally, the three BHEs were planned to be arranged as an equilateral triangle, but the western BHE (TW) was choked and could thus not be used further. SW was drilled to replace the defective BHE and the drilling spot was moved one metre towards the centre of the triangle.

There is one additional BHE (SX) in north-western direction, outside of the triangle and not represented in the Figure. SX was first used for preliminary tests but deactivated later because it did not match the requirements of the project planning; it came up with a relatively high horizontal deviation. The low horizontal deviations of the later drillings (as seen in Table 3.1) were achieved by drilling with double-head rotary equipment down to the final depth. The maximal deviation of only $0.89[\mathrm{~m}]$ at the southern well (BS) is still small enough to regard the low deviation effort as a success and the boreholes as vertical.

All heat exchanger boreholes at the test site are equipped with Double-U BHEs. The pipes in SW and SN are arranged parallel to each other, while those in SO are connected crosswise. To provide the distances between the pipes inside the boreholes, spacers are 


\begin{tabular}{llrrr}
\hline Name & Type & $\begin{array}{r}\text { Drilling } \\
\text { depth }[\mathrm{m}]\end{array}$ & $\begin{array}{r}\text { Eff. depth } \\
{[\mathrm{m}]}\end{array}$ & $\begin{array}{r}\text { Max. horizontal } \\
\text { deviation }[\mathrm{m}]\end{array}$ \\
\hline SN & BHE & 71 & 68.5 & 0.27 \\
SO & BHE & 71 & 69.3 & 0.38 \\
SW & BHE & 71 & 69.5 & 0.15 \\
BM & Well & 67 & 67 & 0.53 \\
BS & Well & 70 & 67 & 0.89 \\
TW & Temp. & 70 & 68.8 & 0.53 \\
\hline
\end{tabular}

TABle 3.1: Parameters and thermal equipment of the drillings, after Pärisch et al. [2011].

installed about every one metre. The pipes and the measurement technique cables are conducted through a horizontal chute at a depth of $1.5[\mathrm{~m}]$, leading to the testfacility. Inside the chute, there are also spacers between the pipes and all of the pipes are thermally isolated there.

Every borehole is equipped with at least one distributed temperature system (DTS), which is a technology based on the temperature dependence of Raman scattering in fibre optic cables and run time differences of optical signal reflections. Additionally, the BHEs are provided with temperature measurement chains, consisting of 10 equally distributed Pt100 sensors. This well-equipped instrumentation was installed to allow detailed monitoring of subsurface heat transfer processes.

The geological layer series of the subsurface is briefly illustrated in Figure 3.2. The layers can be divided into two main sections: the first 13 metres are sandstone, underlain by a mixture of clay-stone and marl-stone in slightly varying composition. The groundwater table is at a depth of about $28[\mathrm{~m}]$.

In a preliminary TRT that was performed in SX, the subsurface effective thermal conductivity at the test site was determined to be $\lambda_{\text {eff }}=2.30\left[\mathrm{Wm}^{-1} \mathrm{~K}^{-1}\right]$ by an external company (geoENERGIE Konzept GmbH). Results from TRTs at the other BHEs that were done during the Geo-Solar-WP project are summed up in Table 3.2.

The deviation of the $R_{b}$ values between the three BHEs is noticeable.All three BHEs are back-filled with the same grout material, but the filling methods are different. This may cause the significant differences between the thermal borehole resistance values $R_{b}$. Also, the resulting effective thermal conductivities differ, although the maximum lateral distance of the boreholes is only about 5 metres. 


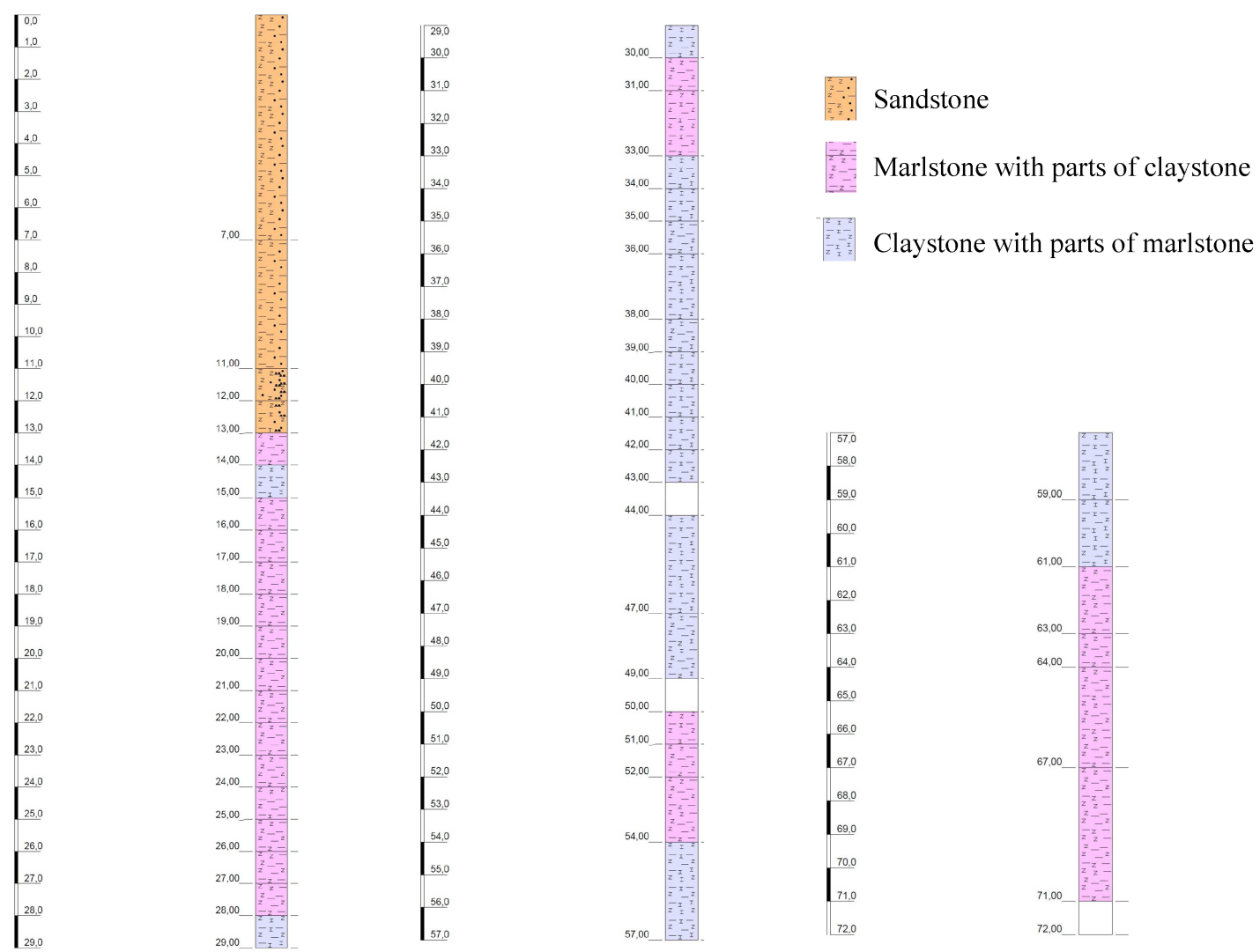

FIGURE 3.2: Subsurface layers at the ISFH test site from Pärisch et al. [2011], modeled after LBEG.

\begin{tabular}{ccc}
\hline BHE & $\lambda_{\text {eff }}\left[W m^{-1} K^{-1}\right]$ & $R_{b}\left[m K W^{-1}\right]$ \\
\hline SN & 2.25 & 0.117 \\
SO & 2.61 & 0.100 \\
SW & 2.29 & 0.071 \\
\hline
\end{tabular}

TABLE 3.2: Experimental TRT results from the ISFH test site (Voss [2011])

\subsection{Numerical Implementation}

The relevant physical processes in a BHE discussed above and its ambient subsurface region are implemented in numerical models, using the commercial software package COMSOL Multiphysics ${ }^{1}$. The purposes of the models are accurate representations of the heat transport phenomena in the pipes, the borehole and the subsurface. However, reasonable simplifications of some physical processes must have been carried out to reduce the complexity of the problem.

\footnotetext{
${ }^{1}$ The COMSOL software versions used for this work are: 4.0, 4.0a, 4.1, 4.2, 4.2a, 4.3, 4.3a, 4.3b, 4.4
} 


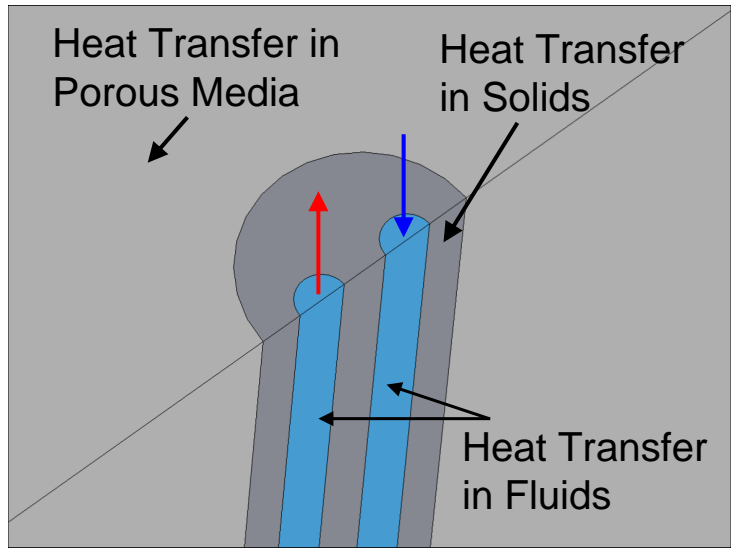

FIGURE 3.3: Different domains of the 3D Single-U pipe model

The models consist of different geometric domains (pipes, borehole and subsurface), and in each domain a particular physical equation is defined. Figure 3.3 shows the domains and the equations that are solved within the domains, for the 3D Double-U pipe case.

The subsurface is considered to be a porous medium, the heat transport is calculated by solving Equation 2.1 numerically. The subsurface parameters used in the model are temperature-independent constant values due to the lack of information about the thermal dependencies. However, the temperature-dependence of the water fraction of the subsurface is well known and taken into account in the models. Unless otherwise indicated, the advective term of the heat transport equation vanishes because subsurface flow is not considered.

The geometric dimension of the domain must always fulfil the condition that the horizontal outer boundary is far away enough from the BHE, so that the heat transport processes are not disturbed by boundary effects. The form of the subsurface is cylindrical, resulting in a constant distance from the outer boundaries. The compliance of the condition is verified after each computation and the domain is re-sized if the criterion is not fulfilled. When time scales below a year or so are considered, the vertical heat flow in the subsurface is negligible (discussed in Section 1.2.1). Thus, it is sufficient to have the same depth for the subsurface as for the BHEs.

Heat transport in the borehole domain is also represented by the heat transport equation, but without advective term and only one domain material, the grout filling. The standard values of the grout properties are then taken from the back-filling (HeidelbergCement) at the ISFH test site. By default, the borehole and the subsurface are 
thermally perfectly connected, but if required a thin thermally resistive layer between these two domains can be added.

The geometry of the borehole is straight vertical with a cylindrical shape and so are the heat pipes within the borehole. Within the pipes, the heat transport is represented in a similar way to the outer domains; the heat balance equation is solved and includes the advective term. However, the velocity field $\mathbf{u}$ of the advective term is not explicitly solved. Instead, the working fluid velocity in the pipes is set to a constant value in z-direction:

$$
\mathbf{u}:=u_{0} \cdot \mathbf{e}_{z}=\frac{1}{2} \cdot \frac{\dot{V}}{\pi r_{i}^{2}} \cdot \mathbf{e}_{z}
$$

Hence, the pipe velocity is a function of the flow-rate $\dot{V}$ and is zero in $\mathrm{x}$ - and y-direction. This formulation is, of course, physically incorrect. The real velocity field of a pipe flow differs from this idealisation: The velocity vanishes at the walls due to friction, leading to a no-slip boundary condition $\left(u_{x}=u_{y}=u_{z}=0\right)$. This condition leads to velocity profiles that depend on the Reynolds number, as shown in Figure 2.2. The no-slip condition leads to a higher thermal resistance between the walls and the fluid because it interferes with the advective transport. However, the numerical effort to solve the pipe flow problem in a BHE physically exact would outreach the current computational possibilities. Also, the correct fluid velocity field is not necessarily needed: the heat transport between the pipe walls and the fluid is calculated using the correlation functions from Section 2.2.1. The resulting thermal resistance is applied to the model as a thin thermally resistive layer with the resistance due to Equation 2.10. The turbulent mixing of the pipe fluid is approximated by a very high thermal conductivity in radial direction:

$$
\lambda_{\text {fluid }, x, y}:=100 \cdot \lambda_{\text {fluid }}
$$

At the bottom of the BHE, the down-flow and up-flow branches are connected. This is numerically realised by calculating the mean temperature $\bar{T}_{\text {down,bottom }}$ at the bottom of the down-flow pipe and assigning it as a boundary constraint of the up-flow pipe: 


$$
T_{\text {up }, \text { bottom }}:=\frac{\int_{A} T_{\text {down }, \text { bottom }} d A}{\pi r_{i}^{2}}
$$

Please note that this approximation does not take into account the thermal processes at the turning point at the bottom of a BHE. A numerical representation of that part would be possible, e.g. by creating a U-shaped connection piece between the pipes. However, this part of the BHE is neglected because a negligible influence on the BHE system is assumed and the benefit of a numerical representation would not justify the effort.

The injection or extraction of heat at a BHE is performed in two ways: the numerically easiest possibility is to define the inflow temperature $T_{i n}$ of the working fluid (Dirichlet condition at the inflow boundary). In common applications, a BHE is rather operated with a heat pump which demands a certain thermal power $P$ from the BHE. This amount of heat leads to a certain temperature difference between the in- and outlets of the BHE:

$$
P_{0}=\left(T_{\text {in }}-T_{\text {out }}\right) \cdot \dot{V} \cdot \rho_{f} \cdot c_{p, f}
$$

It follows as Dirichlet boundary condition for the inflow temperature

$$
T_{\text {in }}=T_{\text {out }}+\frac{P_{0}}{\dot{V} \cdot \rho_{f} \cdot c_{p, f}} .
$$

The sign of $Q_{0}$ is positive in the case of heat injection into the subsurface because in this case the temperature at the $\mathrm{BHE}$ inlet is higher than at the outlet. $Q_{0}$ is negative in case of heat extraction, respectively. In the model, the outflow temperature is determined analog to the bottom temperatures by numerical integration over the whole pipe cross section.

The temperature inlet boundary condition depends on the outlet temperature and the flow-rate. Besides that, the fluid properties $\rho_{f}$ and $c_{p, f}$ are not constants, but rather temperature-dependent variables with known functional correlations (Appendix B). This is considered in the numerical model. The influence of the correct representation of the variable fluid parameters is discussed below in the results chapter. 


\subsubsection{Mesh Verification}

The models of the BHEs and their subsurface ambient are discretised using numerically finite element meshes. Although the program used (COMSOL Multiphysics) provides reasonable working meshing algorithms, the mesh is generated in a semi-automatic way due to the extreme length-radius ratio of the pipes inside the boreholes. The automated routine would create a mesh of free tetrahedrals. Since all geometrical properties have to be considered, the tetrahedrals would need to be very small inside of the pipes and slowly increasing towards the outer areas of the model. In this case, the resolution in z-direction would be finer as needed because the temperature gradients in z-direction are estimated to be much lower than in radial direction. Thus, the mesh is created by discretising the top plane of the cylindrical geometry with a free triangle distribution. The meshed plane is then swept down to the bottom of the model. The resulting mesh elements are prisms.

According to this method, the resolution of the mesh is controlled by two parameters: The number of grid points in the $x-y$-plane (radial resolution, see Figure 3.4) and the number of planes that are swept down to the bottom (vertical resolution).
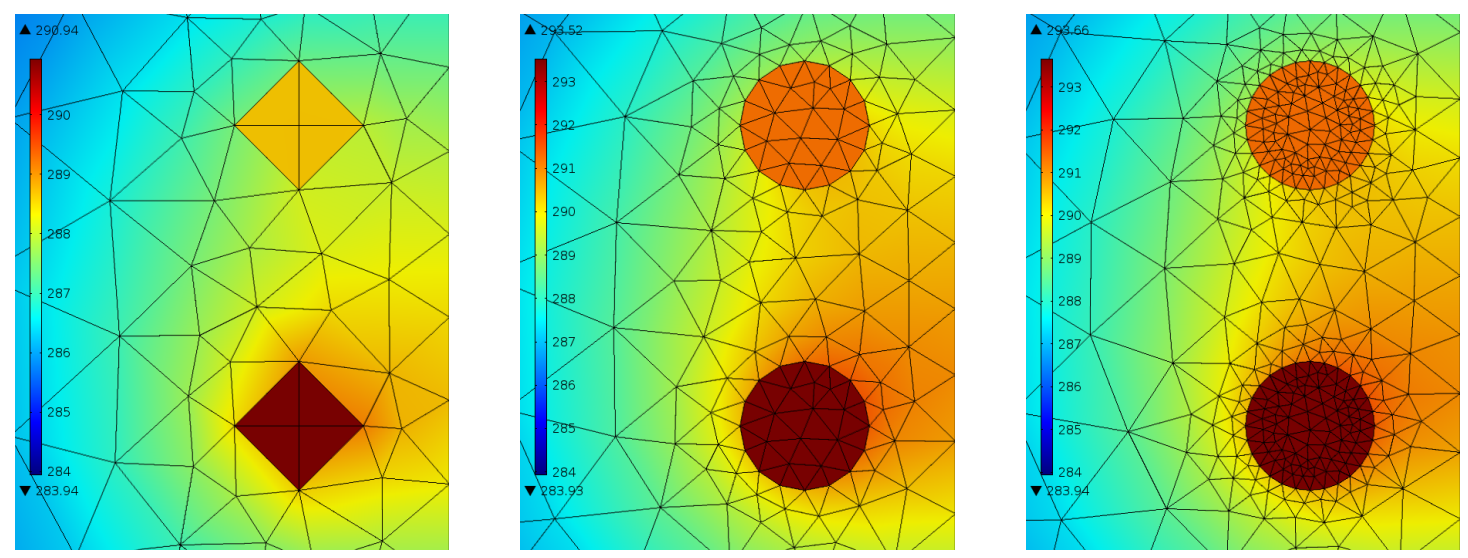

FiguRE 3.4: Solution and grid visualization for different radial resolutions (very coarse to very fine) of the Double-U geometry. The right boundaries of the images are the symmetry planes of the models, only one half of the model geometry has to be considered.

The reasonable choice of these parameters is a compromise between acceptable computation time and accuracy. In Figure 3.5, it is shown how the computation time increases with the increasing number of degrees of freedom. The results are obtained from a benchmark model that was set up on the basis of the geometric properties and the thermal parameters of the ISFH test site BHEs. 

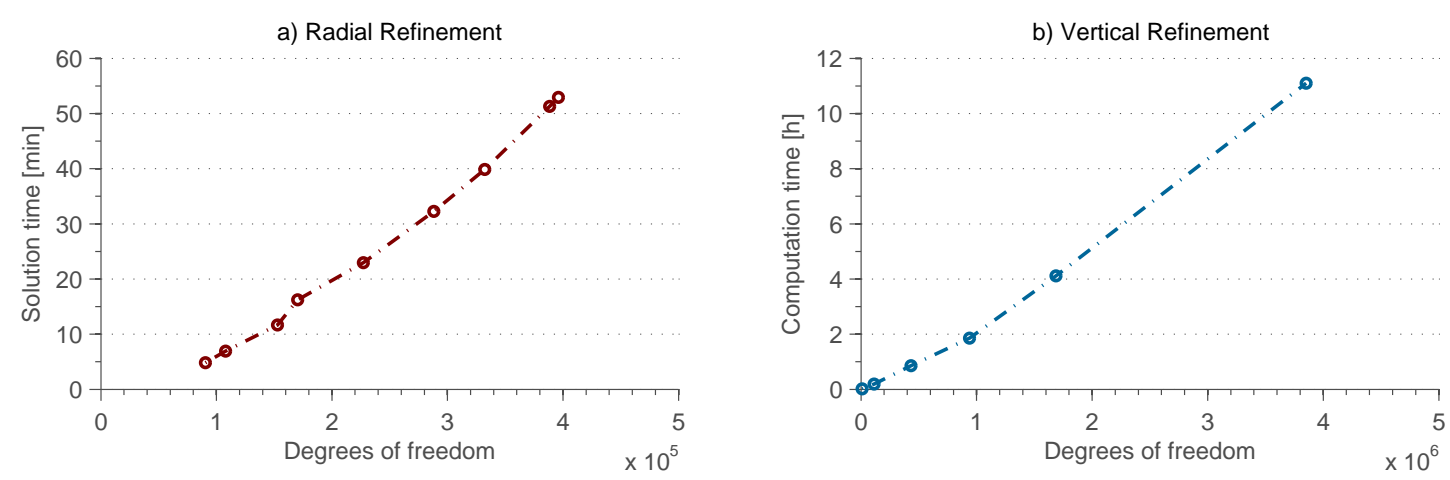

FIGURE 3.5: Increase of the computation time in dependence of the number of degrees of freedom for a $5[\mathrm{~h}]$-heat-injection Double-U simulation. The mesh refinement is executed in radial (a) and vertical (b) direction.
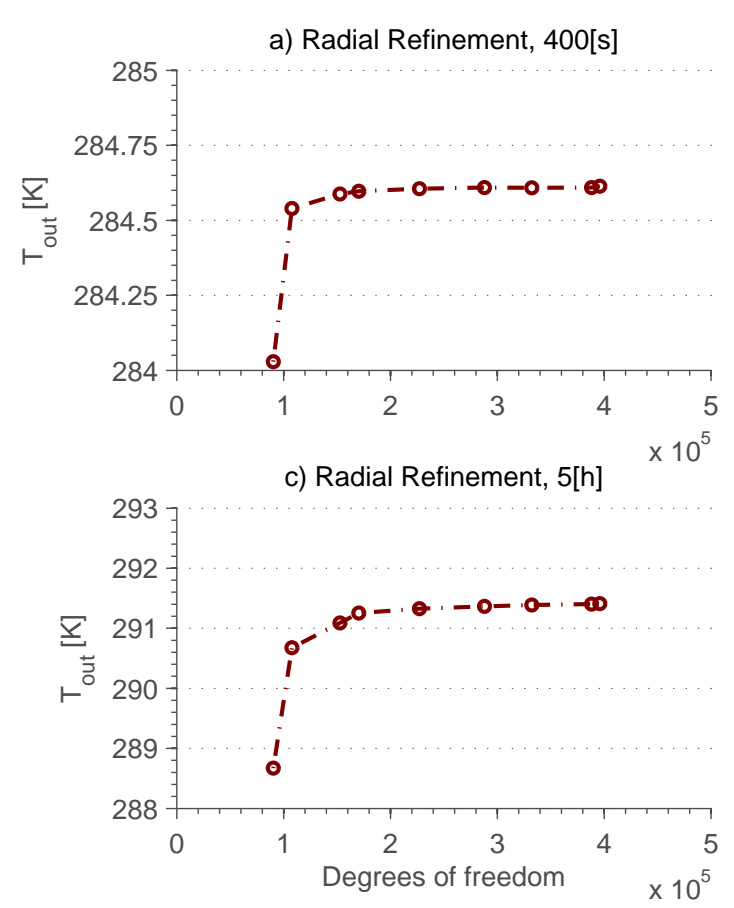

b) Vertical Refinement, 400[s]

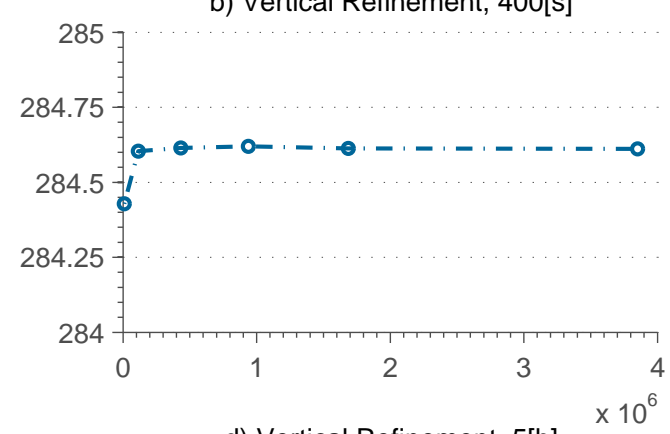

d) Vertical Refinement, 5[h]

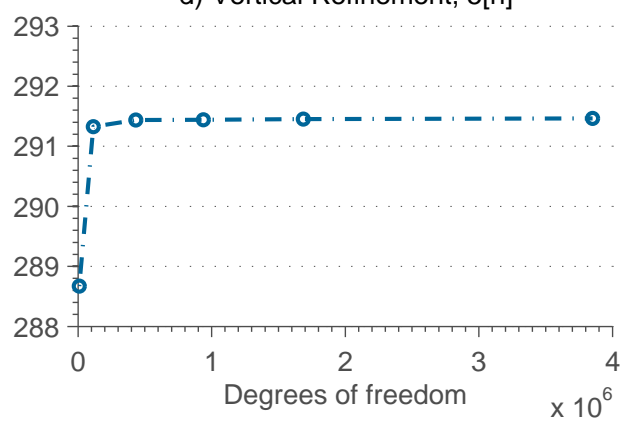

Figure 3.6: Influence of the mesh size on the outflow temperature at a constant heat injection Double-U simulation after $400[\mathrm{~s}](\mathrm{a}, \mathrm{b})$ and $5[\mathrm{~h}](\mathrm{c}, \mathrm{d})$. The mesh refinement is executed in radial $(\mathrm{a}, \mathrm{c})$ and vertical $(\mathrm{b}, \mathrm{d})$ direction.

The accuracy of the model does not increase in the same way as the computation time, it rather reaches a saturation plateau for both cases, radial (Figure 3.6, a) and c)) and vertical (Figure 3.6, b) and d)) refinement. The plateau is reached at about 180.000 degrees of freedom for radial refinement and a comparable value for vertical refinement. The meshes that are used in the further investigations are created due to the experiences of the mesh study results. 


\section{Chapter 4}

\section{Results}

In this chapter, the results from the numerical models are presented. First the validations are shown, and the second part is about the results of parametric studies that are performed with the models.

\subsection{Model Validations}

The model verifications and validations are processes that provide evidence of the correctness and/or accuracy of computational results (Oberkampf and Roy [2010]). Verification can simply be described as solving the equations right. This aspect is discussed in Section 3.2. The validation (solving the right equations) are treated below: results from the numerical models are presented and compared to reference models found in the literature in Section 4.1.1. In Section 4.1.2, the models are also compared to very accurate experimental reference values to prove the reliability of the numerical concept.

\subsubsection{Type Curves}

Results of test runs of the numerical model introduced in this work are presented. Therefore, all necessary parameters in the equations are selected as typical values for BHE systems and stationary and transient model runs are performed. The results are characteristic type curves that can be compared to other numerical models, e.g. Diersch et al. 
[2010], Rees and He [2013], Wołoszyn and Gołaś [2013] or experimental data, e.g. Acuña [2010] from the literature.

\section{Long Timescale}

The thermal response of a BHE at long timescales is studied by adding constant boundary conditions to the model and calculating the stationary solution. For this reason, the temperature far field boundary conditions, i.e. the outer boundaries of the cylindrical subsurface domain, are set to Dirichlet conditions $\left(T_{R}=\right.$ const.) to provide a non-zero stationary solution. The inflow boundary condition is set to be $10[K]$ higher than the far field value, which leads to heat flow from the pipes into the borehole and subsurface. A parametric study is performed with different flow rates. The flow rates vary from 0.1 $[\mathrm{m} / \mathrm{s}]$ to $0.5[\mathrm{~m} / \mathrm{s}]$, whereby the highest value is typical for standard applications. The whole model adjustment is comparable to the study of Rees and He [2013].

The depth-resolved temperature distributions of the parametric study are shown in Figure 4.1. The temperature trend is linear and uniformly distributed over both downflow and up-flow branches at high velocities. This matches to the assumption that is made to determine the mean pipe fluid temperature - it is usually approximated to be the arithmetic mean temperature between in- and outflow of the $\mathrm{BHE}^{1}$. However, this approximation becomes inaccurate when the flow rate is decreased. The slope is clearly no longer linear and the temperature spread between the legs is much larger and asymmetrically shifted to the left (lower temperatures) at $\mathrm{u}=0.1[\mathrm{~m} / \mathrm{s}]$. The reason for this is that the residence time of the fluid in the pipes increases and it has therefore more time to cool down. The greatest temperature spread between the branches comes up at the top of the BHE, where the maximal temperature of the inlet meets the cooled down branch of the outlet. Thus, the inter-pipe heat transfer, or thermal shortcut, is maximised there as seen in Figure 4.2. Although the inter-pipe heat transfer is maximal at low velocities, the borehole-subsurface heat transfer is increased at higher velocities. This is not only due to the fact that the heat injection rate is proportional to the flow rate (when a constant temperature, but also because of the increased turbulence level in the pipes and the resulting higher heat transfer coefficient. Figure 4.3 shows that the borehole-subsurface heat transfer is also more equally distributed at higher

\footnotetext{
${ }^{1}$ Sass and Lehr [2011] propose to utilise the logarithmic mean temperature instead because of the radial symmetry of the problem
} 


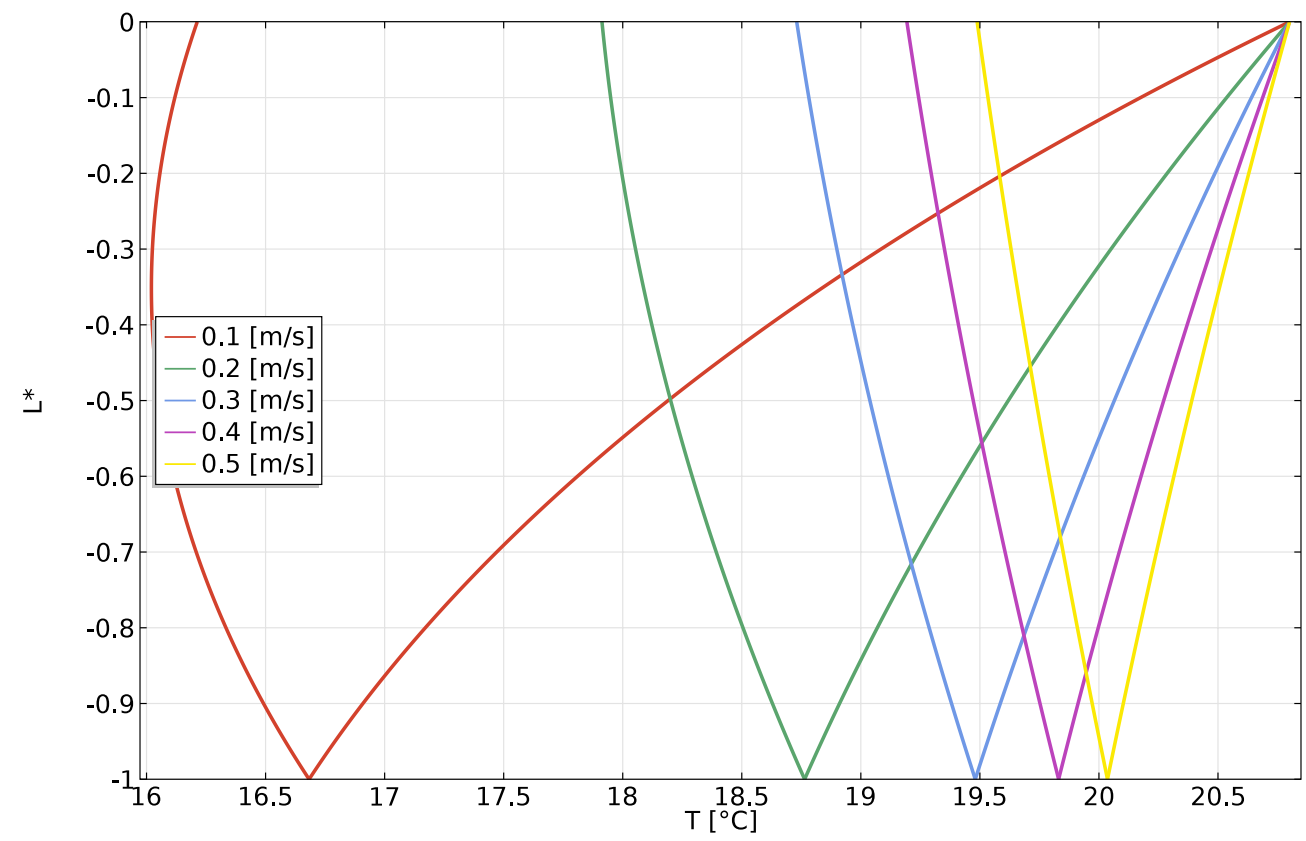

FiguRE 4.1: Fluid temperatures for down- and up-flow branches at different flow rates in the Double-U BHE model

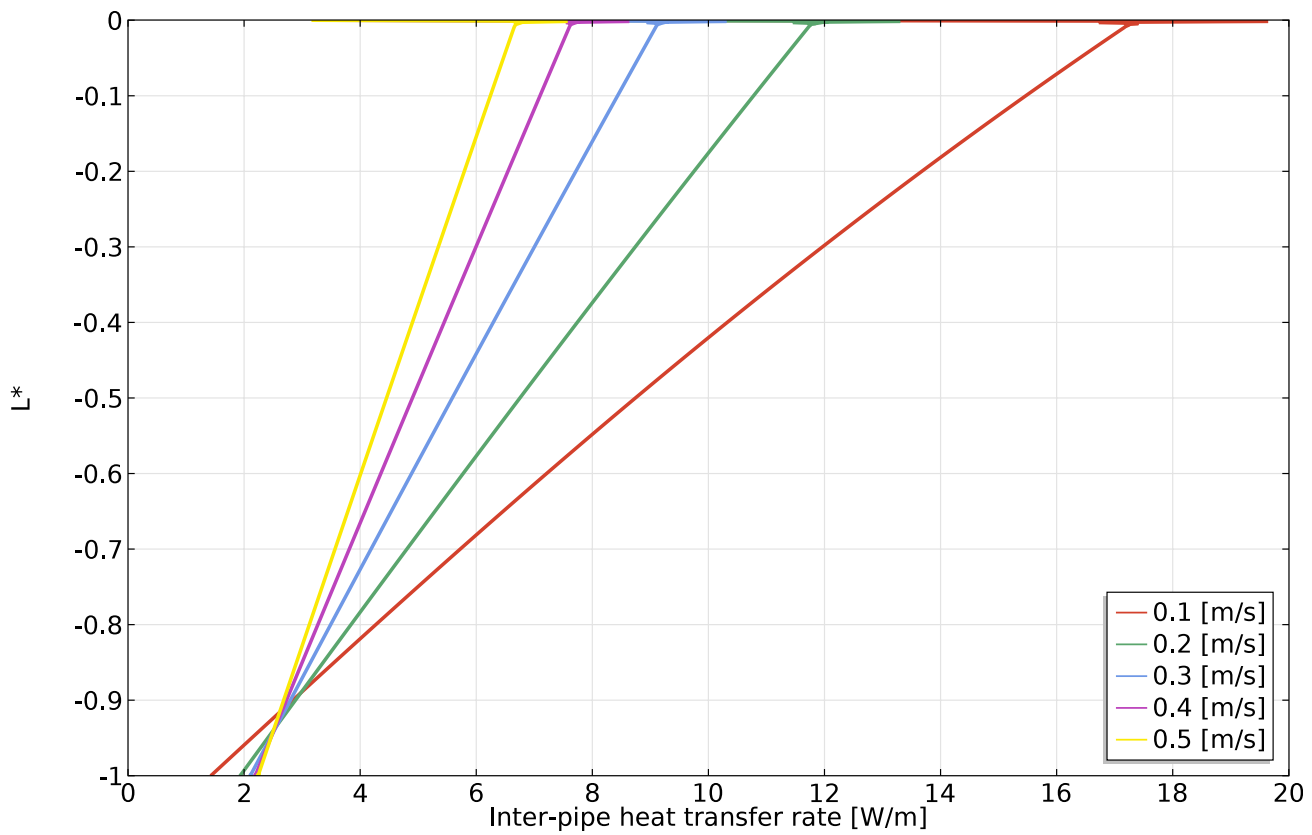

FiguRE 4.2: Heat transfer rates between up- and down-flow pipes at different flow rates in the Double-U model

velocities. This is also an important insight for the state-of-the-art TRT evaluation, because one assumes constant radial heat flow over the depth when applying the line source approximation. Due to these results, the constant heat flow approximation is only true if the flow rate is high enough. 


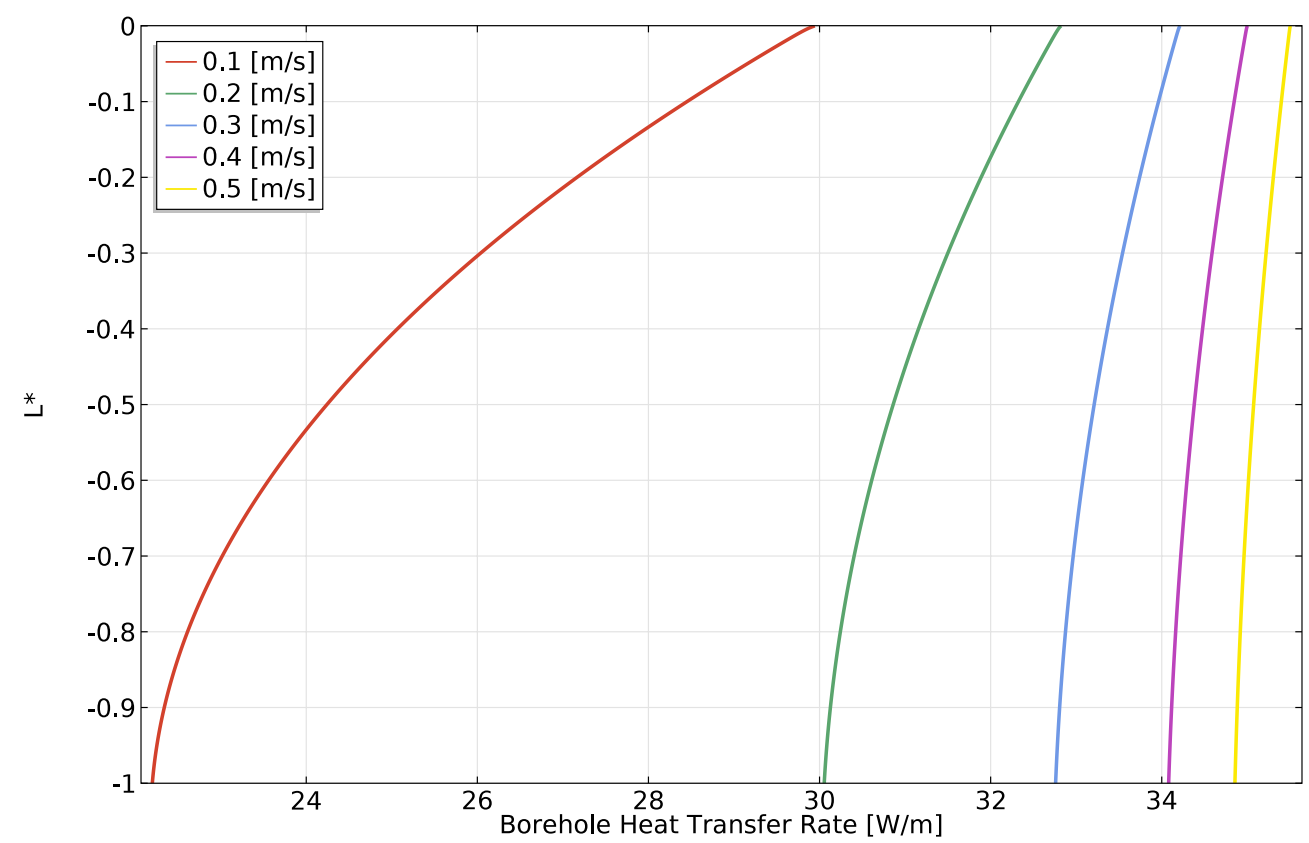

FiguRE 4.3: Heat transfers between the borehole and the subsurface heat at different flow rates in the Double-U model

\section{Short Timescale}

One main task of the presented work was to develop a numerical model that was accurate enough to reproduce the short-time behaviour of BHEs, which most simplified approaches do not include. The short-time reactions are also important for the new thermal response test add-on which is discussed below in Section 5 .

The model is set up equally to the configuration in the long-time scale consideration, but here the transient problem is solved. The depth-resolved results of the heat injection problem for two different pipe flow velocities, $0.1[\mathrm{~m} / \mathrm{s}]$ and $0.5[\mathrm{~m} / \mathrm{s}]$, are shown in Figure 4.4 and Figure 4.5. The presented graphs are the fluid temperatures for points in time that are multiples of the working fluid transit times, here labelled as $t_{0}$. In this way, one can follow the development process towards steady shape conditions of the pipe fluid. In both cases, slow and high velocity, the very early times are dominated by dynamic processes. The biggest amount of heat has gone into the ambient borehole, and at the bottom, the ambient temperature of $10\left[{ }^{\circ} \mathrm{C}\right]$ is almost reached. This is because the temperature difference between fluid and grout is maximal at the very beginning of the injection. After some more time, the temperature of the ambient increases, the heat transport slows down and the characteristic long-time profiles are already foreseeable after 10 fluid transits. Figure 4.6 points out the transient change of the integral heat 


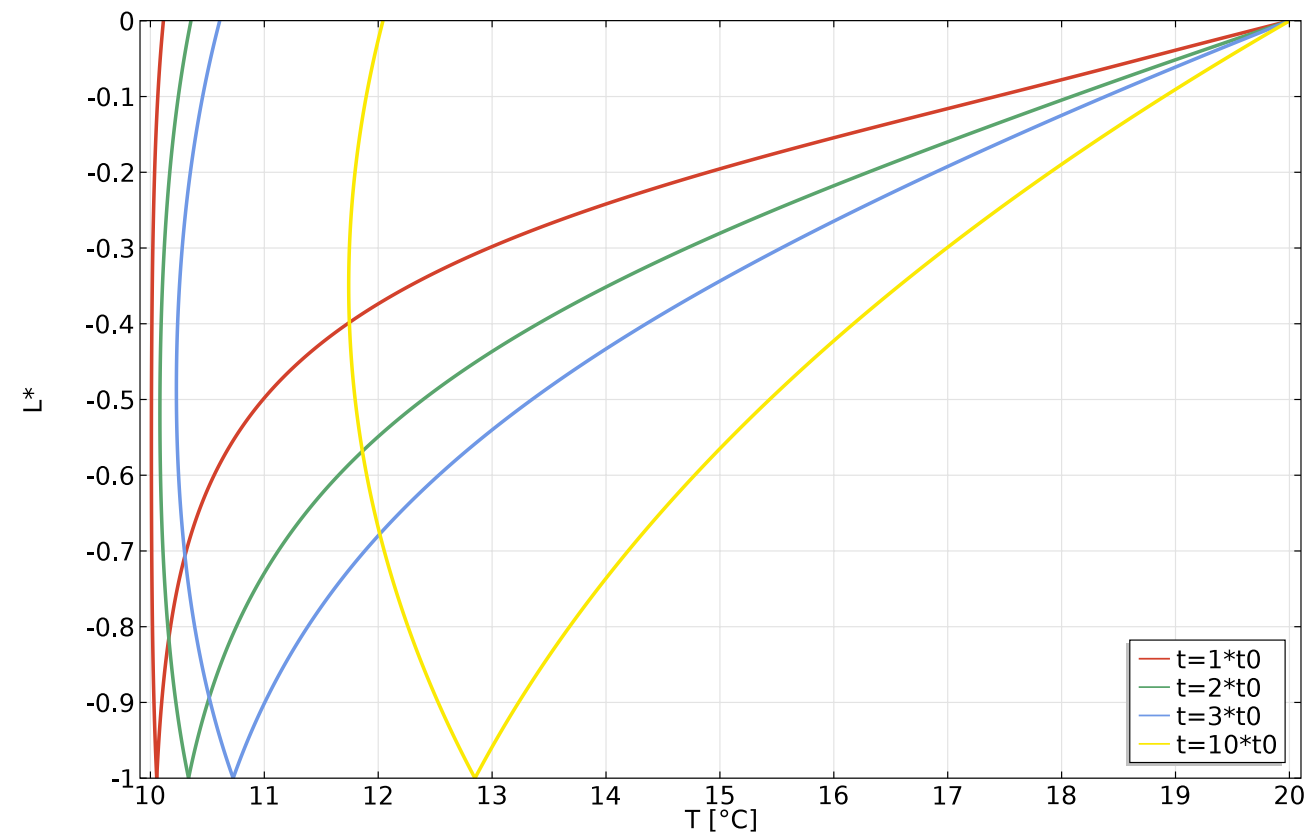

FiguRe 4.4: Fluid temperature for down- and up-flow branches at different times and $\mathrm{u}=0.1[\mathrm{~m} / \mathrm{s}]$ in the Double-U BHE model. The injection temperature is fixed to

$$
T_{\text {in }}=20\left[{ }^{\circ} \mathrm{C}\right]
$$

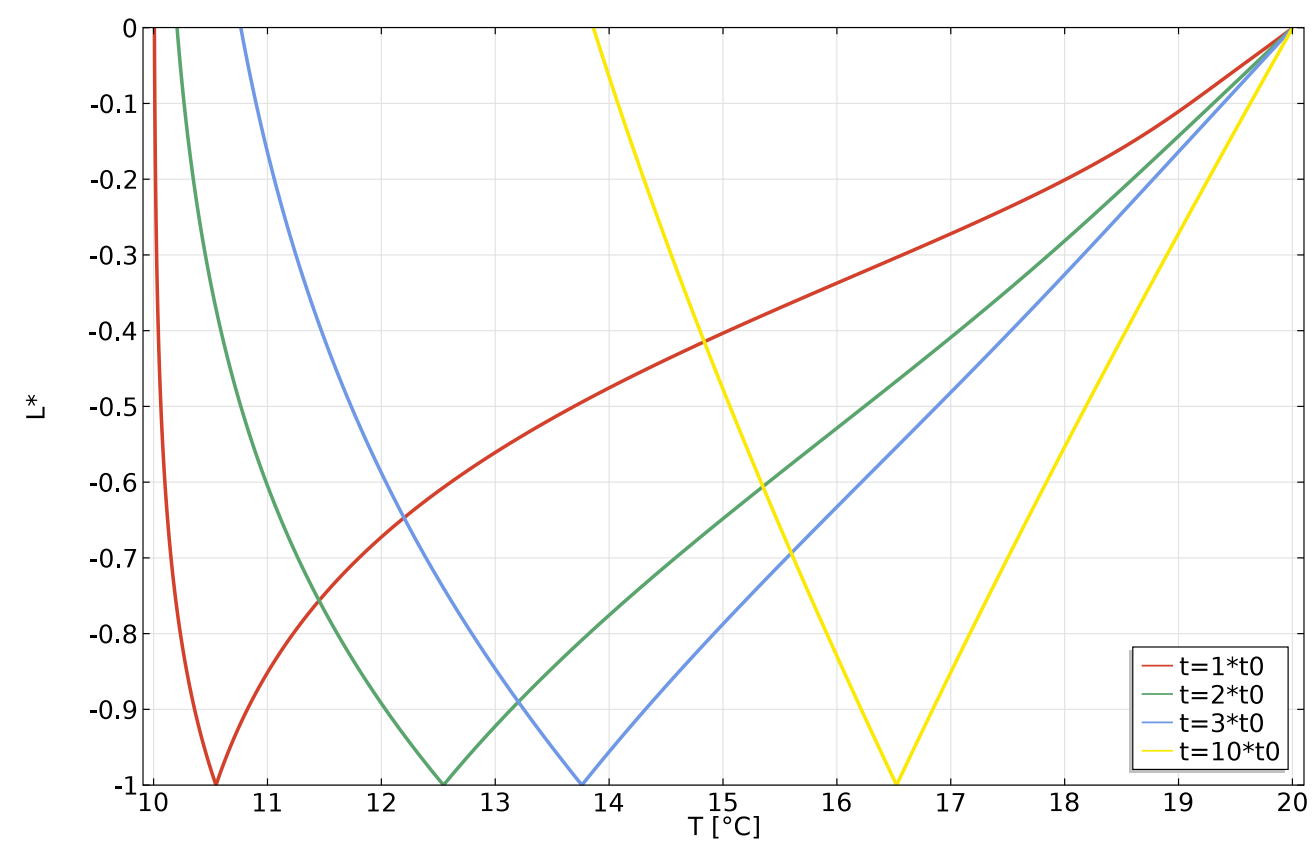

FigURE 4.5: Fluid temperature for down- and up-flow branches at different times and $\mathrm{u}=0.5[\mathrm{~m} / \mathrm{s}]$ in the Double- $\mathrm{U}$ BHE model. The injection temperature is fixed to $T_{\text {in }}=20\left[{ }^{\circ} \mathrm{C}\right]$ 


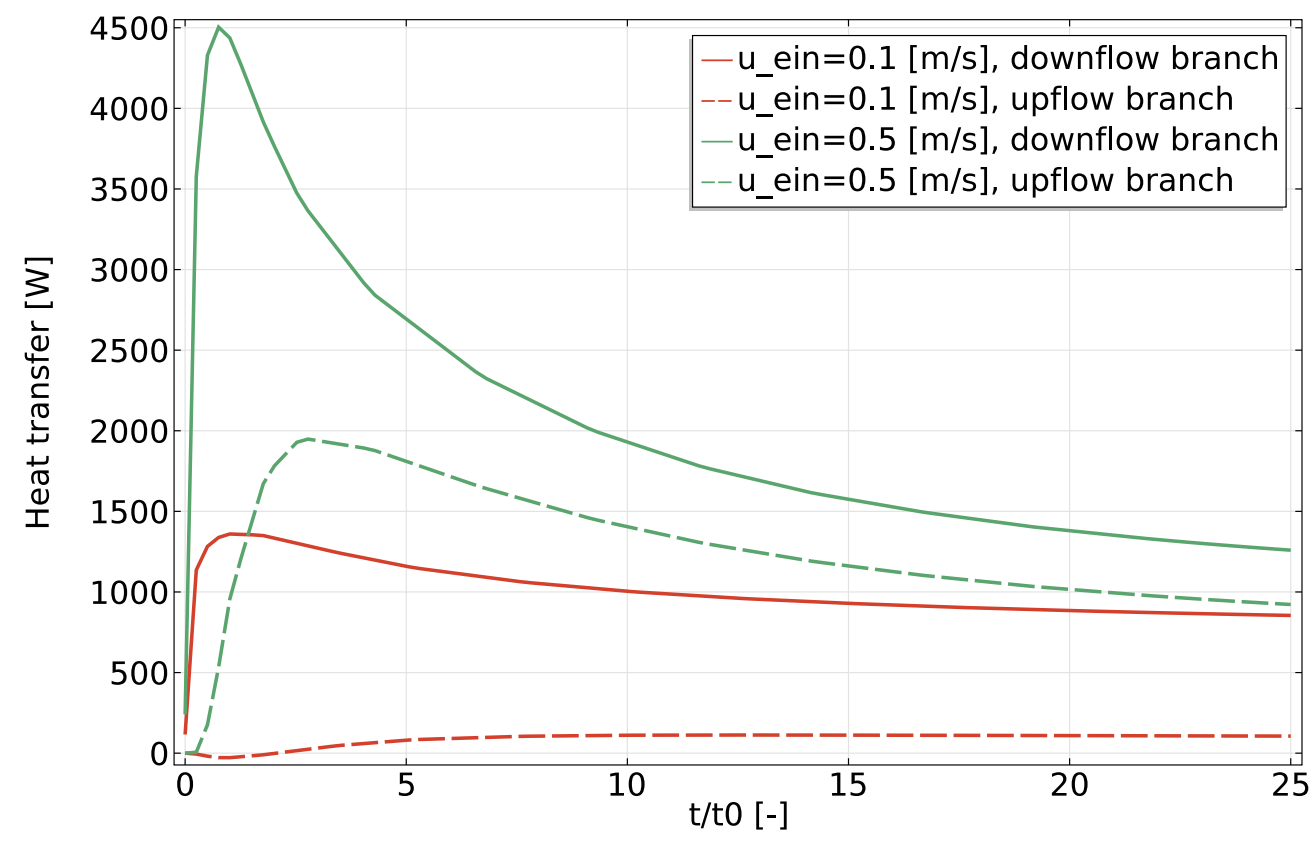

Figure 4.6: Development of integral normal boundary heat transfer of up- and downflow pipes at different flow rates in the Double-U model.

transfer rate of the up-flow and down-flow pipes. In both cases, the peak of maximum heat transfer is reached when the fluid reaches the bottom of the BHE. After that, the ambient area of the down-flow branches is warmer and the heat transfer decreases. The peak of the high velocity curve is much more distinctive because of the higher heat load that is injected during the short time interval. The heat transfer in the up-flow branch is from the beginning much smaller in the low-velocity case. Obviously, the temperature of the fluid reaches the ambient grout temperature level quickly, leading to a strong asymmetry between the up- and down-flow heat transfer. This underlines the deductions made above: low pipe fluid velocities lead to vertical heat flow distributions.

\section{Influence of Fluid Temperature Coupling}

As described in Section 3.2, the temperature dependencies of the properties of the working fluid are considered in the BHE models by bidirectional coupling between the parametric variables and the PDE systems. These dependencies are neglected in most of the simulation approaches that can be found in the literature. The reason is that the temperature differences, which appear during the TRTs and simulations, are usually assumed to be small, and thus the need for considering the impact of temperature caused 


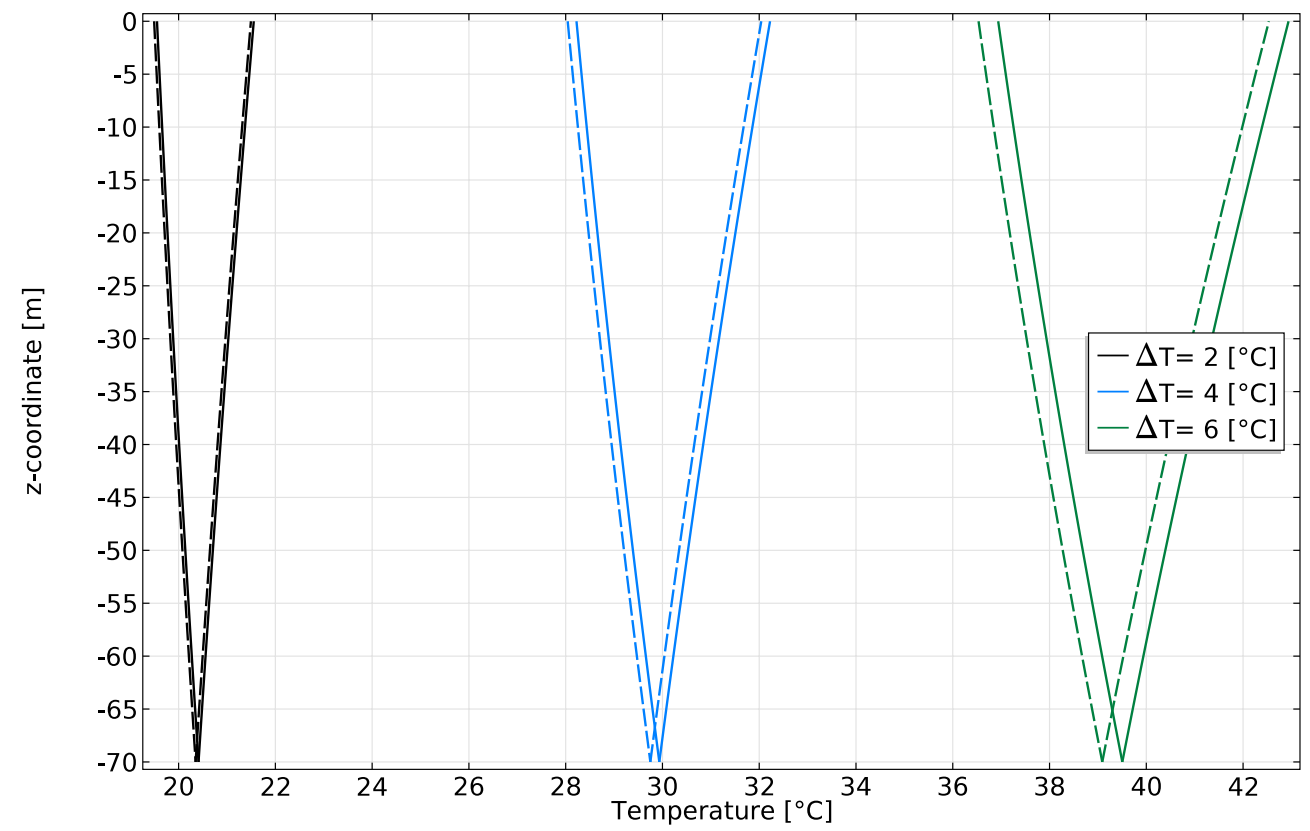

Figure 4.7: Comparison of fluid temperature profiles for constant (dashed lines) and temperature dependent (solid lines) fluid parameters and different inlet-outlet temperature pitches $\Delta T$, after $24[h]$ of heat injection

parametric changes is assumed to be negligible. In the following study, this impact on the results of a typical model run is investigated.

The numerical BHE system is stimulated by heat injection due to a fixed temperature difference between inlet and outlet. In one case, the inherent fluid parameters (density, heat capacity, thermal conductivity, viscosity) are set to the values according to a moderate temperature of $T=20\left[{ }^{\circ} \mathrm{C}\right]$ from the water-brine mix used at the ISFH test site and shown in B. In the other case, the temperature dependencies are taken into account, which is the usual practice in the modelling results that are presented in this thesis. Figure 4.7 shows the temperature profiles of the computational results after 24 $[h]$ of heat injection for the two different approaches and for different inlet-outlet temperature difference boundary conditions. It turns out that, as expected, the difference between the two approaches is low for small temperature differences and increased in case of a higher temperature spread. One reason for the higher difference is the fact that the overall temperature of the BHE is increased when a higher temperature spread, i.e. a higher injection rate, is applied.

The differences between the constant and temperature-dependent parameter approaches for different temperature spreads are quantified in Figure 4.8. The outlet temperature differences increase with time because the deviation from the selected temperature of 


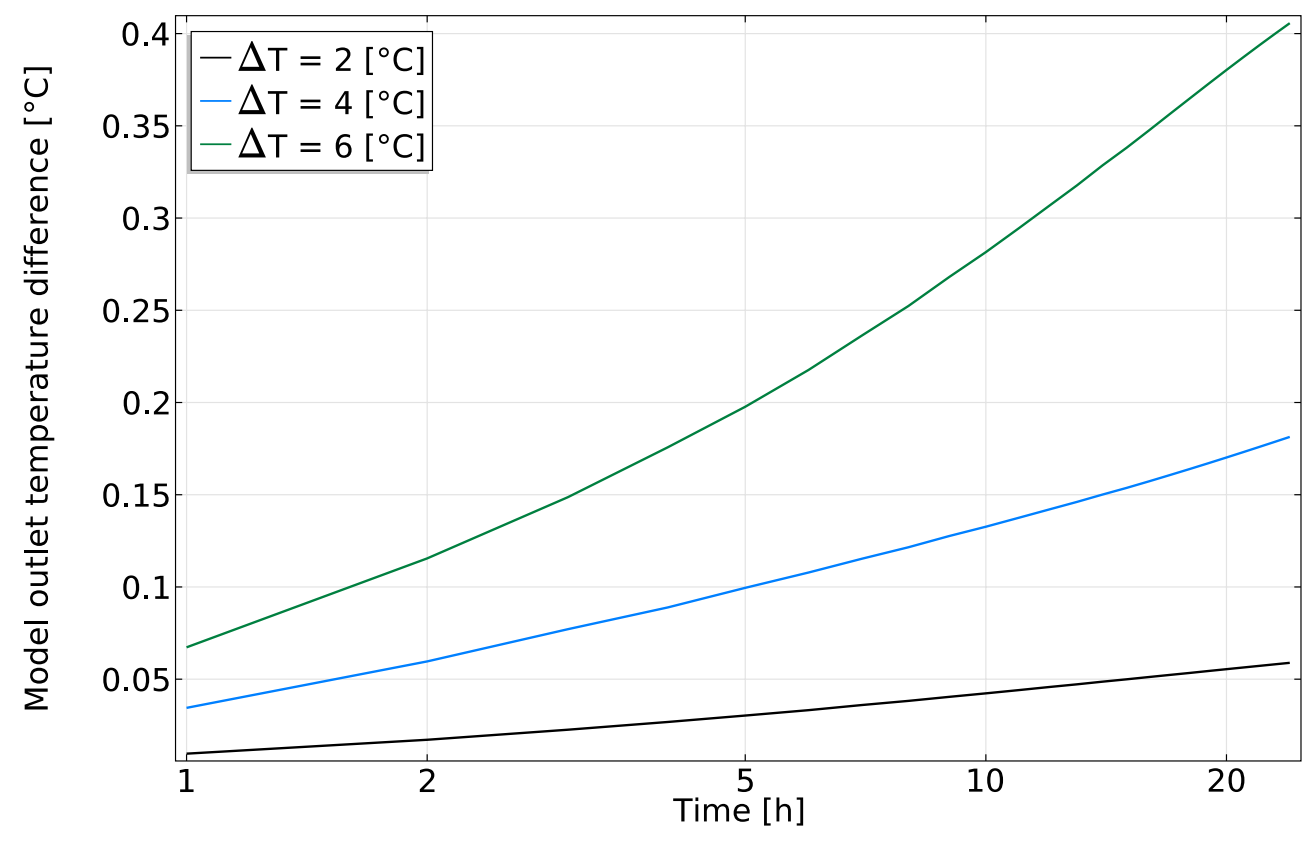

FiguRE 4.8: Differences of the outlet temperatures between constant and temperature dependent fluid parameter studies for different inlet-outlet temperature pitches $\Delta T$

the constant parameter approach $\left(20\left[{ }^{\circ} \mathrm{C}\right]\right)$ increases. The high heat injection rate that is induced by the high spread of $\Delta T=6\left[{ }^{\circ} \mathrm{C}\right]$ causes a temperature difference of about $0.4\left[{ }^{\circ} \mathrm{C}\right]$ after $24[h]$ of heat injection. Even in the case of the moderate 4 and $2\left[{ }^{\circ} \mathrm{C}\right]$ spreads, there is a measurable difference between the two approaches.

\section{Alternative BHE Designs}

The described principle of this full 3D Double- $U$ heat exchanger model is easily transferable to other designs that are used in shallow geothermal applications. Figure 4.9 shows a comparison of four different types of BHEs from Oberdorfer et al. [2011]. Additionally to the common Double-U system, the coaxial design is examined, a cross section is shown in Figure 1.1. The down-flow pipe is encased by a larger up-flow pipe. Recently, variations of the coaxial design have come on the market. Instead of one casing back-flow, there are several smaller pipes equally distributed around the centre downflow pipe. Two commercially available versions were implemented here: the Terrasonde (Terra Umweltwärmesonde GmbH [2011]) with six, and a similar design with twelve (Acuña and Palm [2011]) of the smaller up-flow legs. For the comparison study, the temperature at the inflow boundary is fixed to $T=0\left[{ }^{\circ} \mathrm{C}\right]$. The flow rate is kept constant for all designs, leading to different velocities and heat coefficients in the pipes due 


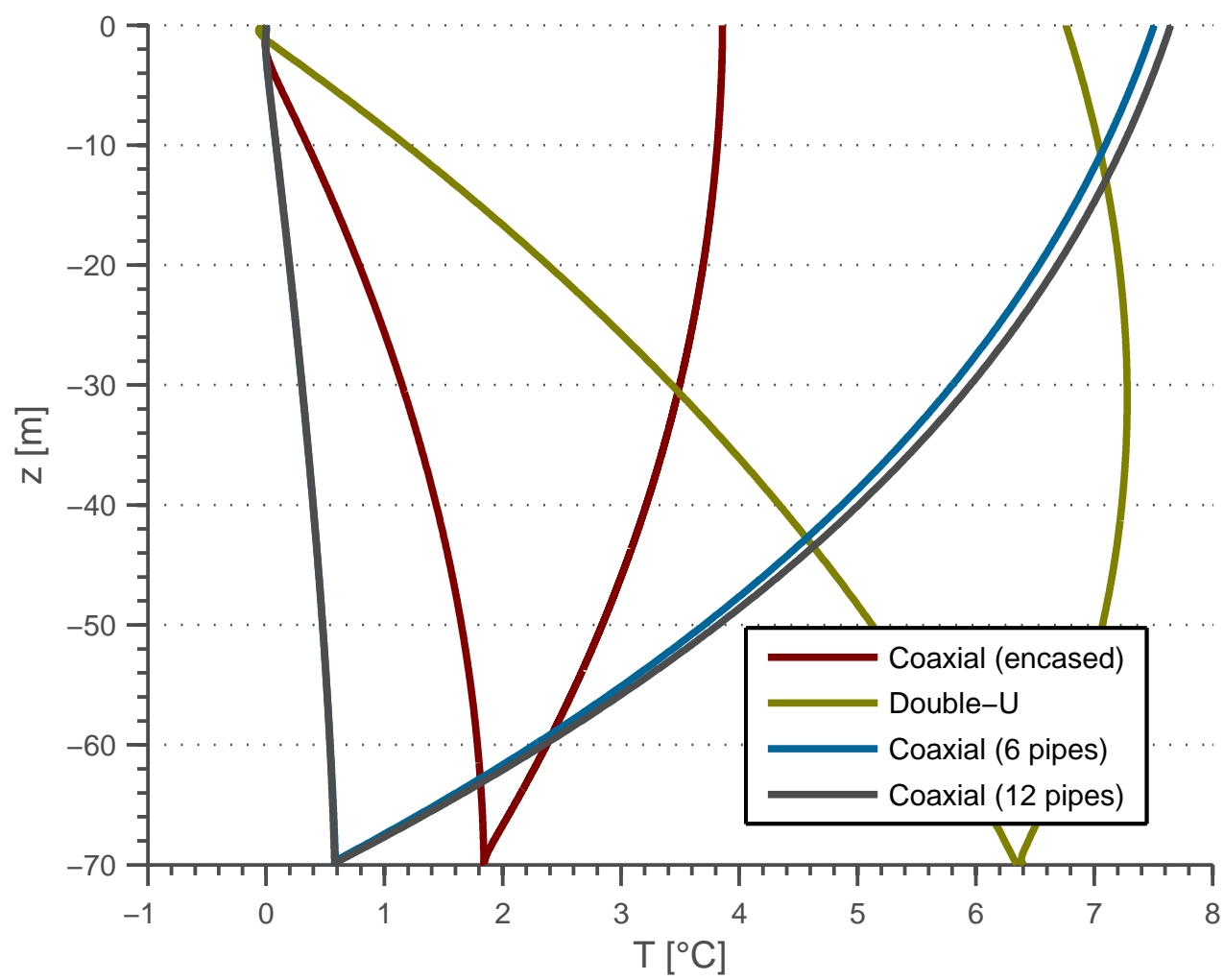

Figure 4.9: Comparison of the fluid temperature profiles of different BHE types after $3[h]$ of fluid injection with a temperature of $T_{i n}=0\left[{ }^{\circ} \mathrm{C}\right]$, from Oberdorfer et al. [2011]

to the different radii. All other parameters are equal for the four presented cases. In the figure, the resulting temperature profiles of the compared BHE types after $3[h]$ of cold fluid injection are presented. The down-flow pipes of both, the six- and twelve-pipe variations, have an isolating casing with a thermal conductivity that is reduced by the factor of ten compared to the conventional HDPE pipe material. Therefore, the temperature only increases very slowly during down-flow, and starts increasing rapidly at the up-flow branch due to the resulting high radial temperature gradient at the BHE bottom. The temperature profiles of both of the novel approaches are similar, but the fluid in the twelve-pipe approach becomes slightly warmer at the top as a result of their larger surface area. The cased coaxial pipe is not isolated and its top temperature is the smallest of all designs. This is related to two characteristics of this configuration: It comes up with i) the lowest surface area of all investigated pipe systems and ii) a high thermal connection between its up- and down-flow branches, leading to an increased thermal short-circuit, compared to the other designs. 


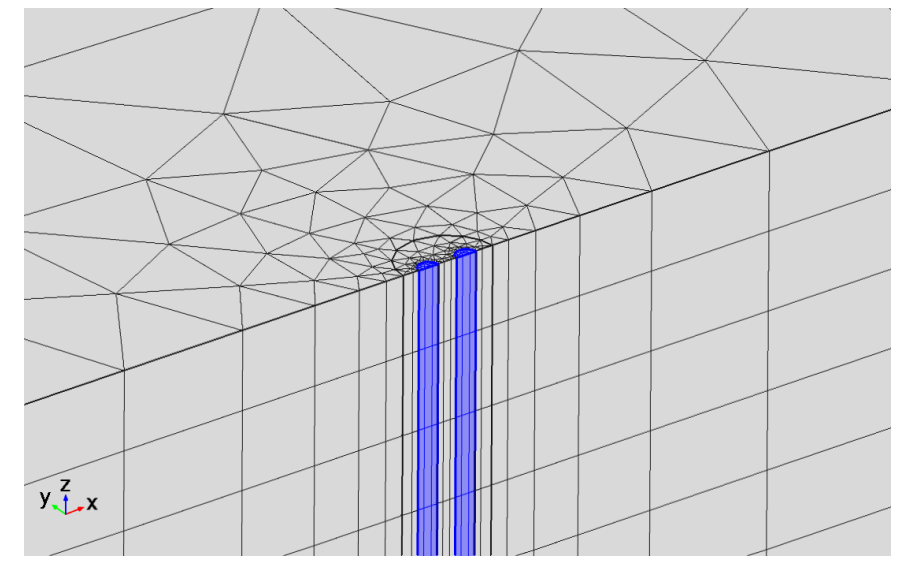

FigURE 4.10: Numerical model geometry, front plane with symmetry boundary conditions

\subsubsection{Comparison to an Experimental Sandbox Setup}

One general problem for any validation approach of BHE models is the lack of exact information about the thermal properties of any compared test site. The subsurface conditions are generally only roughly known. Core drilling samples, allowing for the laboratory investigations, give only local information that may already not be true a short distance away from the borehole. Even the grout filling quality within the borehole is a factor of uncertainty (see e.g. Riegger [2011]).

In 2000, a medium-scale laboratory setup of a grouted single U-tube was build at Oklahoma State University (Austin et al. [2000], Beier et al. [2003]). Because of its well known thermal properties and dimensions, it was recently used to make and publish very useful sets of reference data which are appropriate for the validation of BHE model settings (Beier et al. [2011]).

The setup consists of an $18[\mathrm{~m}]$ long wooden framed and sand-filled box with $1.8[\mathrm{~m}]$ side length and height. A highly conductive aluminium pipe is placed in the centre and acts as borehole. It is back-filled with grout and contains a standard single U-Pipe that is centred in the borehole by spacers. The box is furthermore equipped with thermistors that provide temperature measurements at specific locations in the surrounding sand. A heater can be used to heat up the carrier fluid by a constant rate. The reference data consists of two $50[\mathrm{~h}]$ thermal response tests, one with constant heat injection and one with an interruption sequence to study the short time reaction of the models. Figure 4.11 shows the heat supply for both tests. Because all necessary parameters and measurements are accessible, it is possible to adapt the numerical model to the laboratory setup 


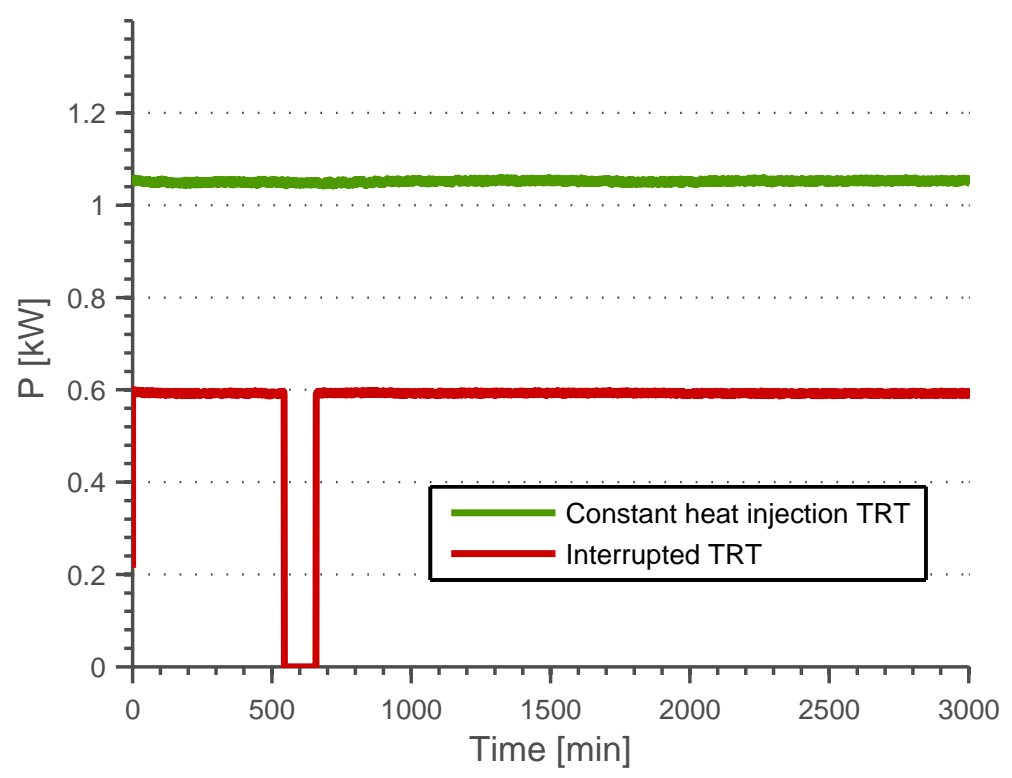

FIGURE 4.11: Heat injection powers for the constant and interrupted TRT

and to simulate the test runs. Due to the homogeneous conditions, the symmetry of the setup can be utilised by halving the geometry, as displayed in Figure 4.10. The resulting cut plane obtains symmetry boundary conditions $-\mathbf{n} \cdot(-\lambda \nabla T)=0$ or Neumann boundary conditions respectively. The time-dependent variables (temperature $T_{i n}$ at the entry of the pipe and flow rate $\dot{V}$ ) are taken as input, the mean fluid temperature $T_{\text {mean }}$ is compared. The flow rate is about $0.2[l / s]$, the resulting Reynolds number is between $1.2 e 4<R e<1.4 e 4$, depending on the temperature. The behaviour of the pipe flow in this range is turbulent, thus the calculated quantity of $N u$ is in the range of $N u \approx 100$. Figure 4.12 and Figure 4.13 show the results of the simulations in comparison to the sandbox data. Apparently, the deviations are too small to distinguish between the two lines. Besides a visible deviation right before the interruption phase in Figure 4.13, the experimental and numerical fluid temperatures match. Figure 4.14 and Figure 4.15 point out the deviations of the experimental and numerical results. The differences vary in the range of about $0.1[K]$ in both cases. The numerical model overestimates the mean fluid temperature in the first case and underestimates it in the interruption case. The uncertainties of some essential material parameters and measurements are additionally published $\left(\lambda_{\text {soil }}: \pm 5 \%, \lambda_{\text {grout }}: \pm 5 \%, \dot{V}: \pm 5 \%, P: \pm 1 \%\right)$. The red areas in Figure 4.14 and Figure 4.15 mark the ranges of the numerical results due to all of the mentioned uncertainties. The resulting range of deviation due to the uncertainties is small. Even the worst case estimation leads to temperature maximum deviations of $0.15[K]$ for the 


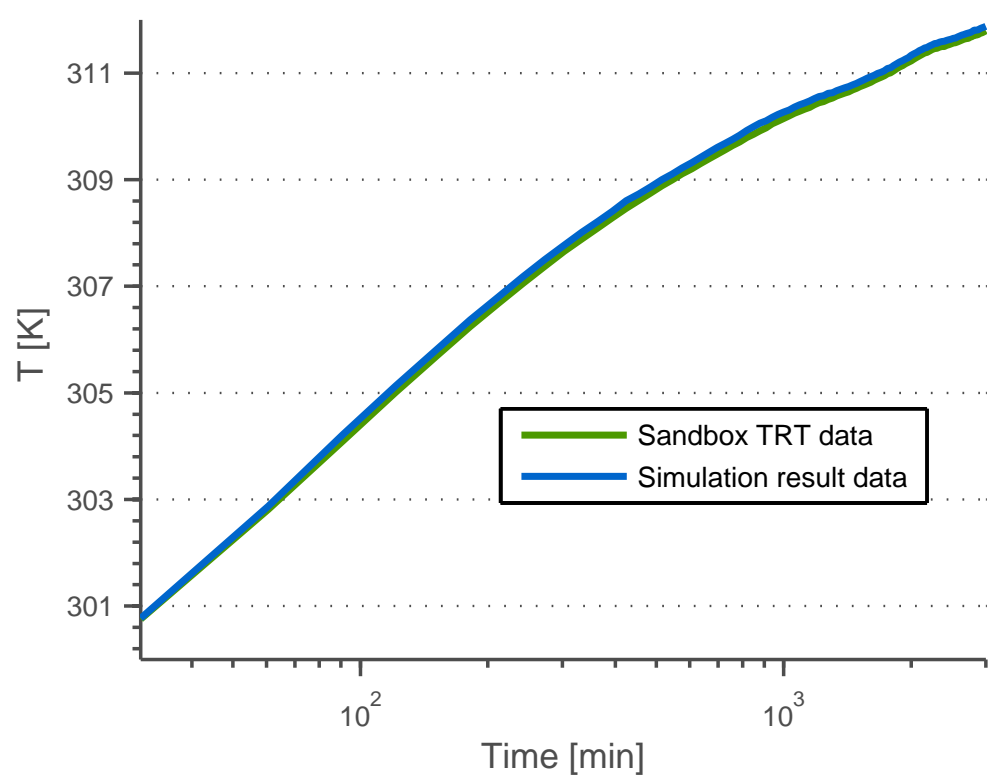

FIGURE 4.12: Mean fluid temperatures of the sandbox setup and numerical model for the constant heat injection run

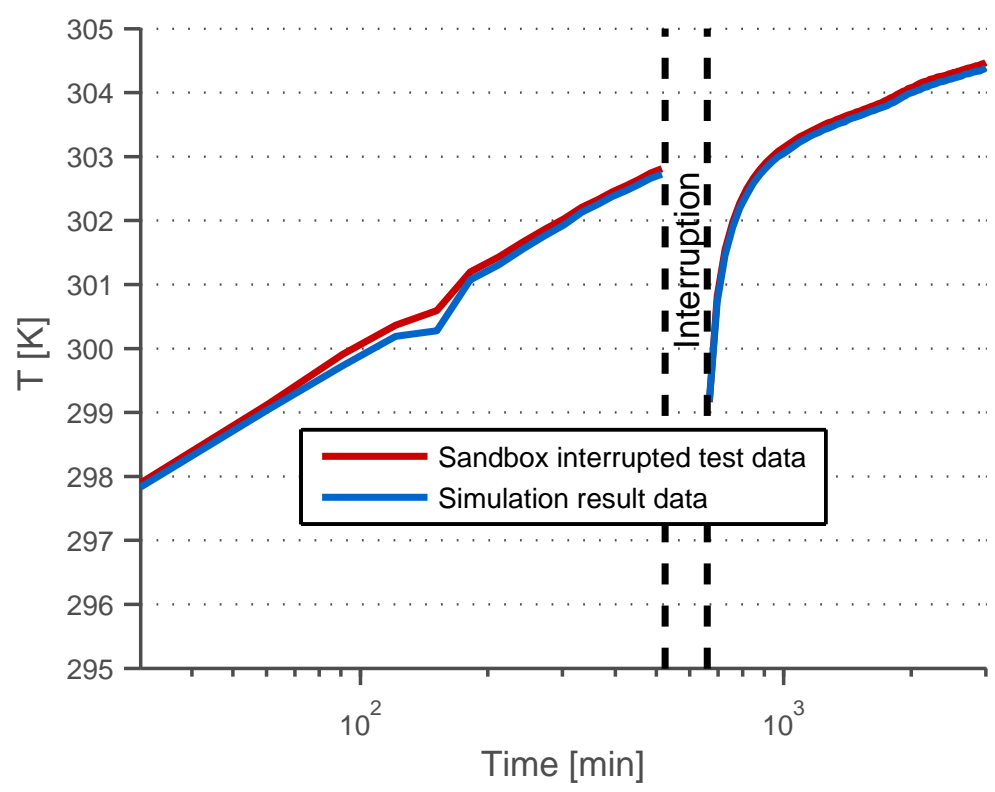

FIGURE 4.13: Mean fluid temperatures of the sandbox setup and numerical model for the interrupted heat injection run 


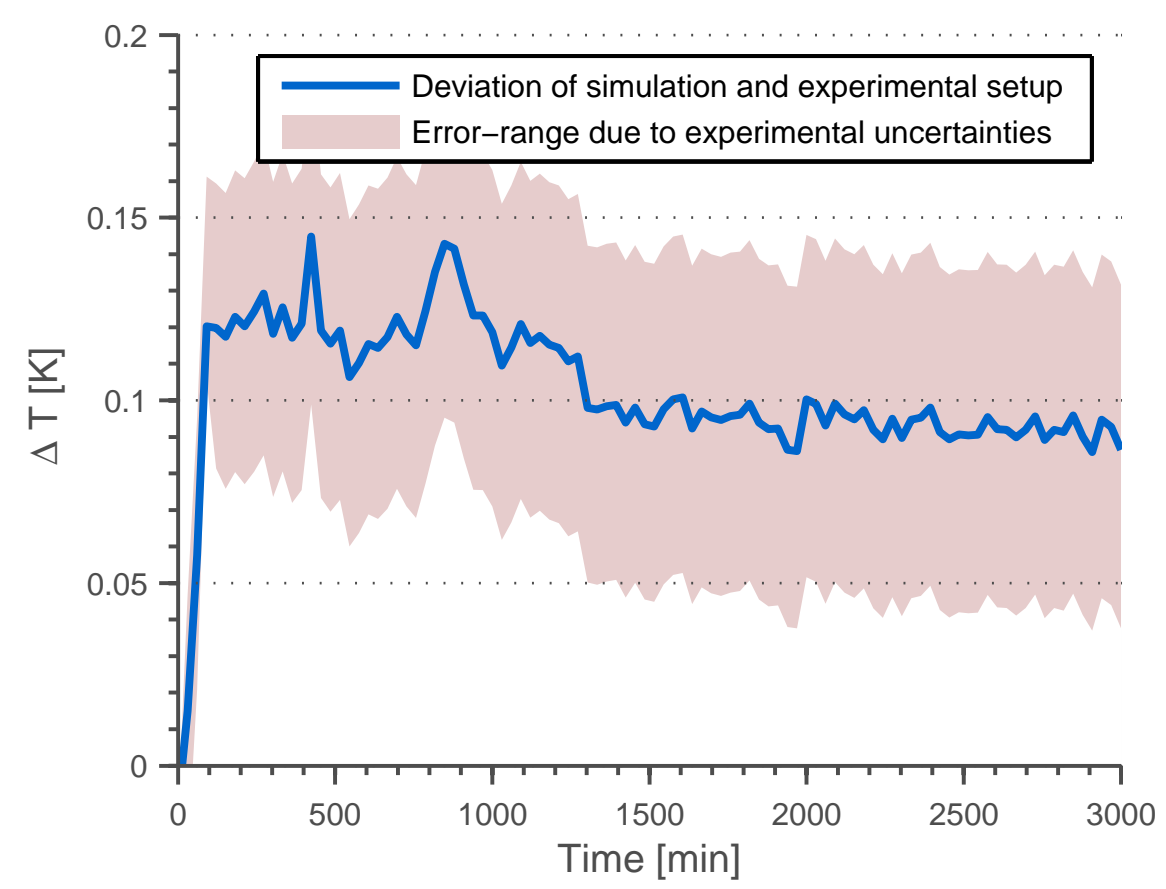

Figure 4.14: Deviation $T_{\text {num }}-T_{\text {exp }}$ between numerical model results and experimental data of the constant heat TRT

first experiment and $0.1[K]$ for the second experiment, respectively.

\subsubsection{Discussion}

The numerical BHE concept that was developed for this study has been validated extensively. The first step was focused on the general functionality of the model concept. Characteristic values, like the depth-resolved temperature profiles under different circumstances, were evaluated. The results are comparable to similar state-of-the-art modelling concepts from the literature. Just from the type curve results, it can be concluded that this model allows the accurate simulation of short and long timescale temperatures inside a Double-U heat exchanger and other design approaches.

The influence of the thermal dependence of the fluid thermal properties is an aspect of the BHE simulation that is often neglected. It could be shown that there is, however, an impact which becomes crucial when the temperatures deviate from the selected reference temperature for the fluid parameters, e.g. $20\left[{ }^{\circ} \mathrm{C}\right]$. Especially, a remarkable effect on long time predictions is anticipated according to the results, but also short-time computations may be influenced if the heat injection / extraction rates, i.e. the temperatures differences between the in- and out-flow branches, are high. 


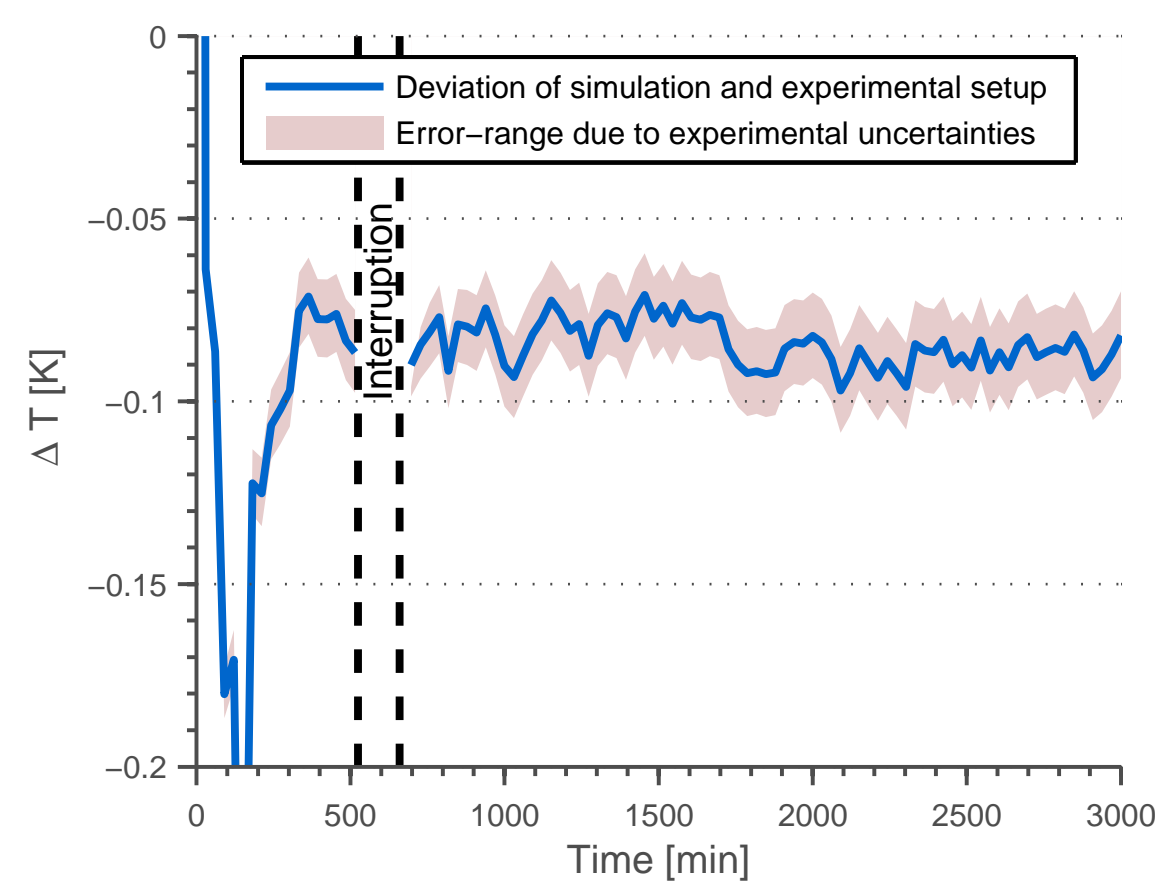

Figure 4.15: Deviation $T_{\text {num }}-T_{\text {exp }}$ between numerical model results and experimental data of the interrupted TRT

The comparison of the Single-U model to the experimental sandbox setup is convincing, the deviations between the numerical approach and the experimental results are very small for both long-time performance and short-time pulses.

Altogether, it can be concluded that the presented numerical model

- reproduces the elementary thermal short-time and long-time processes that appear in different kinds of BHE arrangements (Single-U, Double-U, cased and multi-pipe coaxial design),

- accurately reproduces and forecasts the thermal responses of experimental arrangements,

- is expendable up to any level of detail regarding geological inhomogeneities, subsurface flow coupling or further physical effects and

- is suitable as a benchmark model for simplified approaches and as a developing tool for further investigations. 


\subsection{Numerical Studies}

The results of the validation processes prove that the numerical concept provides reasonable results for the thermal processes in a shallow BHE and the ambient subsurface. One major advantage of numerical simulation is that, in principle, all the possible variables and processes of a simulation result are accessible. In addition, one can easily change the inherent parameters of the involved materials or geometric properties and scales. This is in contrast to the very limited access to the state conditions and measured values of experimental setups. Especially in the field of geothermal research, the interesting part of the experiment is located in the subsurface and thus very hard to access for measuring purposes. Even at very well-equipped test sites, such as the Geo-Solar-WP project site, the information density is still not as high as under laboratory conditions and not even roughly comparable to the possibilities of fully discretised numerical simulations. This advantage of the numerical approach is exploited to study thermal processes that appear in BHEs and their ambient subsurface under different conditions and the results are presented below.

\subsubsection{Parametric Variations}

In this section, the results of various parametric studies that were performed with the numerical model are presented. The following parametric study results are partially published in Oberdorfer et al. [2013b]. Here, the Double-U model is used because of its worldwide relevance in applications and especially in Europe. The main question of the studies is about how the efficiency of a BHE can be improved. In general, the criterion for the efficiency is the borehole's thermal resistance $R_{b}$. This value accumulates all thermal resistances between the working fluid and the subsurface and, thus, a decreased $R_{b}$ results in an improved thermal contact.

Generally, the experimental in-situ determination of $R_{b}$ is done after evaluating the effective thermal conductivity $\lambda_{\text {eff }}$ from Equation 2.23 and replacing $T_{b}$ using Equation 2.24. Due to this derivation, $R_{b}$ is only accurate within the line source approximation limitations, as discussed in Section 2.3. One advantage of the numerical model is that there is full access to all state variables and, thus, $R_{b}$ can be determined exactly due to its definition as the temperature difference between working fluid and subsurface, divided 
by the heat flow rate between the surfaces of the pipes and the BHE. The temperatures are calculated numerically by integration over the whole borehole surface and integration over the pipes volumes, respectively. The numerical approach offers the possibility of performing changes of any desired parameter and of studying the effect on the BHE performance in terms of $R_{b}$.

A benchmark model run was performed using the parameters shown in Table 4.1, that correspond to the values from the experimental test site described in Section 3.1. The model run conditions are analogous to a thermal response test: Heat is injected at a constant rate of $4.9[\mathrm{~kW}]$ for $24[h]$. The heat injection rate is controlled by measuring the mean BHE fluid outflow temperature and adapting the corresponding inflow temperature in every numerical time step.

The results are presented in terms of the effects of parameter changes on the borehole's thermal resistance. In every figure, the change of $R_{b}$ due to a parameter variation is shown. $R_{b}$ and the corresponding parameter are divided by the benchmark model values to make the different studies comparable (relative quantities are marked with asterisks: $\left.R_{b}^{*}=R_{b} / R_{b, \text { benchmark }}\right)$. The evaluated value of $R_{b}$ from the benchmark run is $R_{b}=0.09\left[m K W^{-1}\right]$. This is in the range of the TRT evaluation results from the test site, where the values for the three BHEs vary between 0.071-0.117 $\left[\mathrm{mKW}^{-1}\right]$ (see also Table 3.2).

\begin{tabular}{lrllll}
\hline & Parameter & Reference value & Min & Max & Unit \\
\hline Borehole depth & $L$ & 70 & 7 & 140 & {$[\mathrm{~m}]$} \\
Pipe radius & $r_{p}$ & 13.1 & 7.86 & 26.2 & {$[\mathrm{~mm}]$} \\
Pipe distance & $d$ & 61 & 30.5 & 103.7 & {$[\mathrm{~mm}]$} \\
Borehole radius & $r_{b}$ & 95 & 76 & 228 & {$[\mathrm{~mm}]$} \\
Fluid therm. cond. & $\lambda_{f}$ & 1.71 & 0.1 & 10 & {$\left[\mathrm{Wm} \mathrm{m}^{-1} \mathrm{~K}^{-1}\right]$} \\
Pipe therm. cond. & $\lambda_{p}$ & 0.28 & 0.1 & 10 & {$\left[\mathrm{Wm}^{-1} \mathrm{~K}^{-1}\right]$} \\
Grout therm. cond. & $\lambda_{g}$ & 2.00 & 0.1 & 10 & {$\left[\mathrm{Wm}^{-1} \mathrm{~K}^{-1}\right]$} \\
\hline
\end{tabular}

TABLE 4.1: Parameters of the benchmark model run and variation ranges of the parametric studies

The thermal conductivities of the working fluid, the pipe material and the grout filling are varied and the impact on $R_{b}^{*}$ is shown in Figure 4.16. All of the resulting curves indicate a reciprocal relationship between the particular conductivity and the resistance which is consistent, because every individual element that is toggled between the working 


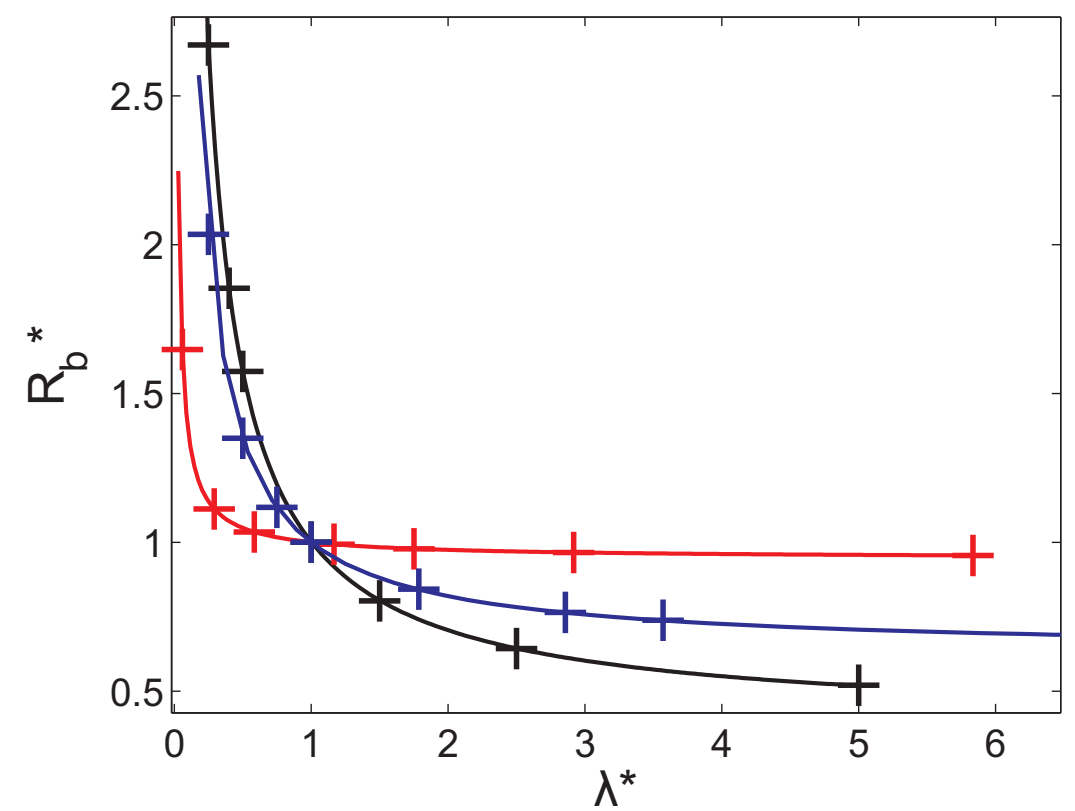

FiguRE 4.16: Thermal resistance in dependence of the involved domain thermal conductivities; Working fluid (red), pipe material (blue) and grout filling (black)

fluid and the subsurface can be seen as part of a serial resistance network ${ }^{2}$, and of course, the thermal resistance goes towards infinity if any one of the involved conductors of the network series goes towards zero.

The highest impact is observed at the thermal conductivity of the grout material. The joint resistance is significantly reduced when this parameter is increased above the reference value and causes a heavy increase of $R_{b}^{*}$ when it is decreased below the reference. This strong effect of $\lambda_{g}^{*}$ is assumed to be due to its high amount of thermal mass compared to other elements in a BHE. The thermal conductivity of the working fluid $\lambda_{f}^{*}$ has only a very low impact on $R_{b}^{*}$. The reason for this is the fact that the major part of heat transport in the pipes is advective because of the turbulences of the pipe flow and, thus, the diffusive part is rather negligible. As is to be expected, the influence of the pipe material thermal conductivity $\lambda_{\text {grout }}^{*}$ lies between the other two.

The parametric study results are fitted to analytical functions to survey the inverse relationship between the thermal conductivities and $R_{b}^{*}$. The results are outlined in Table 4.2. The fit results underline the plausibility of the assumption of a reciprocal linear relationship between the single thermal conductors and the joint resistance of the BHE. This can be concluded because the exponents $\beta_{3}$ from the polynomial fits are close

\footnotetext{
${ }^{2}$ As it is described and used e.g. by Bauer [2011]
} 


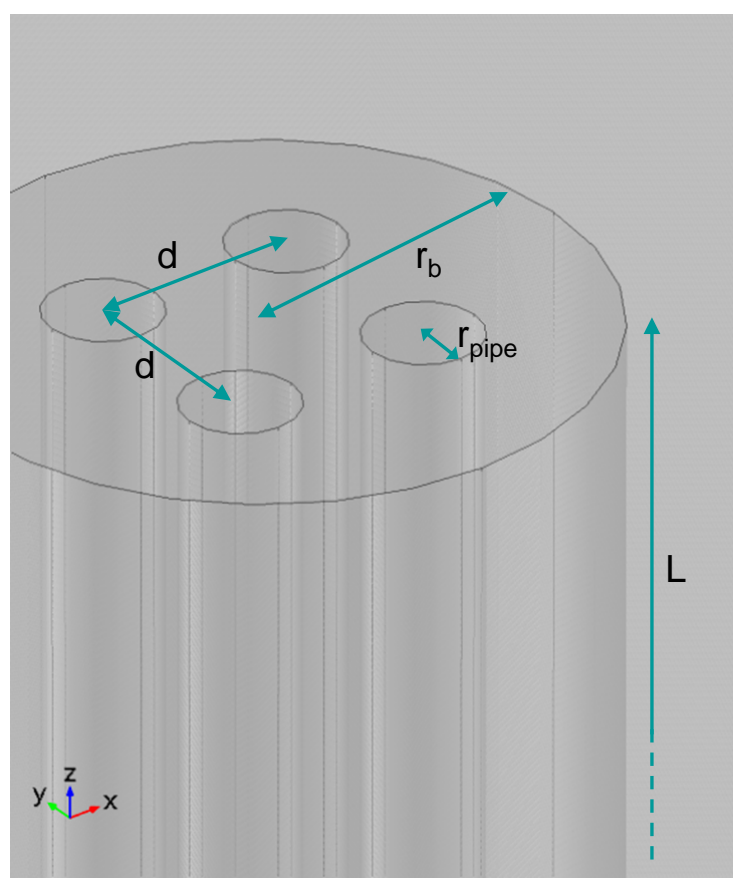

Figure 4.17: Geometric quantities of the BHE that are varied in the study

to 1 and the Mean Squared Error (MSE) value is low. However, $\beta_{3}$ is slightly smaller in all three cases and, thus, there must be processes that overlap the pure reciprocal relationship. An explanation can be found in the fact that the down-flow and up-flow pipes thermally interact with each other and cause thermal short cuts.

Figure 4.18 shows results from parametric studies where the dependence of $R_{b}^{*}$ on three selected geometric quantities is determined; the BHE length $L$, the borehole radius $r_{b}$ and the distance between the pipes $d$ are varied. The black data points represent the dependence of the thermal resistance on the length of the BHE. The values of $R_{b}^{*}$ do not change when $L$ is varied. The reason for this is that the heat injection rate per unit length $q$ is kept constant in all cases. This is an interesting result because even though the radial heat flux through the BHE is not constant in axial direction due to different borehole temperatures in different depths, as shown in Section 4.1.1, the mean value of

\begin{tabular}{lrrrr}
\hline Thermal conductivity & $\beta_{1}$ & $\beta_{2}$ & $\beta_{3}$ & MSE \\
\hline$\lambda_{f}^{*}$ & 0.94 & 0.06 & 0.89 & $1.9 \mathrm{e}-10$ \\
$\lambda_{p}^{*}$ & 0.63 & 0.37 & 0.96 & $1.1 \mathrm{e}-06$ \\
$\lambda_{g}^{*}$ & 0.38 & 0.62 & 0.94 & $3.3 \mathrm{e}-06$ \\
\hline
\end{tabular}

TABLE 4.2: Fit results of the parametric studies in Figure 4.16 to the reciprocal linear function $R_{b}^{*}=\beta_{1}+\beta_{2} \lambda^{-\beta_{3}}$. 
the thermal borehole resistance per unit length is independent of the borehole length. This means that the axial heat flux profile is stretched when the borehole is deeper, but it does not change its shape.

The influence of the borehole radius on the borehole's thermal resistance is represented by the blue data points. $r_{b}$ has a lower limit, the borehole radius must at least be large enough so that the pipes are still entirely inside the BHE. Theoretically, there is no upper limit for the borehole radius, but of course there are practical restrictions. $R_{b}$ increases with increasing $r_{b}$ because of the additional thermal-resistive grout mass between the pipes and the subsurface, but the curve slope decreases for larger borehole radii.

The distance between the BHE pipes $d$ is varied within the possible range inside the borehole. $d$ must at least be large enough to avoid touching of the single pipes and, furthermore, the proper construction of a numerical mesh between the pipes must be granted. The upper limit is the value for $d$ right before the pipes reach the borehole boundary. The red curve in Figure 4.18 shows the effect of the distance of the pipes inside the borehole. An increased distance decreases $R_{b}$ considerably, it is only half as large when the two extreme cases, pipes in contact with each other and pipes outside close to the borehole surface, are compared. This result matches to the conclusions from the work of Acuña and Palm [2009] who solved the BHE heat transfer problem numerically for two dimensional BHE cross sections and found that "the best U-pipe BHE configuration corresponds to when the pipes are completely apart from each other". Although Single-U pipes were examined for their study, the results are comparable due to the symmetry of these two problems.

In general, the heat pipes for closed loop geothermal applications are only available at a very limited number of different diameters which is due to the limited number of industrial production standards in this area. Therefore, there only exist experimental results and data for the few available sizes of pipes. The numerical investigation offers the possibility of having a free choice concerning the pipe radii. One only has to consider the following restrictions: The pipes must be thick enough to provide a reasonable meshing inside and, furthermore, it makes sense to make the pipes not too thin to keep the pressure drop between the in- and outlets at a reasonable value. Therefore, the pipe 


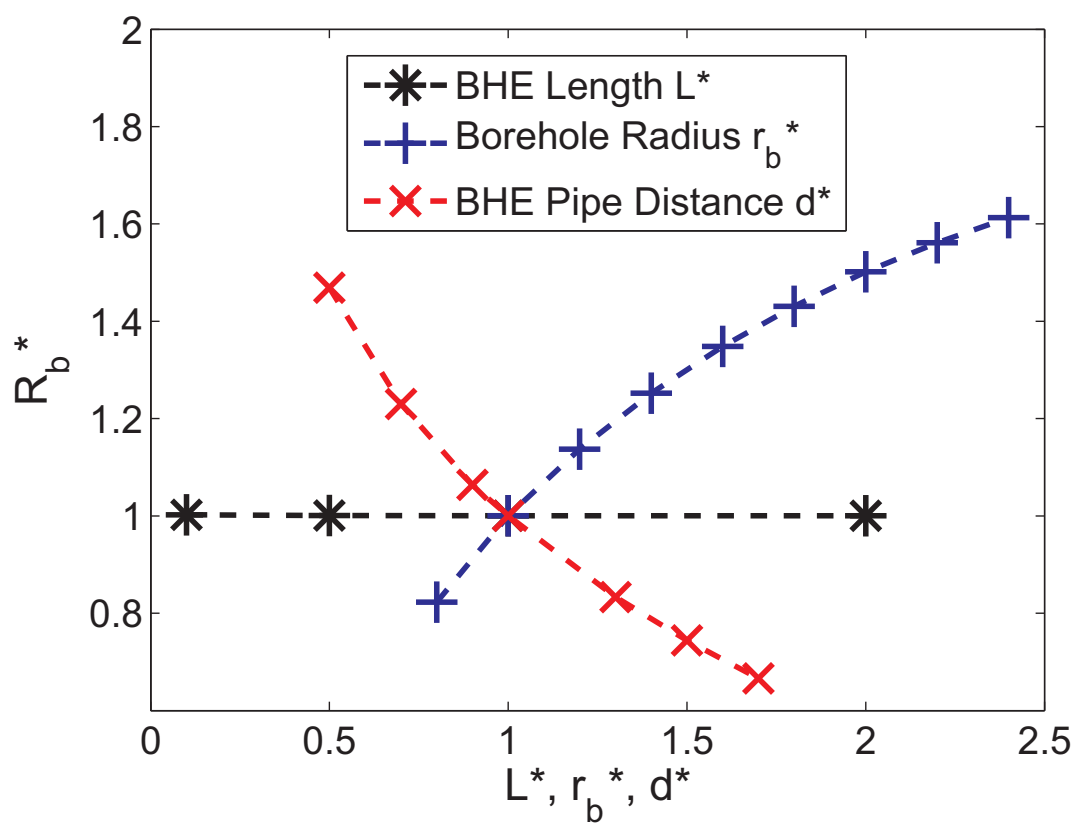

FiguRE 4.18: Thermal resistance in dependence of different geometric BHE proportions

radius is varied between $0.6-2 \cdot r_{p}$ for this study and the impact on $R *_{b}$ is shown in Figure 4.19.

The curve progression of the resulting reliance between $r_{p}^{*}$ and $R_{b}^{*}$ is very interesting: For small radii, the thermal resistance decreases with increasing radius due to (i) the increasing surface of the pipes which causes more heat exchange area and (ii) the smaller distance between the pipes to the borehole boundary. The latter is just a comparable effect to an increasing pipe distance, as discussed in the study above. However, if $r_{p}^{*}$ is further increased, $R_{b}^{*}$ also increases again slightly, resulting in a minimum of $R_{b}^{*}$ at $r_{p}^{*} \approx 1.7 \cdot r_{p} \approx 22.3[\mathrm{~mm}]$. A closer look at the heat flux circumstances helps to explain this local minimum of $R_{b}^{*}$. The integrated normal heat fluxes through the pipe walls and the borehole are plotted in Figure 4.20. As already mentioned, the radial pipe heat flow rises with increasing pipe radius. Yet, the radial heat flow through the borehole surface does not change significantly. The heat flow through the pipes becomes larger than the heat flow between BHE and subsurface, and this is only possible if heat flows directly between the up- and down-flow branches of the pipes. This arising thermal short cut is fatal for the system performance and leads after all to a rising borehole thermal resistance. 


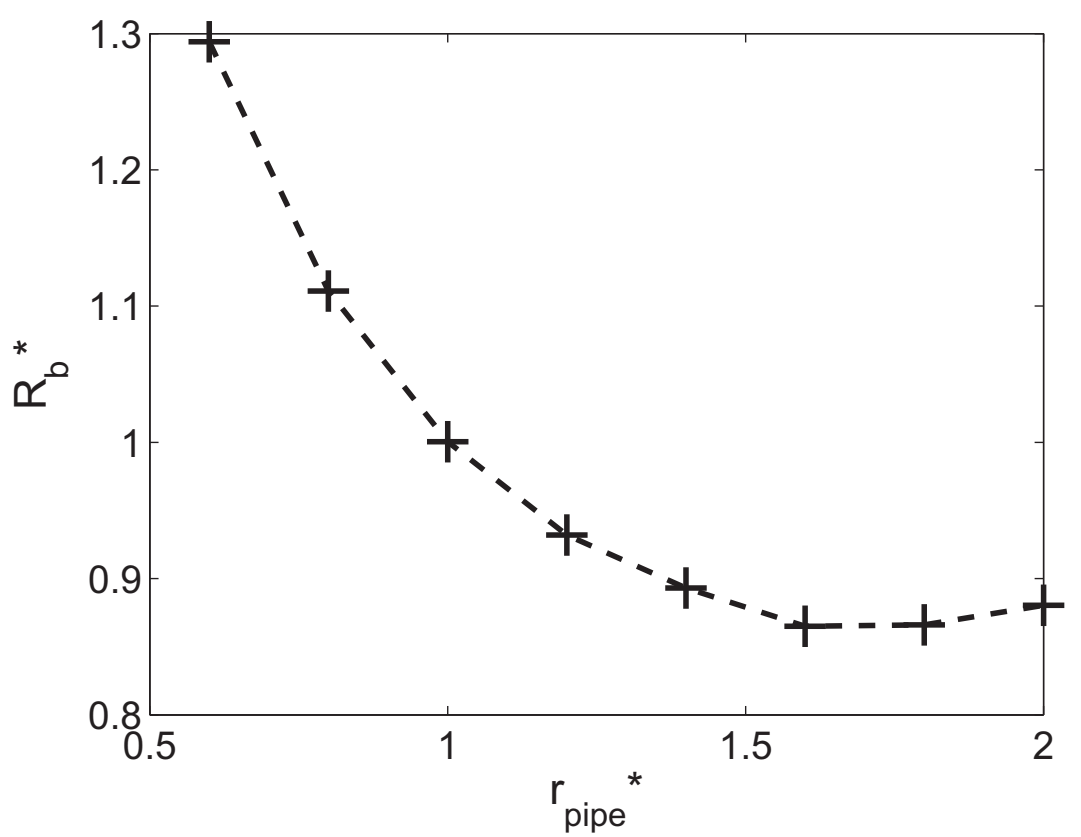

FIGURE 4.19: Thermal resistance in dependence of the pipe radius

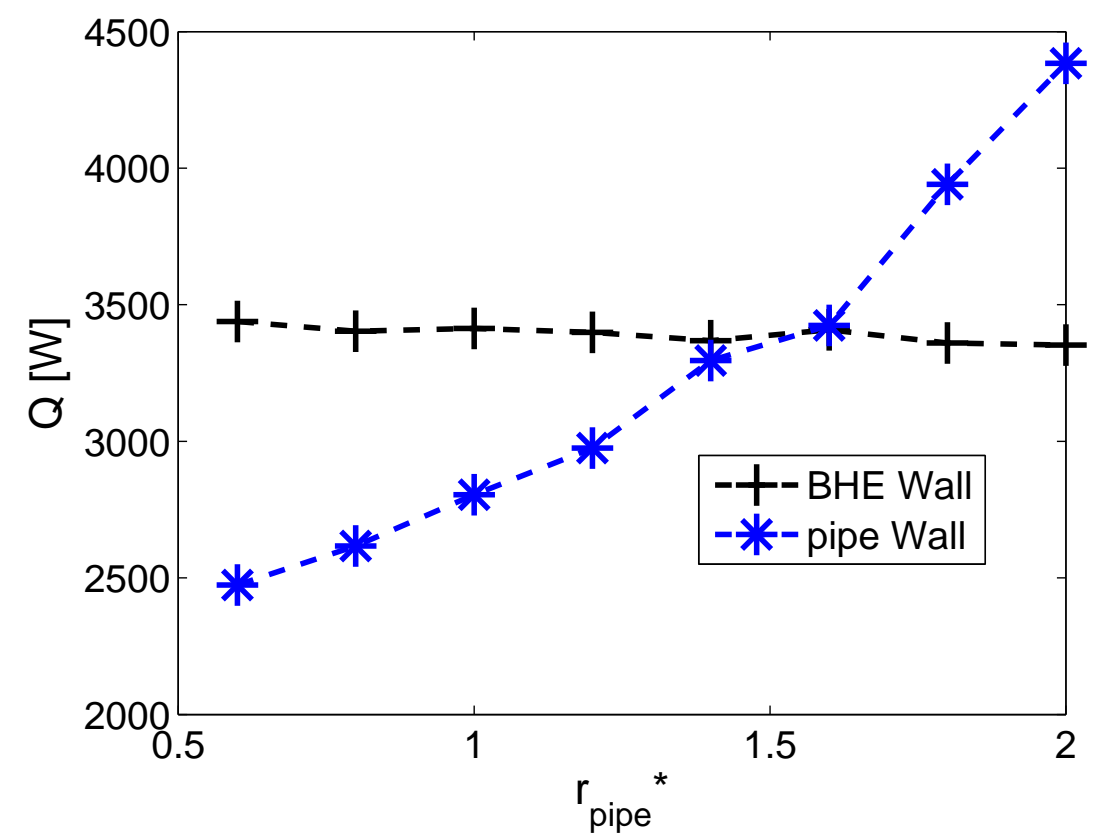

FiguRE 4.20: Integral heat flux trough the pipe walls and through the borehole wall in dependence of the pipe radius 


\subsubsection{Influence of Groundwater Flow}

In many cases, the drillings for shallow geothermal applications penetrate one or more aquifers. The influence of the effects of groundwater flow on the heat transfer characteristics of BHEs is a very important subject of interest, because (i) the increased heat transport due to groundwater flow may under some circumstances allow for the reduction of the required borehole depth and (ii) the mid- and long-time interaction between multiple BHEs, that are arranged in short distances from each other, can be significantly affected.

In this section, the results from a TRT study are presented, in which horizontal groundwater flow influences the heat transport in the porous medium around the BHE. The thermal and hydraulic parameters for the study are chosen due to the ISFH test site conditions. The velocity field in Equation 2.1 is coupled to the velocity field in the equation solving the subsurface flow in porous media PDE. An artificial horizontal hydraulic gradient is applied to the model region, causing a subsurface flow regime around the borehole. The velocity magnitude depends on the hydraulic gradient due to Darcy's Law, the hydraulic conductivity $K[\mathrm{~m} / \mathrm{s}]$ was gained in situ from pumping tests within the Geo-Solar-WP project. The groundwater table at the test site is around $28[\mathrm{~m}]$ below the soil surface. Figure 4.21 shows the temperature distribution in three different depths after $24[h]$ of heat injection at a constant rate of $2[k W]$. In the upper cross section, representing the unsaturated zone above the groundwater table, the temperature distribution is radial symmetric while in the deeper aquifer, heat is transported downstream.

The influence of the subsurface flow on the performance of the BHE is examined in a parametric study. Therefore, the Darcy velocity is varied by adjusting the hydraulic gradient in the subsurface model domain. For this parametric study, the groundwater level is set to $0[m]$. Thus, the BHE is completely surrounded by the aquifer. The effect on the mean pipe fluid temperature is outlined in Figure 4.22 which shows a TRT evaluation like diagram, the temperature is plotted against the logarithmic time scale. Up to about three hours of heat injection, there is no effect on the temperature development. Only after longer times can an effect be observed and it is more distinctive for higher groundwater velocities. 


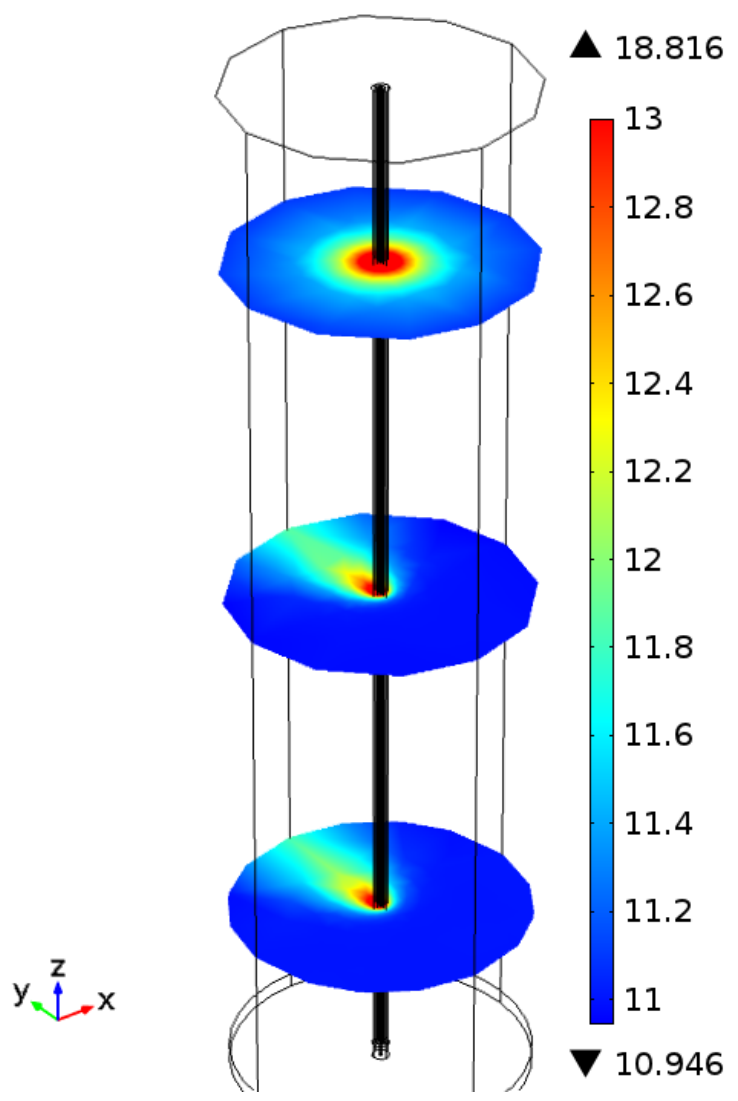

Figure 4.21: Temperature distribution of combined TRT - groundwater flow model. Darcy velocity: $u_{\text {Darcy }}=0.1\left[m d^{-1}\right]$, heat injection rate: $P=2[k W]$, groundwater table: $30[\mathrm{~m}]$

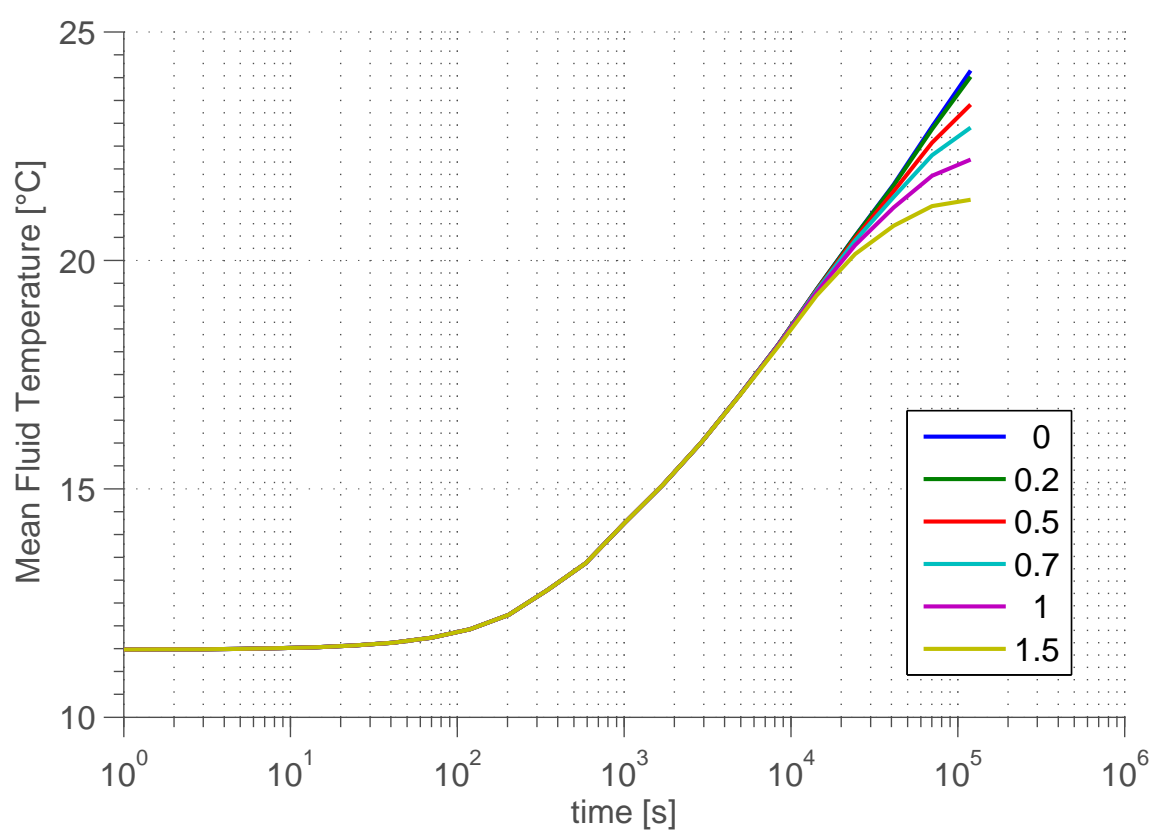

Figure 4.22: Time development of the fluid mean temperature for different groundwater flow velocities 


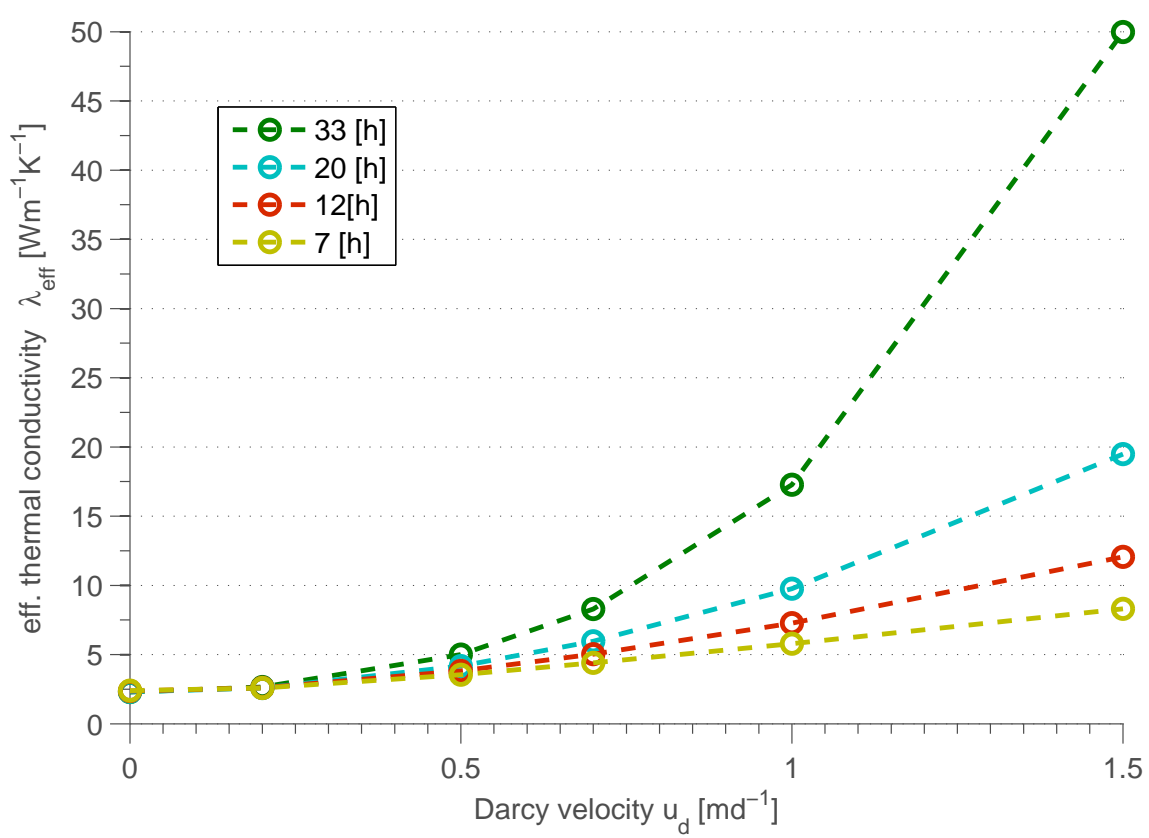

FIGURE 4.23: Effective thermal resistance (due to TRT evaluation) in dependence of groundwater flow velocity

In Figure 4.23, the impact on the temperature slope is quantified by evaluating the effective thermal conductivity which is, due to the classical TRT evaluation, proportional to the slope. It is found that there is an exponential relationship between $\lambda_{\text {eff }}$ and the Darcy velocity. The evaluation is done for different points in time and the effective conductivity increases with time. Thus, the duration of a thermal response test under subsurface flow conditions can be expected to have an influence on the estimated thermal conductivity of the subsurface, even if the line source condition for the required test time is fulfilled. This result goes together with comparable examinations in the literature, e.g. a study done by Chiasson et al. [2000], who uses a compilation of typical hydraulic and thermal properties of soils and rocks for his preliminary studies. More recently, Huber [2013] also found an exponential relationship between $\lambda_{e f f}$ and the filter velocity in laboratory, field and numerical examinations.

\subsubsection{Discussion}

A series of parametric variations is performed to study the impact of different internal and external BHE factors and conditions on the reaction and efficiency of a Double- $U$ BHE. 
The results from the thermal conductivity studies in Figure 4.16 and Table 4.2 are important for the further development of BHE components. Due to the results, an improvement of the grout material would provide most benefit to reduce the joint thermal resistance, but also thermally enhanced heat pipes would be rewarding. The insight into the nearly reciprocal relationship between the components and $R_{b}$ can help to enhance the further development of BHE models that are based on serial resistance networks.

It could be shown that the efficiency of this BHE type is optimised when the pipe distance is at its maximum, confirming the results from other studies in the literature. It is also found that there is an optimal pipe radius that is clearly larger than the reference value from the test site setup. Further investigations according the pipe thickness optimizsation are recommended because of the potential BHE improvement. Additionally, the energy consumption of the water pumps would also be reduced by larger pipe radii due to a smaller pressure drop.

The groundwater flow studies confirm recent results from numerical and experimental investigations. The influence of subsurface flow on the performance of BHEs is quantified for the reference setup. It turned out that the results of a state of the art TRT evaluation may lead to high deviations of the estimated thermal conductivity from its real value. Even moderate groundwater filter velocities lead to a heavy overestimation of $\lambda_{\text {eff }}$. This error in the TRT evaluation is not directly a problem for the dimensioning of a single BHE if the groundwater flow conditions are stable. However, in the case of unsteady conditions (e.g. if the subsurface flow was mainly caused by a heavy rainfall event during the TRT), the calculated borehole depth due to the test result may be underestimated. 


\section{Chapter 5}

\section{Oscillatory Thermal Response Tests}

\subsection{Background}

One of the central issues in shallow and also deep geothermics is the uncertainty concerning the subsurface properties at any desired potential production site. Also, there are uncertainties about the borehole itself and its back-filling quality. The approaches to examining these unknown factors are as old as the geothermal production technique itself; for decades, there have been attempts to find ways to improve the investigation methods.

During the last twenty years, the TRT has become the state-of-the-art test in geothermics. It provides a reasonable estimation of the anticipated productivity and the required dimension of the borehole(s). Nevertheless, the expressiveness of the testing results is restricted to two quantities, the effective thermal conductivity $\lambda_{\text {eff }}$ and the borehole thermal resistance $R_{b}$.

The idea, presented in this chapter, is to extend the amount of information that is received from the one-dimensional time-dependent temperature signal of the BHE system response by using oscillatory injection rates instead of a constant step. The expected improvements of this approach are that (i) the investigation area can be explicitly controlled and limited, because the thermal signal penetration depth is limitable by adjusting the 
oscillation frequency, and (ii) there will be additional information about the borehole and subsurface properties, gained by the system response time. Another advantage is that oscillatory system excitations lead to periodic state conditions that can be measured and evaluated more precisely than transient signals.

From a long-term period point of view, the oscillatory heat extraction of BHEs has already been the topic of a number of studies. The annual heat load of a seasonal recharged borehole is approximately comparable to a sine function. The long-time effects of (unbalanced) seasonal heat loads is a recent topic of interest, e.g. studied by Lazzari and Zanchini [2012], Zanchini et al. [2012]. In this thesis, the focus is on the evaluation of the system responses due to oscillatory injected short period heat loads, which are limited to experimentally feasible time frames. The basic idea and parts of the results are published (Oberdorfer [2013]).

\section{Hot Wire Method}

Groot et al. [1974] and Healy et al. [1976] developed an instrument to measure the thermal conductivity of gases by using an instantly joule heated wire that is placed within the gas. They used the same analytical solution based on Kelvins line-source theory (Carslaw and Jaeger [1959]) as it is used for the evaluation of subsurface TRTs. In doing so, they utilised the fact that the electric resistance of a metal wire depends on its temperature. Thus, a constant electric power output leads to a temperature increase that can be quantified by the voltage if the current is kept constant. The calculation of the thermal parameters of the sample are analogous to the evaluation of a TRT within the validity of the demand that $a t / r^{2} \gg 1$. Sandberg et al. [1977] showed that it is also possible to determine the heat capacity of a liquid sample with this method. After determining $\lambda$ from the slope of $\log (t)$ in Equation 2.22, they solve for the thermal diffusivity $a$ and thus receive the volumetric heat capacity $\rho c_{p}$.

The transient method is based on the assumption that the sample is infinitely extended in space because boundary effects are not considered in the line source solution. Thus, the sample that is to be examined has to be big enough so that its boundary temperatures are not disturbed during the whole measurement period. Depending on the sample material's properties, this steady state method may cause the need for big material probes. One solution for this problem is to utilise periodic thermal waves instead of 
a constant heat load. The major advantage of this approach is that waves tend to decay because of diffusion. The depth of penetration is a function of the excitation frequency and the thermal properties of the probe. In case of an electric heated wire, the line source approximation can be applied to oscillatory heating rates. Griesinger et al. [1997] extended the $3 \omega$ method $^{1}$ from Cahill and Pohl [1987] to the hot wire method. Due to their promising results, they conclude that the common hot wire method can be extended with low effort by applying oscillatory signals instead of constant heating. This extension decreases the amount of necessary probe material and allows the determination of the thermal conductivity and diffusivity of the material probes. Additionally, Atalla et al. [1981] showed that the influence of convective processes in liquid probes becomes negligible when the penetration depth of the thermal waves is small.

\section{Hydraulic Analogy}

Oscillatory system excitations are a topic of recent interest in the branch of hydrogeology. The one dimensional representation of Equation 2.14

$$
\rho c_{p} \frac{\partial T}{\partial t}=\lambda\left(\frac{1}{r} \frac{\partial^{2}}{\partial r^{2}}\right) T
$$

for constant thermal conductivity is identical to the transient equation that describes the hydraulic head $h$ that changes due to well pumping in a non-leaky confined aquifer,

$$
S \frac{\partial h}{\partial t}=K\left(\frac{1}{r} \frac{\partial^{2}}{\partial r^{2}}\right) h
$$

with the hydraulic conductivity $K$ and storage parameter $S$. This mathematical and physical analogy was already utilised in the 1930s by Theis [1935] who derived the hydraulic solution due to the heat equation line source solution from Carslaw [1906]. The similarities and limitations of both applications, hydraulic pumping tests and thermal response tests, are discussed e.g. by Eppelbaum and Kutasov [2006]. An early example for oscillatory pumping is the work of Kuo [1972] who applied this technique to petroleum reservoirs. Recently, Cardiff et al. [2013a,b] combined a steady-periodic 2D-model for oscillatory aquifer flow with a state-based sensitivity analysis and derived sensitivity

\footnotetext{
${ }^{1}$ The name describes the method: A $3 \omega$ harmonic appears, when a wire that is heated by a current of period $\omega$ dissipates at a frequency of $2 \omega$ and the voltage, that results from multiplication of the current $(\omega)$ and the electric resistance $(2 \omega)$ is measured.
} 
maps for source - receiver adjustments. They concluded that high-frequency oscillations tend to be more sensitive to "near field" parameters whereas low-frequencies draw an altogether more diffuse picture of the inhomogeneities.

The most important difference between the thermal and hydraulic approaches is the fact that pressure waves in the subsurface reach a certain penetration depth at much higher frequencies, i.e. short period lengths, compared to thermal waves. Thus, an oscillatory pump test can be performed within minutes or hours while an oscillatory thermal test would take weeks or months under the same conditions. For this reason, oscillatory thermal response tests are from the start either

- extremely time consuming, with timescales of weeks or months, if a more voluminous area is to be investigated with this method

or

- limited to get information about the BHE, the borehole and its very nearby ambient, if the testing time is limited to e.g. the typical duration of common TRTs.

If the focus of interest of an oscillatory investigation is the extended geological environment, one could e.g. utilise the system responses due to seasonal injections and evaluate them using the analytical solutions (Equation 5.5 and Equation 5.6, or analog solutions for different injection functions). If the BHE itself is to be analysed, one rather needs to excite the system at higher frequencies and utilise numerical analysis to interpret the thermal responses. The latter approach is the scope of the experimental and numerical analysis of the problem below.

\subsection{Oscillatory Heat Injection in BHEs}

Under certain conditions and approximations, the theoretical considerations of the oscillatory heated electric cable can be applied to oscillatory heated BHEs. The periodic heated line source at long time periods $t_{0}$ is a basic case that even provides an analytical solution. It can be obtained by setting the heat injection per unit length $q(t)$ in Equation 2.18 to

$$
q(t)=q_{p} \cdot \sin \left(\omega_{p} t\right), \quad \omega_{p}=2 \pi / t_{0} .
$$


The response functions of an oscillatory stimulated system are of the form

$$
T_{p, b}=-q_{p} \cdot R_{p} \cdot \sin \left(\omega_{p} t-2 \pi \Phi_{p}\right),^{2}
$$

if the excited system is LTI (Linear Time Invariant). A sufficient condition for a system to be LTI is that responses due to oscillatory excitations are damped and phase-shifted, but they will always have the same frequency because of the system linearity (Jackson [1991]). Thus, any LTI system could also be described by the Laplace transform of its impulse response or transfer function.

$R_{p}$ denotes the thermal resistance of the periodically heated (and/or cooled) system and $\Phi_{p}$ quantifies the phase shift of the thermal response. If the heat source can be simplified to a heated line, there exists an analytically approximate solution in analogy to the constant heated line (Equation 2.22). Eskilson [1987, paper 1, chap. 8] derived expressions for $R_{p}$ and $\Phi_{p}$ due to the solution from Carslaw and Jaeger [1959, p. 263]. The results are the complex-valued Kelvin functions

$$
R_{p}\left(r_{p b}\right)=\frac{1}{2 \pi \lambda} \cdot \sqrt{\left(\log \left(2 / r_{p b}\right)-\gamma\right)^{2}+\pi^{2} / 16}
$$

and

$$
\Phi_{p}\left(r_{p b}\right)=\frac{1}{2 \pi} \cdot \operatorname{atan}\left(\frac{\pi / 4}{\log \left(2 / r_{p b}\right)-\gamma}\right)
$$

with the Euler-Mascheroni constant $\gamma=0.5772 \ldots$ and the argument

$$
r_{p b}=r_{b} \sqrt{2} / d_{p}
$$

where

$$
d_{p}=\sqrt{a t_{0} / \pi}=\sqrt{2 a / \omega_{p}}
$$

is a measure of the thermal signal penetration depth. In correspondence to the constant heated line source approximation, which is only sufficient for large times $t \geq 5 r_{b}^{2}$, this solution is only valid for long periods $t_{0}$. Eskilson recommended $r_{p b}<0.1$ as a criterion, which may easily lead to minimal period lengths of up to several months ${ }^{3}$. The analytical solution is thus only suitable for heat injection/extraction oscillations on the seasonal

\footnotetext{
${ }^{2}$ Note that $T_{p, b}$ denotes the borehole temperature and not the resulting pipe fluid temperature that is evaluated in usual TRT applications.

${ }^{3} \mathrm{~A}$ moderate thermal diffusivity of $a=1 e-6\left[\mathrm{~m}^{2} / \mathrm{s}\right], r_{b}=0.1[\mathrm{~m}]$ leads to a minimal period length of $t_{0} \approx 72[d]$ to fulfil the criterion.
} 


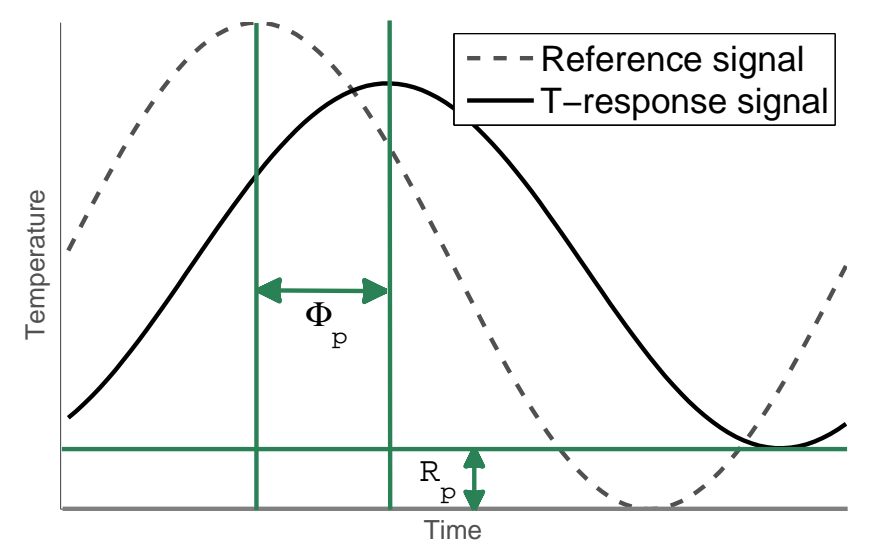

Figure 5.1: Phase gap and amplitude damping of the temperature response due to oscillatory excitation of any desired LTI system

timescale, but not for examinations that are to be performed during hours or days. The deviation between this solution and the system response due to highly frequent excitations is discussed below.

\subsection{Experimental Set Up}

The Geo-Solar-WP project test site facility at the ISFH ground in Hameln was used to perform an oscillatory thermal response test in July / August 2013. The experiment is determined to take place at the end of the project time and as the last long-time experiment, because of the expected heavy thermal disturbance of the subsurface that it would cause.

Due to the comprehensive measurement and control equipment at the test site, as described in Section 3.1, it is possible to perform any desired time-dependent heat injection rate and to $\log$ the resulting fluid temperatures and depth-resolved temperature reactions. The experimental heat injection rate is supposed to be a sinusoidal function with the amplitude $P_{1}$ and the angular frequency $\omega_{0}$. Yet, such a sinusoidal signal would be very hard to realise due to the technical restrictions of the heat pump, which would have to switch between cooling and heating modes smoothly and quickly. Thus, a constant heat rate $P_{0}$, which is larger than the oscillation amplitude, is added to the sinusoidal signal:

$$
P(t)=P_{0}+P_{1} \cdot \sin \left(\omega_{p} t\right), \quad\left|P_{0}\right|>\left|P_{1}\right|
$$



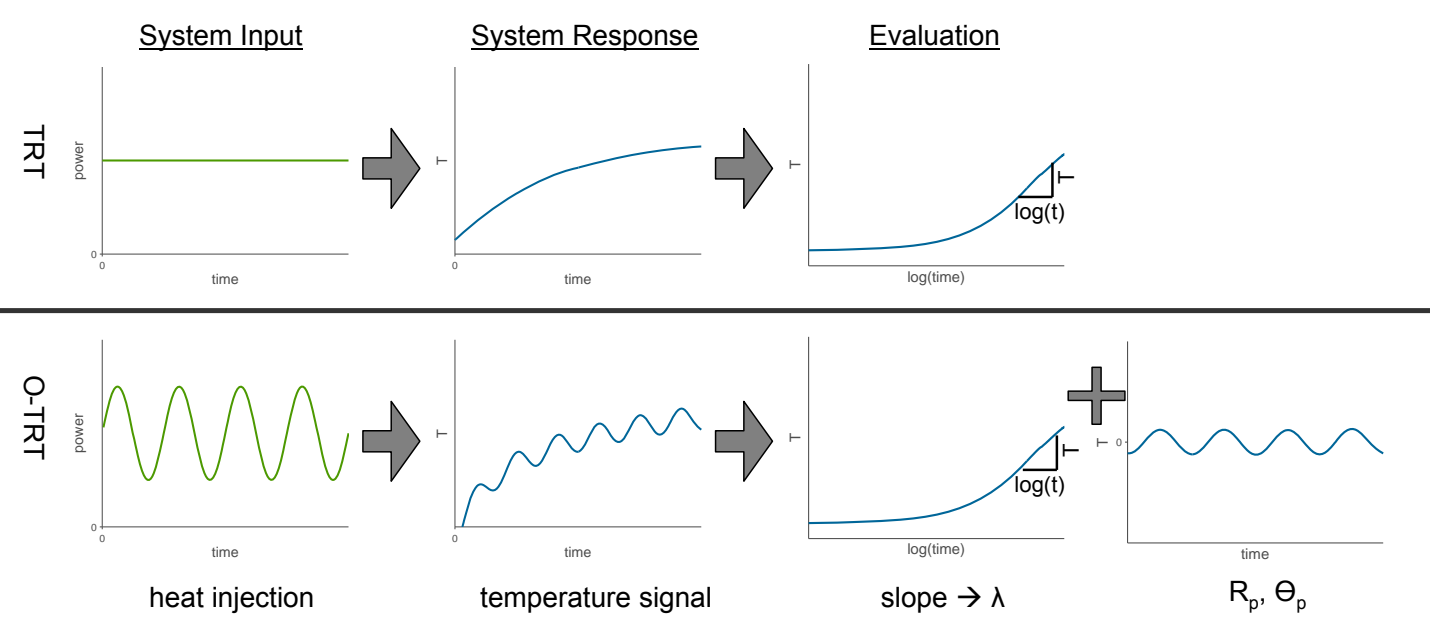

FIgURE 5.2: Comparison of the TRT and Oscillatory TRT principles

Now only a positive heat load, i.e. heat injection, is left over and the extraction parts vanish because the sign of $P(t)$ is always positive. This kind of signal is experimentally realised by an electric heating device, which is technically easier and more precise to control than a heat pump. The advantage of overlapping of the two injection rates is that the thermal response signals can be separated again in the post-processing routines. This offers the possibility of also evaluating the constant heat injection response part, which is actually a classical TRT evaluation. The principle of the O-TRT is shown in comparison to the state-of-the-art TRT in Figure 5.2.

Figure 5.3 shows the heat injection rate for the complete test. The excitation frequency $\omega_{0}$ is changed six times during the test, leading to seven different periods. It is derivable from the theory of heat conduction, see e.g. Carslaw and Jaeger [1959], that the resulting temperature, caused by two independent heat source functions, can be expressed as a superposition of the resulting temperatures due to the single sources. It follows that the temperature development of the BHE becomes the superposition of a conventional TRT with the constant heating rate $P_{0}$ and an oscillatory response caused by the heating rate $P_{1} \cdot \sin \left(\omega_{p} t\right)$.

The injection rate amplitudes of the three BHEs are not equal, as shown in Figure 5.3. This is due to the fact that the power control is realised integrally and not individually, i.e. the heat injection rate is controlled due to the resulting mixing temperature of all three BHEs. From this it follows that the effective injection rate in each BHE depends on its thermal contact to the borehole and the subsurface. The amplitude is significantly 


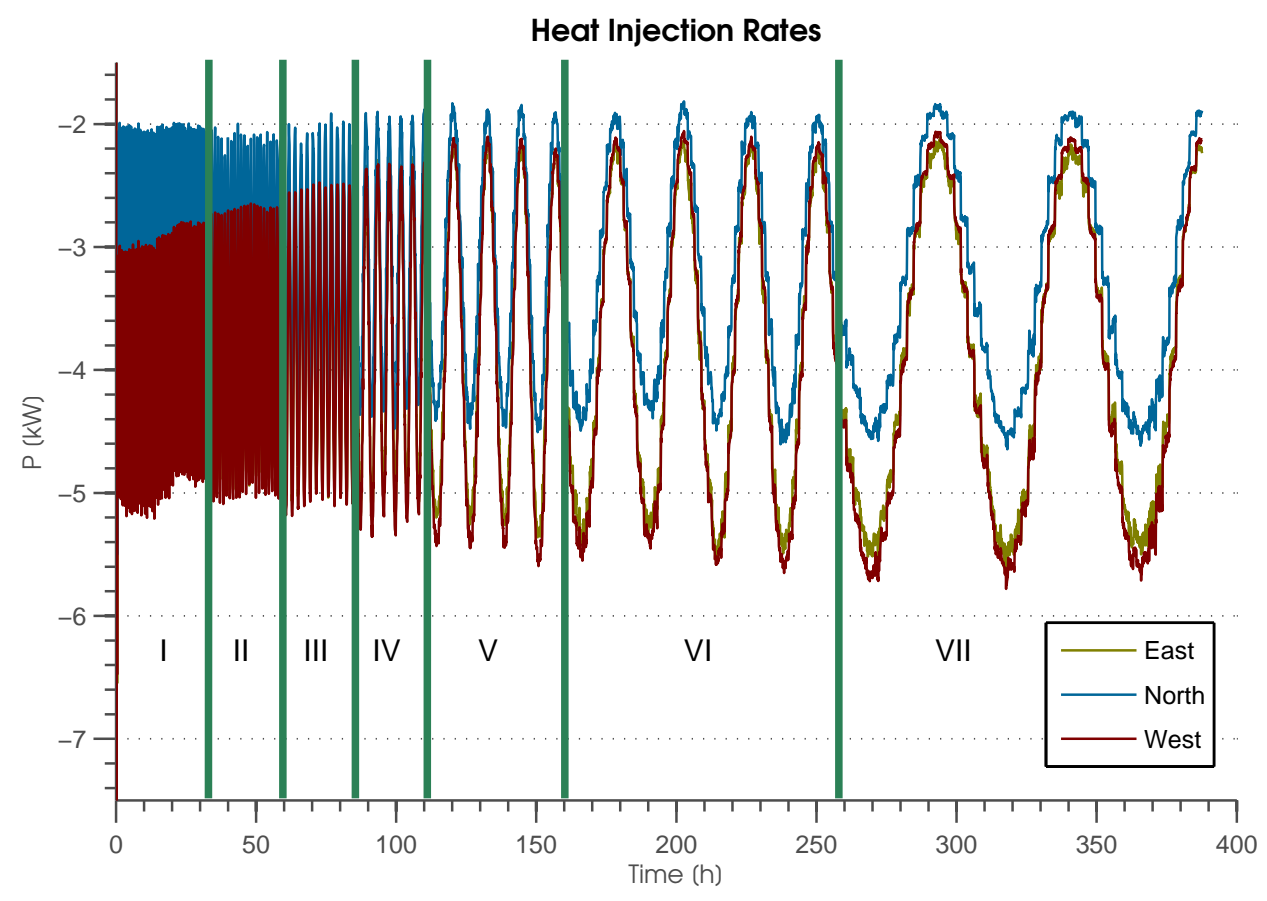

FiguRE 5.3: Heat injection rates in the three test site BHEs (SE, SN, SW) during the O-TRT. I-VII mark periods with different excitation frequencies.

lower at the northern BHE (SN) which comes up with the lowest value of $\lambda_{\text {eff }}$ and the highest borehole thermal resistance $R_{b}$, as shown in Table 3.2. The experimental resulting temperatures are plotted in Figure 5.4. As expected, the inlet temperatures of all three BHEs are similar, but the outlet temperature of SN deviates visibly.

The technical advantage of the overlapping constant and oscillatory injection is, as mentioned before, that no heat pump is needed because no cooling is exerted. Due to this technical simplification, the oscillatory test can be arranged with conventional TRT equipment, only a time-discrete control of the heating rate is necessary. However, due to technical restrictions, the oscillatory signal had to be approximated by discretisation into $n=25$ constant heat steps per period. Although the resulting temperatures are smoothed due to diffusive processes within the pipe fluid, the influence of the discrete stepping method is apparent especially for low frequencies, which can be seen on the right side of Figure 5.3.

\subsubsection{Results}

The experimental data base gained from the in situ oscillatory experiments at the ISFH test site in Hameln is very comprehensive. It contains a wide set of values including 


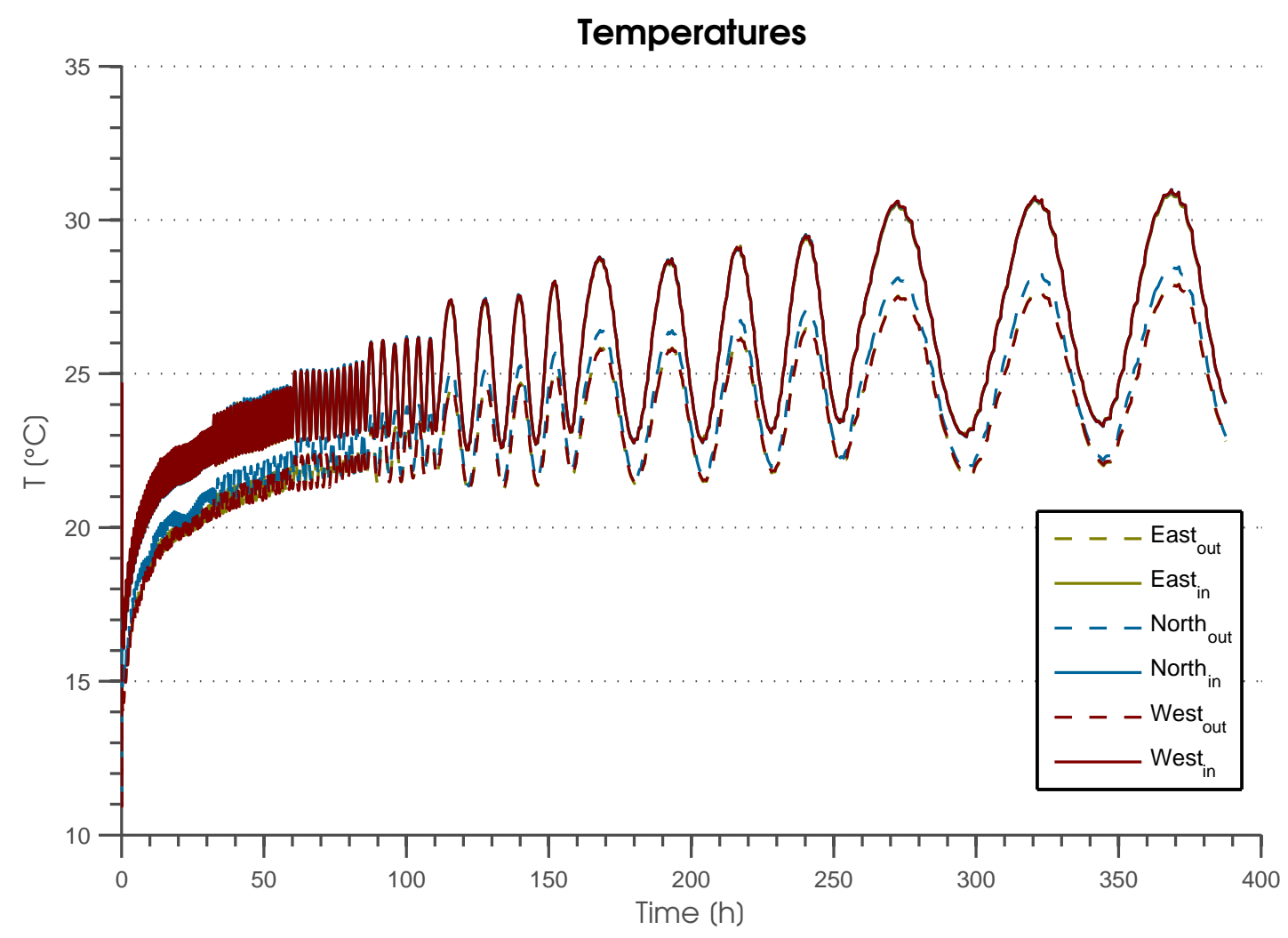

FIGURE 5.4: In- and outflow temperatures during the O-TRT

temperatures, heat fluxes and flow rates at a sampling rate of 30 seconds. The duration of the oscillatory test of more than two weeks thus led to nearly 50,000 discrete data sets.

The mean fluid temperature is determined as the arithmetic mean temperature between the BHE in- and outflow temperatures. For the evaluation of the oscillatory part of the heat injection, the corresponding temperature signals need to be separated into one part that represents the constant heat injection response and into another part resulting from the oscillation, as illustrated in Figure 5.2. This signal post-processing is done using the commercial software MATLAB. In a nutshell ${ }^{4}$, the oscillatory parts of the temperature signals (Figure 5.4) are identified by finding the local extremes and calculating their mean value. The resulting mean temperature curve represents the constant heat injection component of the signal. If this component is then subtracted from the raw signal, only the oscillatory parts remain. The remaining oscillatory signal

\footnotetext{
${ }^{4} \mathrm{~A}$ more detailed description can be found in Appendix C
} 


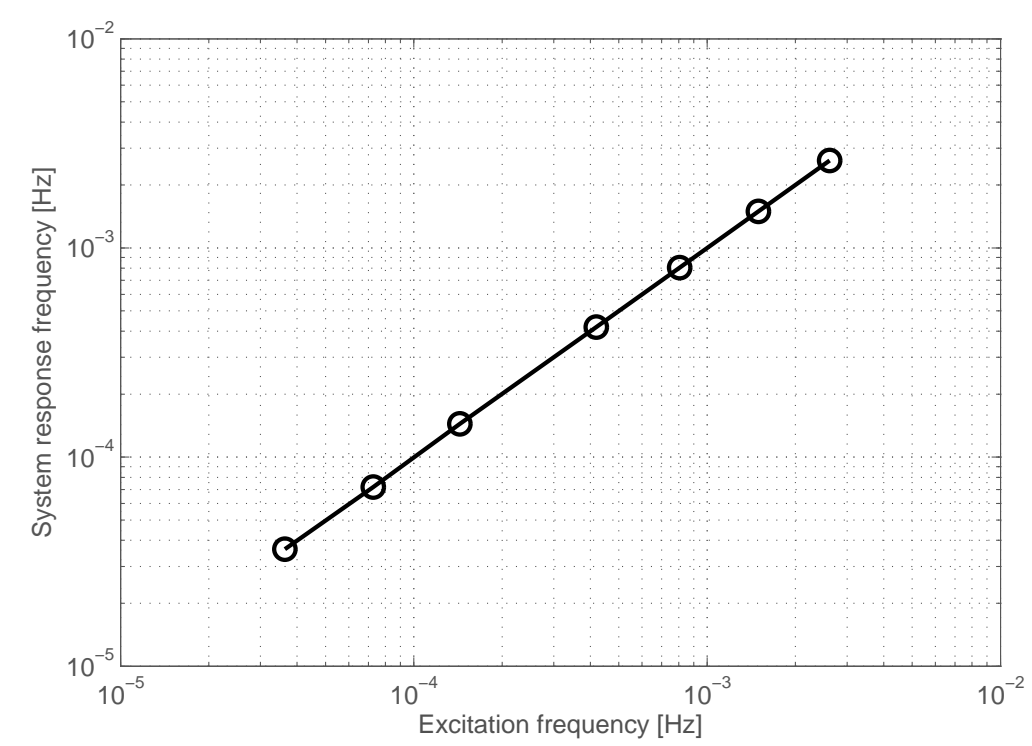

FiguRE 5.5: Excitation frequency vs. system response frequency of SE

is now fitted to a sine function

$$
f(t)=\beta_{1}+\beta_{2} \cdot \sin \left(\beta_{3} t+\beta_{4}\right)
$$

to gain the essential characteristic parameters $\beta_{i}$ of the oscillation. The amplitude $\beta_{2}$ of the sine function is converted into the oscillatory resistance $R_{p}$ using Equation 5.4

$$
R_{p}=\frac{\beta_{2}}{q_{p}}
$$

and the phase shift from the fitting function phase $\beta_{4}$ is analogously

$$
\Phi_{p}=-\frac{\beta_{4}}{2 \pi} .
$$

The results of the post-processing procedures for the different period lengths are summarised in Table 5.1.

A preliminary question of interest is whether the system is actually an LTI system. This is examined by comparing the excitation frequencies and the system response frequencies in Figure 5.5. Apparently, both frequencies are equal over a wide range of periods and it can be concluded that the presumption of linearity is accurate regarding the relevant frequency range.

The oscillatory resistance $R_{p}$ and phase shift $\Phi_{p}$ are plotted as functions of the period 
length in Figure 5.6 and Figure 5.7 . The system responses of the three examined BHEs on oscillative excitations are visibly disparate. For small period lengths, the oscillatory resistances are similar and they diverge for longer periods. Especially the northern BHE comes up with a remarkably high long-period resistance. Thereby, the phase shift shows the opposite behaviour; the northern BHE phase shift is significantly higher than the shift from the other two, but the difference decreases with increasing period length. Furthermore, the phase shifts between the eastern and western BHE differ, but only in the mid-frequency range.

\begin{tabular}{|c|c|c|c|c|c|}
\hline Period & $\begin{array}{c}t_{0} \\
{[\mathrm{~min}]}\end{array}$ & BHE & $\begin{array}{c}q_{p} \\
{\left[W m^{-1}\right]}\end{array}$ & $\begin{array}{c}R_{p} \\
{\left[m K W^{-1}\right]}\end{array}$ & $\begin{array}{c}\Phi_{p} \\
{[\mathrm{rad}]}\end{array}$ \\
\hline \multirow{3}{*}{ I } & \multirow{3}{*}{40} & East & 14.83 & $2.46 \mathrm{E}-02$ & $1.10 \mathrm{E}-01$ \\
\hline & & North & 15.48 & $2.55 \mathrm{E}-02$ & $1.37 \mathrm{E}-01$ \\
\hline & & West & 15.14 & $2.46 \mathrm{E}-02$ & $1.09 \mathrm{E}-01$ \\
\hline \multirow{3}{*}{ II } & \multirow{3}{*}{70} & East & 16.20 & $3.30 \mathrm{E}-02$ & $1.10 \mathrm{E}-01$ \\
\hline & & North & 15.60 & $3.74 \mathrm{E}-02$ & $1.36 \mathrm{E}-01$ \\
\hline & & West & 16.46 & $3.29 \mathrm{E}-02$ & $1.07 \mathrm{E}-01$ \\
\hline \multirow{3}{*}{ III } & \multirow{3}{*}{130} & East & 17.94 & $4.38 \mathrm{E}-02$ & $1.09 \mathrm{E}-01$ \\
\hline & & North & 16.45 & $5.21 \mathrm{E}-02$ & $1.29 \mathrm{E}-01$ \\
\hline & & West & 18.25 & $4.34 \mathrm{E}-02$ & $1.05 \mathrm{E}-01$ \\
\hline \multirow{3}{*}{ IV } & \multirow{3}{*}{250} & East & 19.77 & $6.00 \mathrm{E}-02$ & $1.09 \mathrm{E}-01$ \\
\hline & & North & 16.99 & $7.48 \mathrm{E}-02$ & $1.26 \mathrm{E}-01$ \\
\hline & & West & 20.55 & $5.78 \mathrm{E}-02$ & $1.01 \mathrm{E}-01$ \\
\hline \multirow{3}{*}{ V } & \multirow{3}{*}{720} & East & 21.31 & $9.51 \mathrm{E}-02$ & $9.71 \mathrm{E}-02$ \\
\hline & & North & 17.66 & $1.21 \mathrm{E}-01$ & $1.04 \mathrm{E}-01$ \\
\hline & & West & 22.90 & $8.81 \mathrm{E}-02$ & $9.31 \mathrm{E}-02$ \\
\hline \multirow{3}{*}{ VI } & \multirow{3}{*}{1440} & East & 22.37 & $1.17 \mathrm{E}-01$ & $7.69 \mathrm{E}-02$ \\
\hline & & North & 17.65 & $1.54 \mathrm{E}-01$ & $7.95 \mathrm{E}-02$ \\
\hline & & West & 23.69 & $1.10 \mathrm{E}-01$ & $8.02 \mathrm{E}-02$ \\
\hline \multirow{3}{*}{ VII } & \multirow{3}{*}{2880} & East & 22.72 & $1.44 \mathrm{E}-01$ & $6.75 \mathrm{E}-02$ \\
\hline & & North & 18.44 & $1.84 \mathrm{E}-01$ & $5.81 \mathrm{E}-02$ \\
\hline & & West & 24.40 & $1.34 \mathrm{E}-01$ & $6.53 \mathrm{E}-02$ \\
\hline
\end{tabular}

TABLE 5.1: Post-processing results of the combined oscillatory TRT

During the O-TRT experiment, the depth-resolved temperature signals from the installed pt100 sensors were also logged. The evaluation of this data is shown in Figure 5.8. Note

\footnotetext{
${ }^{5}$ This kind of representation is similar to a Bode plot. Hendrik Wade Bode (1905-1982) derived a simple method for graphing the gain and phase-shift plots of a system's frequency response. The differences are that (i) the $\mathrm{x}$-axis here denotes the period length and not the frequency and (ii) the $\mathrm{y}$-axis in Bode plots represents the magnitude $[d B]$.
} 


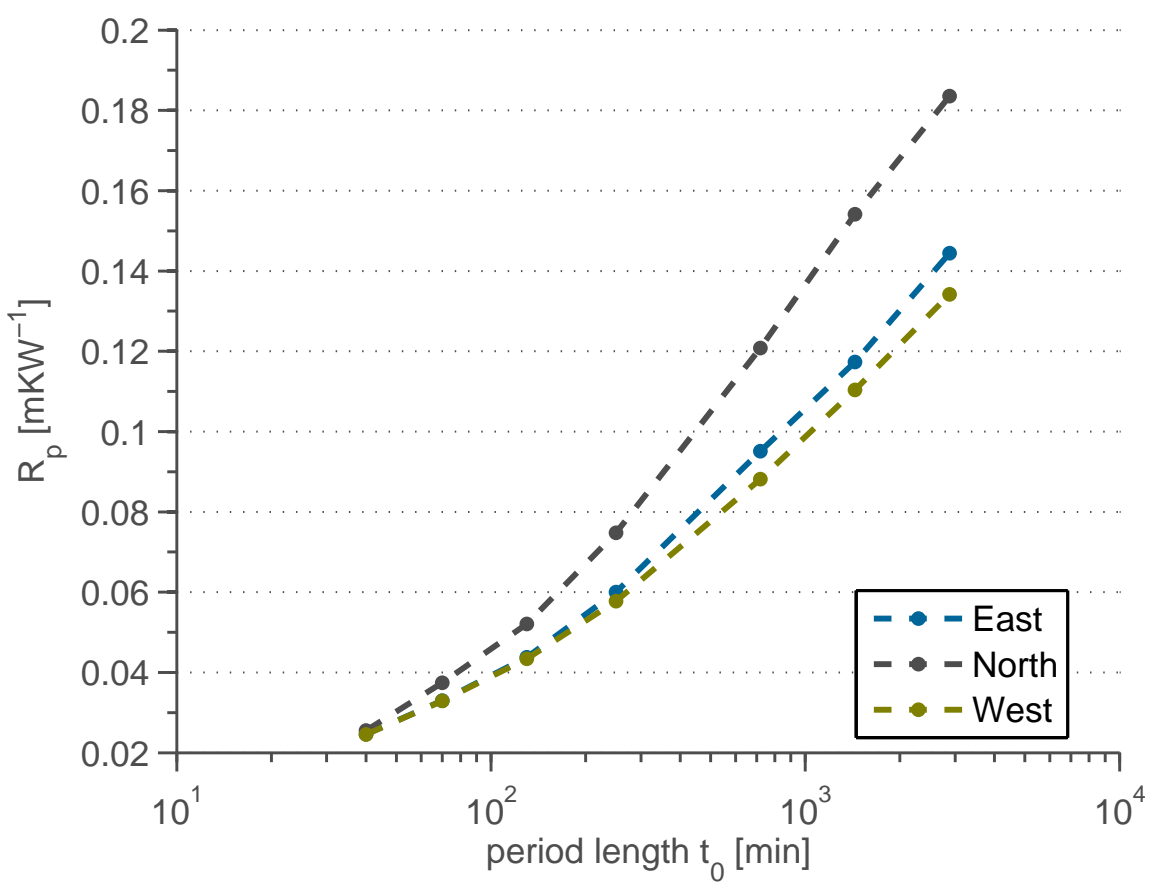

FiguRE 5.6: Oscillatory resistances of the experiment BHEs for different excitation period lengths (amplitude plot)

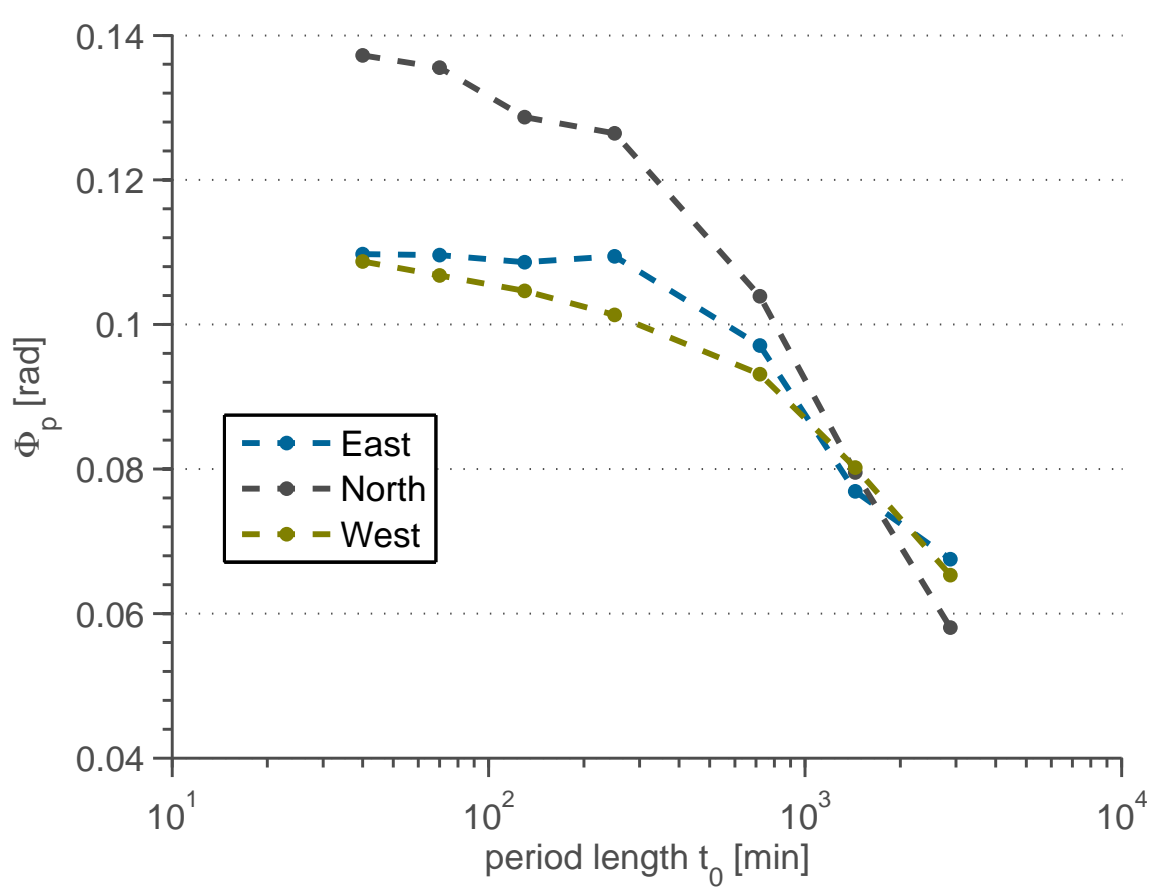

FiguRE 5.7: Phase shifts of the experiment BHEs for different excitation period lengths (phase plot) 
that the resulting amplitudes are not expressed in terms of the resistance. The reason for this is that the depth-resolved heat injection rate amplitude $q_{p}(z)$ is unknown ${ }^{6}$. Thus, the temperature values from the sensors are evaluated directly and not the oscillatory resistance, which could only be gained by dividing by $q_{p}(z)$.

Some signals of the wetern BHE $(21[\mathrm{~m}]$ depth for all period lengths and $49[\mathrm{~m}]$ depth for the three short period lengths signals) could not be evaluated due to a oversized noise-to-signal ratio.

The trends of the temperature curves are in all cases not very distinctive at high frequencies, but they develop their shape with increasing period length. Again, the northern BHE shows a different behaviour compared to the other ones. The temperature amplitude in the upper half region of the borehole is more damped than below. Also, the phase shift $\Phi_{p}$ is clearly higher in the upper $20[\mathrm{~m}]$.

Altogether, the evaluation results from the oscillatory heat injection experiments are interesting as there are significant differences in the resistances and the phase shifts. The results from the TRT in Table 3.2 showed the differences between the effective thermal conductivities and the borehole thermal resistances. The O-TRT results suggest that the conditions of the northern BHE are somehow special. The high phase shift during the short-time reactions imply that the reason for the differences is located inside the borehole because the temperature signal does not reach out into the subsurface for the highest frequency with $t_{0}=70[\mathrm{~min}]$.

Altogether, a more detailed interpretation of the experimental O-TRT results is impossible without any knowledge about the principles of the thermal responses of BHEs due to oscillatory excitations. Since no suitable analytical solutions for the high-frequency problem exist, the fundamentals are studied by utilising the developed numerical model.

\subsection{Numerical Studies}

The developed numerical BHE model approach is used to study the principles of the signal responses due to oscillatory heat injections. This is done with the Double-U

\footnotetext{
${ }^{6}$ Also, the temperature sensors are located outside of the pipes. Not the fluid temperature is measured, but the temperature at the pipe's outer surface.
} 

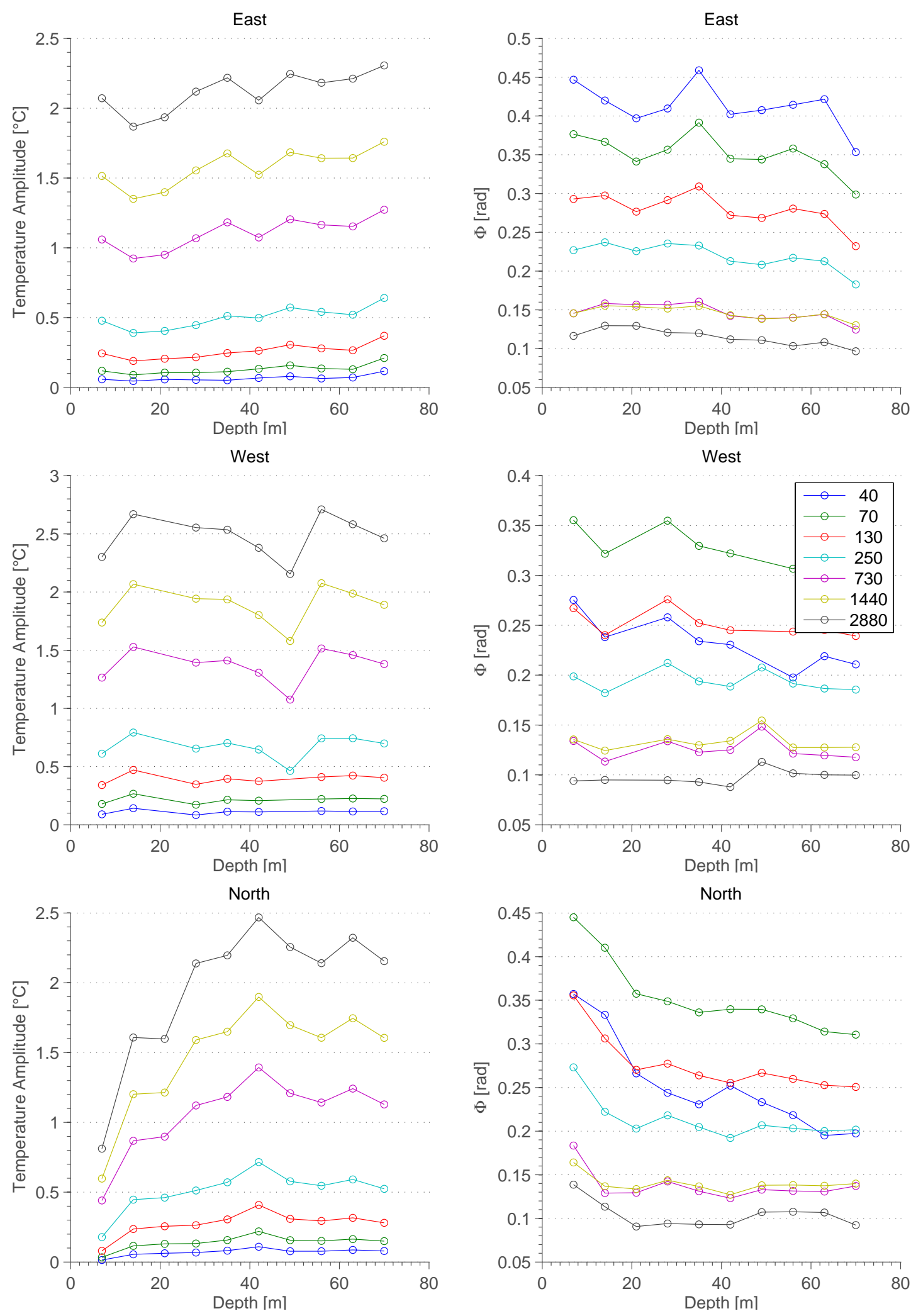

Figure 5.8: Experimental results of depth resolved temperature amplitudes and phase shifts of the three BHEs for different excitation periods $t_{0}[\mathrm{~min}]$ 


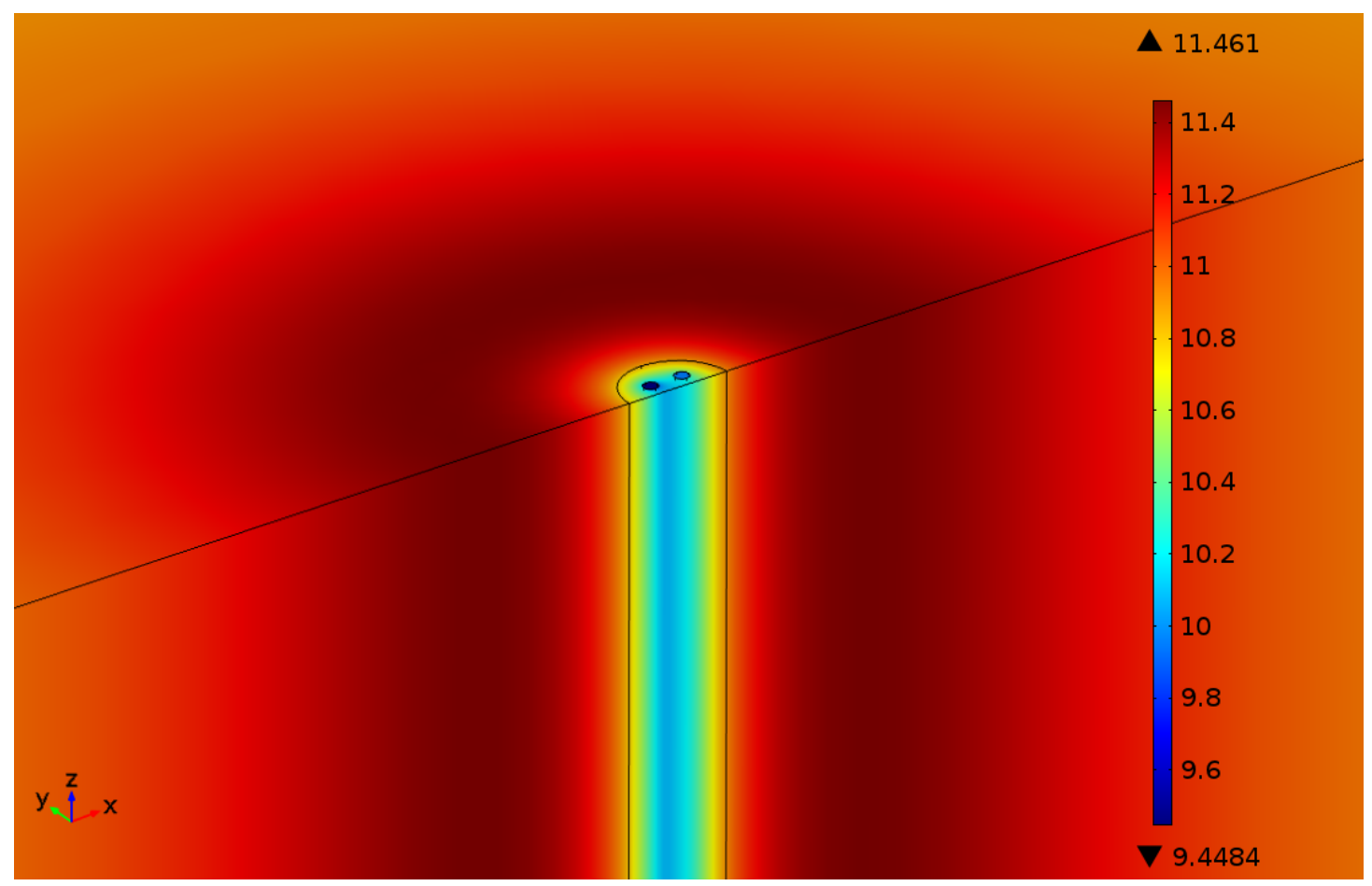

Figure 5.9: Temperature screen shot: Symmetry plane cut of a model run with an injection period length of $t_{0}=10[d]$ at the point in time $t=25[d]$, which is a zero crossing point of the oscillating heat injection rate. The colour bar shows the temperature $\left[{ }^{\circ} \mathrm{C}\right]$.

model that was calibrated with parameters from the ISFH testing site. Various parametric studies are performed to identify characteristic system reactions due to certain parametric changes of the model.

Figure 5.9 shows an exemplary screen shot of a $t_{0}=10[h]$ period length model run at a time point where the oscillative heat injection rate makes a zero crossing, flipping from heat injection to extraction; the radial spreading thermal wave is clearly seen. A detailed view of the radial temperature distribution at this point in time is to be seen in Figure 5.10, where this corresponding situation is plotted as profile in x-direction through the pipes in a depth of $1[m]$. This figure also points out the different pipe fluid temperatures from the down-flow (left) and the up-flow (right) branches. Here, the down-flow branch is of lower temperature, as suggested due to the onset of cooling at the heating rate sign change. 


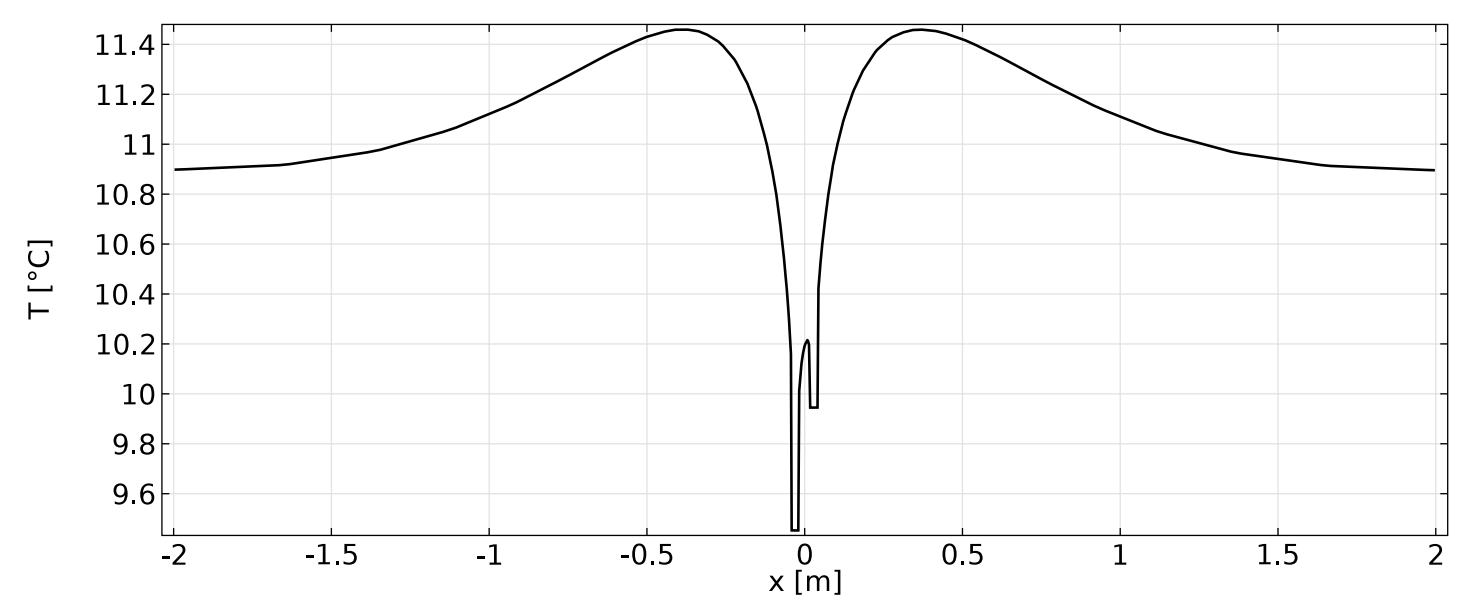

FiguRE 5.10: Horizontal temperature profile in x-direction through the down- and up-flow branches in a depth of $z=-1[\mathrm{~m}]$ at the same state as in Figure 5.9.

\subsubsection{Comparison to the Long-Period Analytical Solution}

The analytical solutions for the thermal resistance and phase shift of oscillatory heated line sources (Section 5.2) are only valid for long period lengths or low frequencies respectively. The numerical BHE model is used here to quantify the differences between the analytical line source approximation and the validated numerical approach. For this reason, both the solutions of both methods are compared at different system excitation frequencies. For the comparison, it is not the pipe fluid temperature response that is evaluated, but rather the mean borehole wall temperature because of the line source analogy. $\bar{T}_{w}(t)$ is numerically evaluated by integration over the whole borehole surface (and dividing by the surface area). The oscillation resistance $R_{p}$ and phase shift $\Phi_{p}$ are determined from this temperature, according to Equation 5.4.

The amplitude of the heat injection rate is $q_{p}=25\left[\mathrm{Wm}^{-1}\right]$ and the period length is varied between 1 and 200 days. The system response parameters are calculated due to a similar routine as is done with the experimental data. The model subsurface geometry radius has to be enlarged to $r_{s}=10[\mathrm{~m}]$ to prevent influences from the model outer boundary. The reason is that the long period lengths excitations of up to $200[d]$ cause a much longer signal penetration depth as occuring within standard TRT timescale, which is a number of hours up to some days.

The results of the resistances are shown in Figure 5.11 and the phase shifts are plotted Figure 5.12, respectively. Due to Eskilson [1987], the Kelvin series expansion that leads to the analytical solutions is valid for small quantities $r_{p b}<1$. The criterion lower limit 


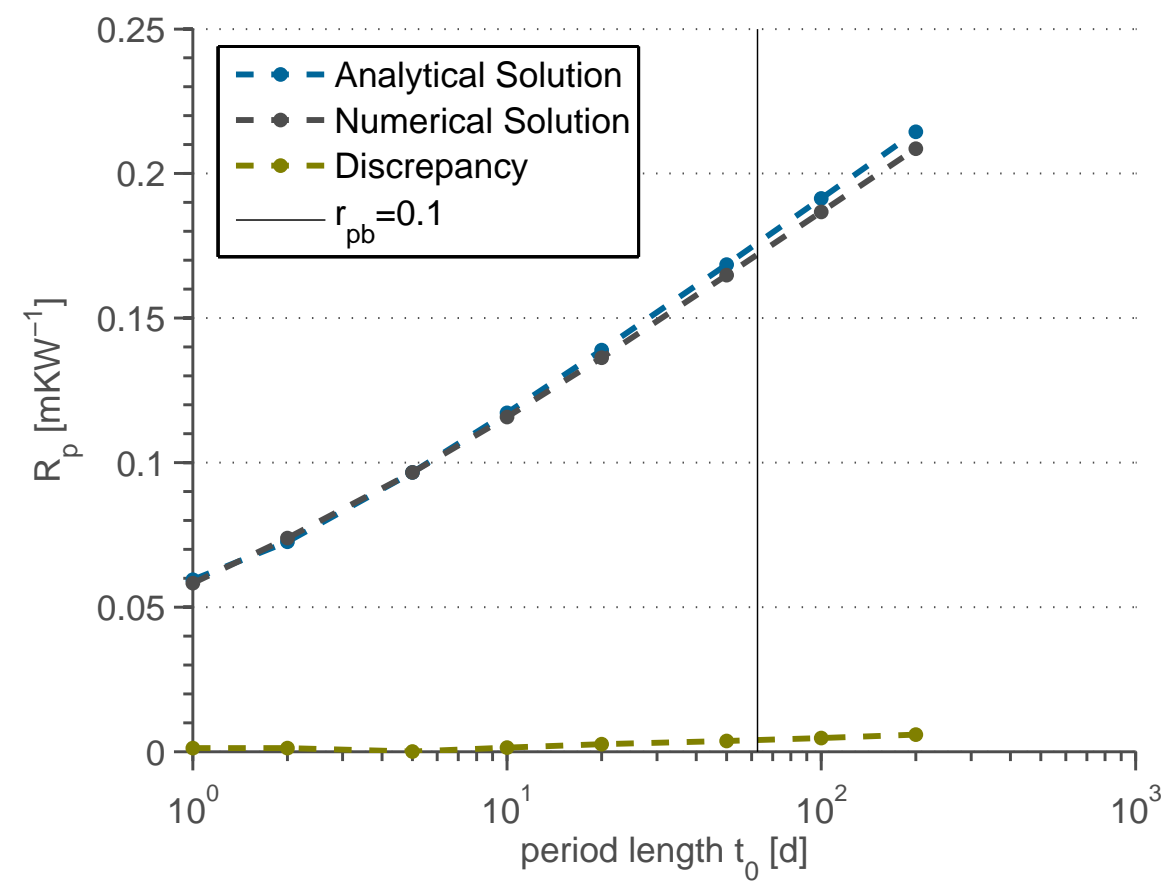

FIGURE 5.11: Comparison between analytical and numerical solutions of the oscillatory thermal resistance

$r_{p b}=1$ is also marked in the plots. Both studies are compared and the discrepancies between the temperatures are shown in both figures in green colour. The difference between the phase shifts of both solutions becomes significantly smaller for period lengths that are longer than the period length requested by the criterion. The difference between the resistances $R_{p}$ are small for all period lengths, and the graphs rather slightly diverge with increasing $t_{0}$. The reason for the onset of slight derivation is not clear, but possible explanations are (i) the increasing influence of the top and bottom boundaries of the cylindrical subsurface domain, that start playing a role for injection time frames in the order of several months or (ii) the increasing influence of temperature-dependent material properties that are considered by the numerical model but neglected by the analytical solution.

Apart from the small differences between the solutions, the curves of the numerical and the analytical solutions show the same characteristic behaviour that appears in the case of oscillatory heat injection by (line) sources: The oscillatory resistance is increasing with $t_{0}$ because this quantity includes the resistance of the whole thermal mass that is covered within the penetration depth of the signal, which is increasing with increasing period length. At the same time, $\Phi_{p}\left(t_{0}\right)$ is decreasing because the phase shift becomes less important compared to the increasing length of the periods. 


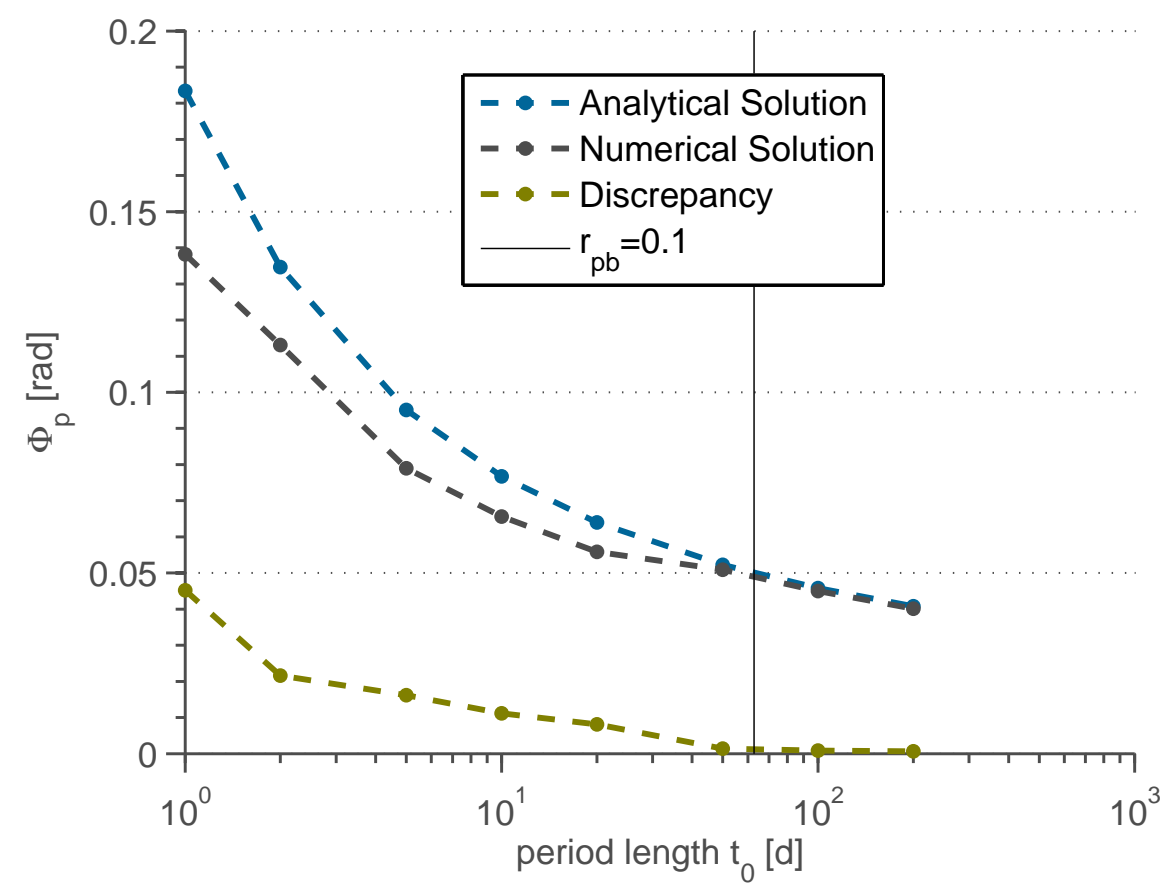

Figure 5.12: Comparison between analytical and numerical solutions of the signal phase shift

\subsubsection{System Response Studies}

As mentioned before, the numerical full 3D BHE approach constitutes a suitable tool for investigations of the O-TRT concept. Parametric studies are performed to examine the system responses due to a number of reasonable variations of BHE parameters and properties. The motivation is to utilise the conclusions to interpret the experimental results and to be able to make statements about the origin of certain system response effects. Similar to the experimental O-TRT, the results of the studies are presented in terms of the oscillatory resistance $R_{p}\left(t_{0}\right)$ and the phase shift $\Phi_{p}\left(t_{0}\right)$ from Figure 5.13 to Figure 5.19. The same MATLAB evaluation routine is used as for the experimental data evaluation; the oscillatory system responses are decomposed into their constant and oscillatory parts and the signal amplitudes and phase shifts are evaluated by a sine function fitting routine. The oscillatory heat injection rate per unit length of the BHE is here again $q_{p}=25\left[\mathrm{Wm}^{-1}\right]$. The scope of the following studies is the property of the BHE and of the subsurface ambient that underlies uncertainties at in-situ BHE installations. The aim is to obtain a set of reference data which can be used to classify the experimental results of the O-TRT approach. In doing so, similar period lengths as in the filed test are selected for the numerical study. 

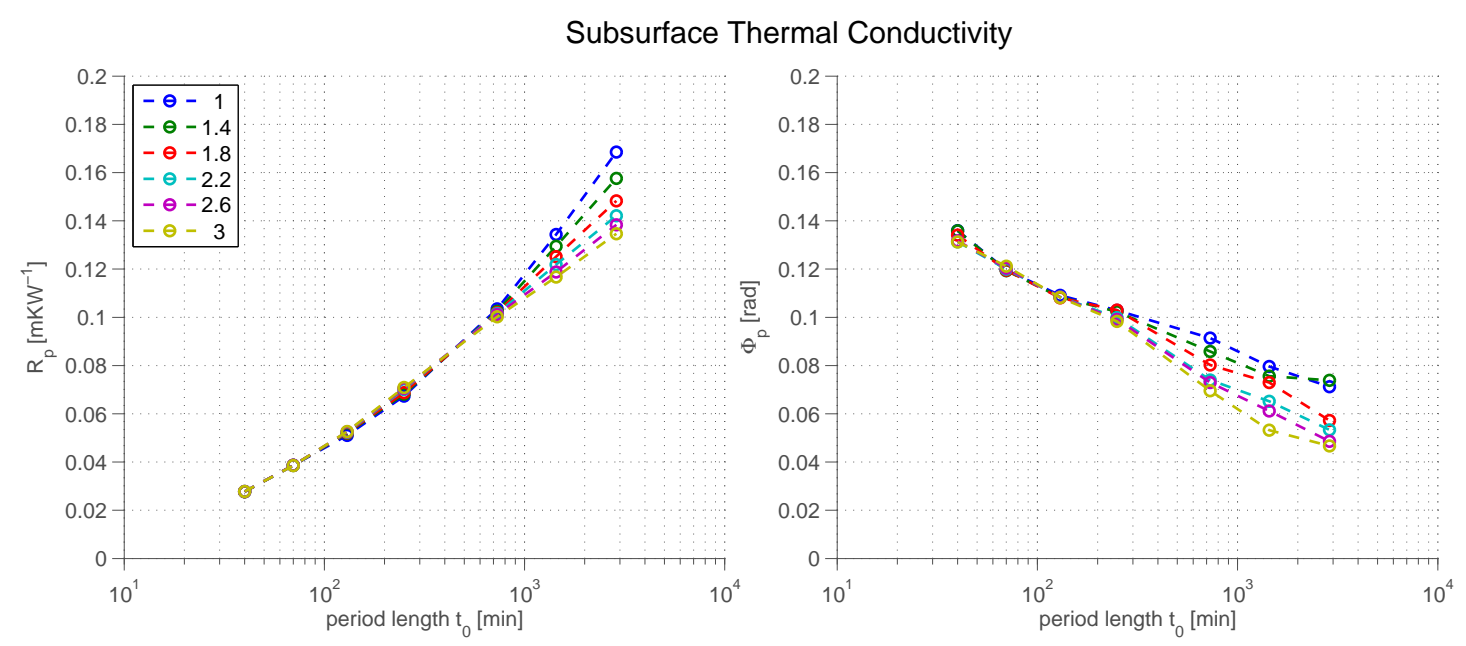

Figure 5.13: System responses of oscillatory heat injection. The thermal conductivity of the ambient subsurface is varied between $\lambda_{s}=1-3\left[\mathrm{Wm}^{-1} \mathrm{~K}^{-1}\right]$.

The first parameter to be discussed is the subsurface thermal conductivity which varies between 1 and $3\left[\mathrm{Wm}^{-1} \mathrm{~K}^{-1}\right]$, representing a reasonable range for typical in-situ arrangements. The results of this study are displayed in Figure 5.13. It follows that, for high frequency excitations, there is only a very small variation of both, $R_{p}$ and $\Phi_{p}$. The onset of deviations of the system responses due to subsurface property differences appears only for a period length of at least some hours. This is the time scale that is needed for the penetration depth to reach out of the borehole into the subsurface. For higher period lengths, the resistance increases with decreasing conductivity, but the phase shift is reduced. A similar result comes out for the subsurface specific heat capacity variation (Figure 5.14) for values between 800 and 2,800 $\left[\mathrm{Wm}^{-1} \mathrm{~K}^{-1}\right]$. The range of $c_{p}$ consciously surpasses the typical subsurface values of rocks and minerals because it represents the effective values, including different assumed groundwater contents. The deviations between the system responses due to the different excitation frequencies are less distinctive compared to the responses of the subsurface thermal conductivity variations.

The thermal properties of the borehole back-filling are varied in the same range as the subsurface values and the results are shown in Figure 5.15 and Figure 5.16. The most interesting contrast to the subsurface parameter variations is that the resistance differences also increase with increasing period length, but the phase shift shows the opposite behaviour: phase deviations between the different thermal conductivity results disappear for long period lengths. According to the grout heat capacity variation study, it seems like the arising deviation of $R_{p}$ also decreases for period lengths that are higher 

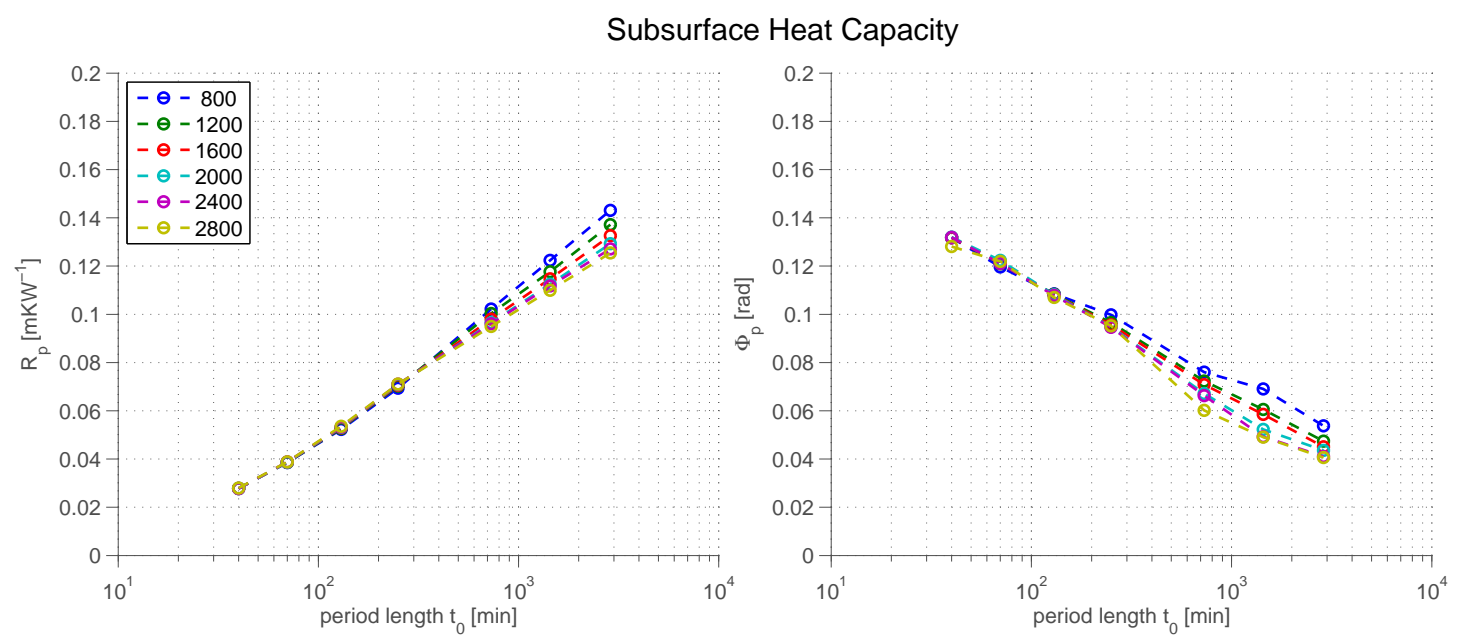

FIGURE 5.14: System responses of oscillatory heat injection. The heat capacity of the ambient subsurface is varied between $c_{p, s}=800-2800\left[\mathrm{Wm}^{-1} \mathrm{~K}^{-1}\right]$.
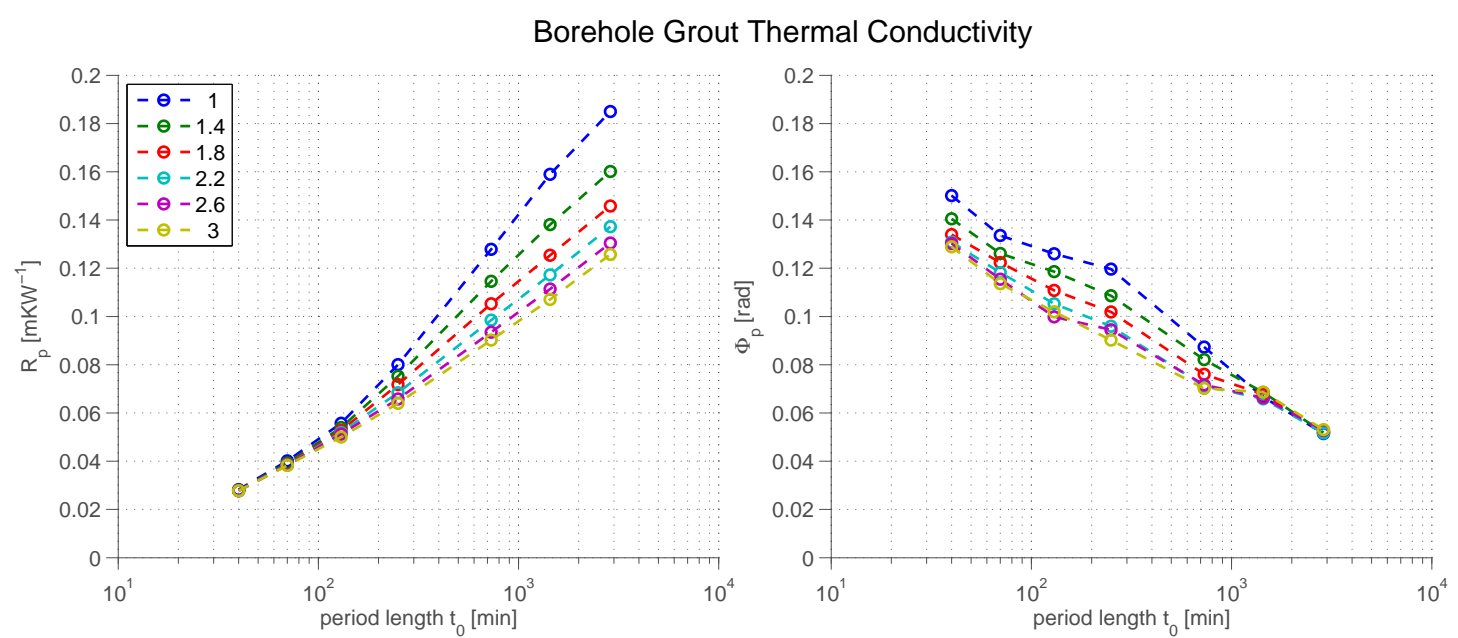

FiguRE 5.15: System responses of oscillatory heat injection. The thermal conductivity of the borehole grout filling material is varied between $\lambda_{g}=1-3\left[\mathrm{Wm}^{-1} \mathrm{~K}^{-1}\right]$.

than the study range, while the phase shift completely changes its shape and a saddle point appears for higher $c_{p}$-values.

An unknown factor concerning a back-filled BHE is the position of the pipes within the borehole. The influence of the pipe distance on the BHE performance is discussed above. Recent studies have shown that one cannot be aware of the exact positions ${ }^{7}$. Thus, two studies are performed where the distance between the down-flow and up-flow branches (Figure 5.17) and the distance between the two U-loops of the Double-U BHE (Figure 5.17) are varied between 3.66 and $12.81[\mathrm{~cm}]$, corresponding to 0.6-2.1 times

\footnotetext{
${ }^{7}$ Riegger [2011] removed the complete BHE including the grout back-filling after installation and saw it open across different sections. It was found that the positions of the pipes within in the back-filling were completely messed up, even though they placed spacers in $1[\mathrm{~m}]$ distance to each other.
} 

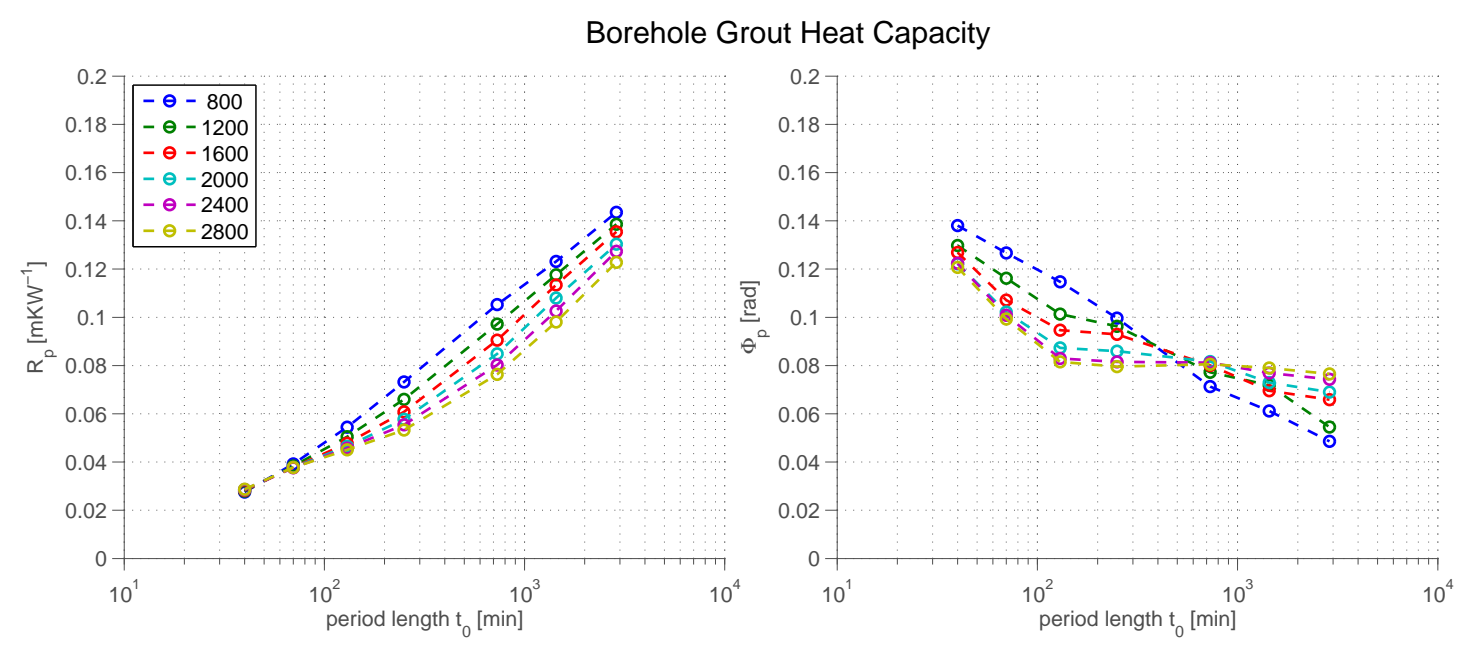

FIGURE 5.16: System responses of oscillatory heat injection. The heat capacity of the borehole grout filling material is varied between $c_{p, s}=800-2800\left[\mathrm{Wm}^{-1} \mathrm{~K}^{-1}\right]$.

the site-reference value of $6.1[\mathrm{~cm}]$. Apparently, both of the distance modifications lead to quite similar results. This is quite surprising because in the first case, the thermal short cut between the branches is affected, whereas in the second case alterations of the short cut do not directly appear if only the distance between the two loops is varied. The shapes of all four of the curves are somehow comparable to the grout heat capacity variations, besides the fact that the resistance curves do not seem to reunite for long period lengths but rather keep their distance. Also, the phase shift differences are not that distinctive here.

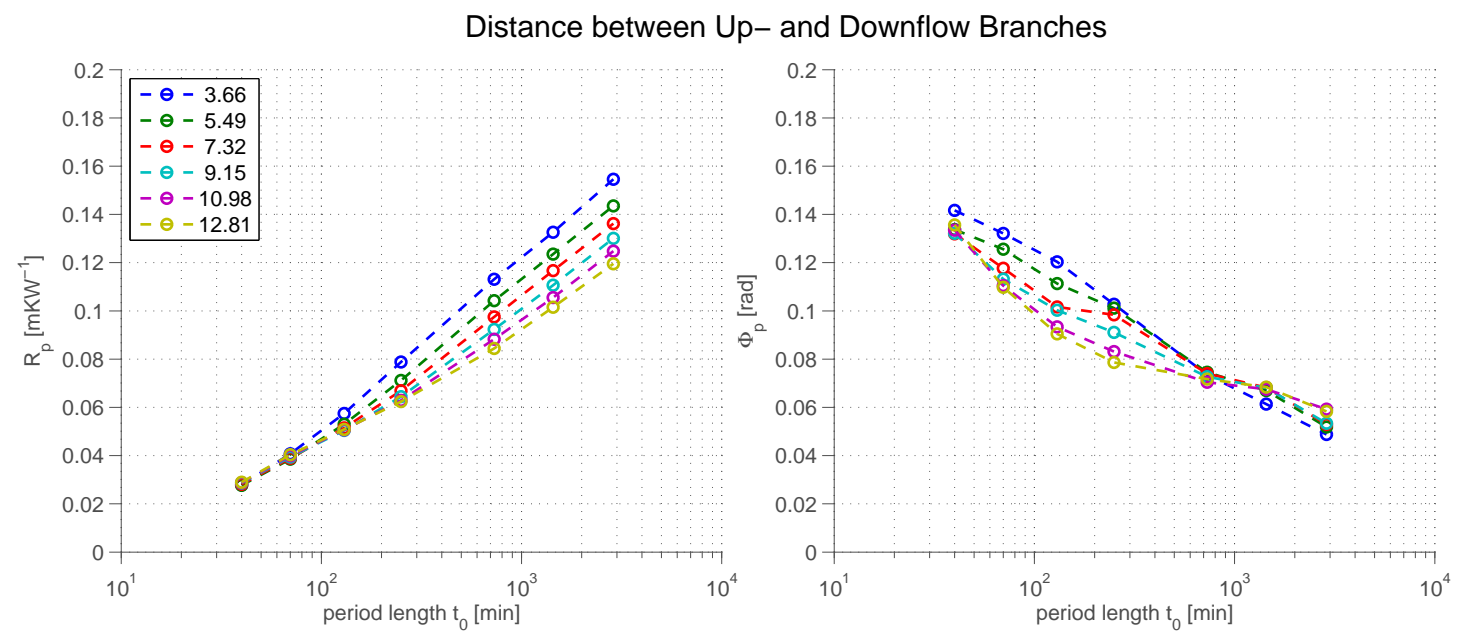

FIGURE 5.17: System responses of oscillatory heat injection. The distance between the up-flow and down-flow branches is varied between $d_{x}=36.6-128.1[\mathrm{~mm}]$ (i.e.

$$
\left.d_{x}=0.6 \cdot d_{x 0}-2.1 \cdot d_{x 0}\right) .
$$

Generally, ideal thermal contact between the borehole back-filling and the subsurface is 

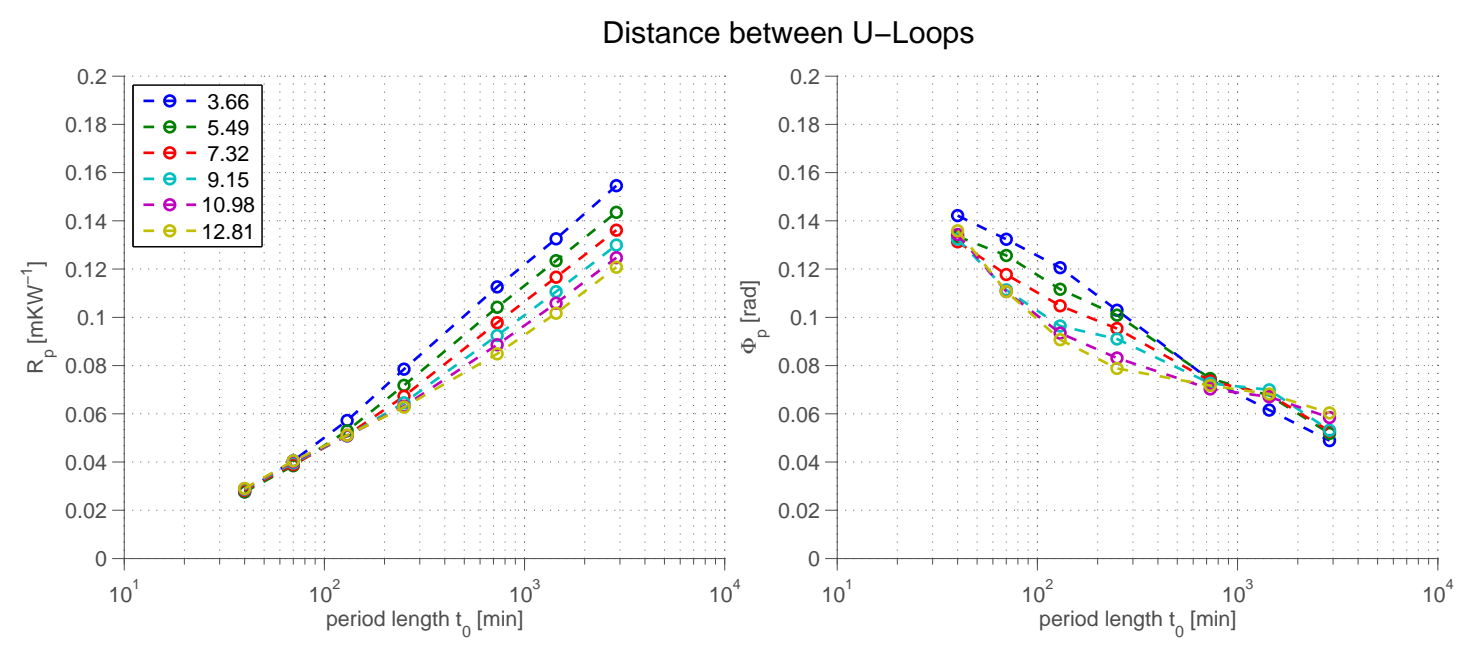

FiguRE 5.18: System responses of oscillatory heat injection. The distance between U-loops is varied between $d_{y}=36.6-128.1[\mathrm{~mm}]$ (i.e. $\left.d_{y}=0.6 \cdot d_{y 0}-2.1 \cdot d_{y 0}\right)$.

assumed in the numerical approach because of the lack of knowledge about the correct conditions. In an in-situ BHE, there may indeed be a resistance between these two domains, caused e.g. by a thin layer of air or water, leading to a retardation of the heat transfer process. The effect of the resistance layer is studied numerically here by applying an infinitesimally thin thermally resistive layer with a defined resistance to the model. The selected range is $R_{\text {layer }}=0.01-0.2\left[\mathrm{Km}^{2} \mathrm{~W}^{-1}\right]$, so the high values are in the range of the borehole's thermal resistance per surface area. The resulting system responses are shown in Figure 5.19. The oscillatory resistance is strongly increased for higher layer resistances, but again, the differences clearly do not set in before the penetration depth reaches the borehole wall. The onset of differences of the phase shift appears at a similar period length and the arising phase deviation is the most outstanding one compared to the other variation studies. However, it seems that the deviation between the curves decreases again for increasing period lengths.

\subsection{Discussion}

\section{Numerical Studies}

It could be shown that the system responses due to the oscillatory excitations of the numerical model converge against the analytical solution results. The deviation between the phase shifts nearly vanishes when the minimal-period-length criterion is reached, 

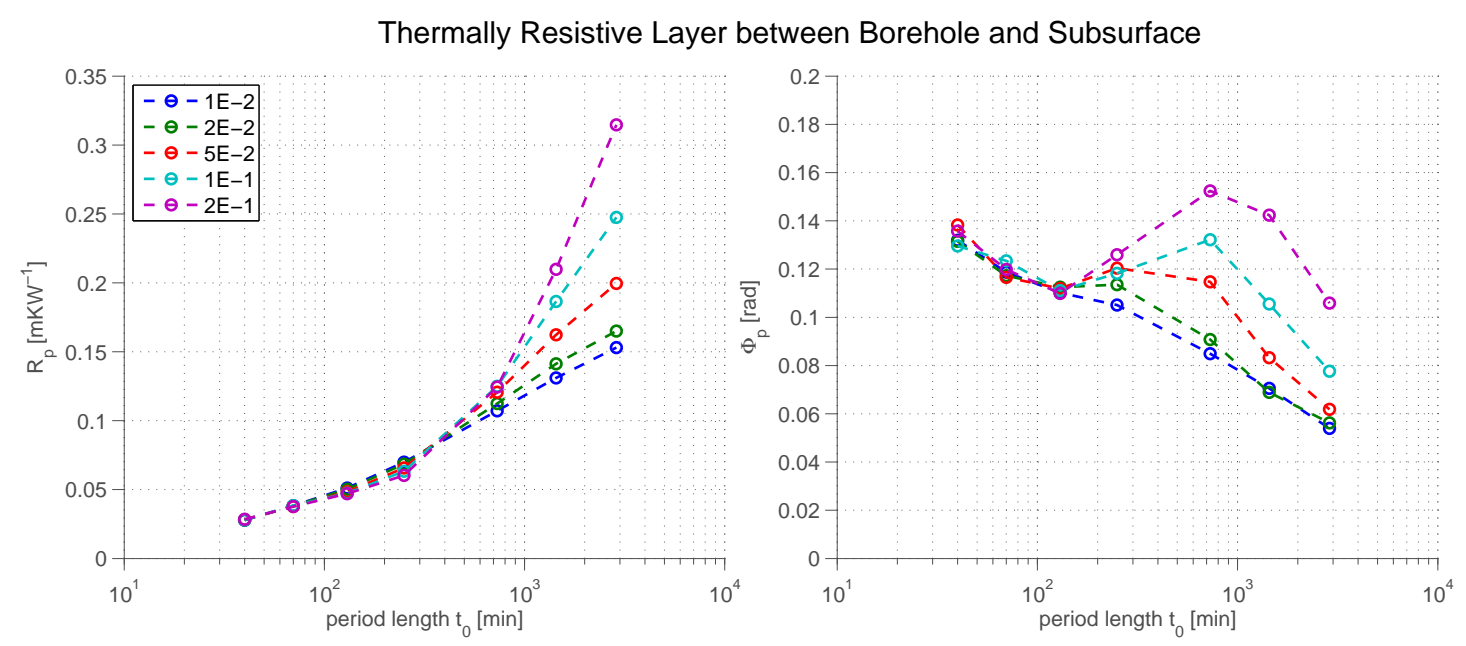

Figure 5.19: System responses of oscillatory heat injection. A thin thermally resistive layer is inserted between the BHE and the Subsurface domains and its resistance is varied between $R_{\text {layer }}=0.01-0.2\left[\mathrm{Km}^{2} \mathrm{~W}^{-1}\right]$.

whereas the oscillatory resistances already match fairly for short periods (their deviation rather increases slightly for longer period lengths).

The evaluation of the parameter studies of the O-TRT concept allows for general conclusions about the interpretation of experimental observations. It is found that

- one can in principle distinguish between borehole effects subsurface effects,

- effects from subsurface or borehole wall variations only appear at excitation period lengths, which are long enough so that the temperature signal reaches out of the borehole and

- phase shift deviations due to material conductivity variations, that are limited to the borehole region, disappear for long periods, whereas the oscillatory resistance deviations do not vanish.

\section{Interpretation of the Experimental Results}

The results from the experimental O-TRT at the test site lead to different system responses, although the BHEs are equally back-filled and installed very close to each other. It follows from the numerical study conclusions above that the high differences between the early time phase shifts do refer to borehole effects. As mentioned before, the BHEs were back-filled with different techniques. Regarding the numerical studies, the higher 
oscillatory resistance and phase shift of the northern BHE are probably due to the higher heat capacity and/or a lower thermal conductivity of the back-filling. Both can be explained by an increased water content of the borehole. The thermal conductivity of water is lower than the conductivity of the grout and it has a higher heat capacity. Since the water table is located at a depth of about 30 metres, it is possible that the back-filling in this BHE is disturbed by groundwater infiltration. The depth-resolved experimental results substantiate this explanation: The temperature amplitude is significantly increased in the lower borehole part. Probably, the filling method worked out better above the groundwater table, leading to a denser back-filling in the upper 30 metres.

Another noticeable aspect of the experimental results is the mid-period deviation between the eastern and western BHE phase shifts, which vanishes again for long periods (see Figure 5.7). Regarding the numerical studies, this effect matches with the thin resistive layer assumption results shown in Figure 5.19. Due to this interpretation, the deviation between the BHEs phase shifts can be explained by a quality difference between the thermal contact between the BHE and the subsurface. This is an example of a specific borehole issue which is not identifiable with the conventional TRT method, where all borehole effects accumulate in one quantity, $R_{b}$. 


\section{Chapter 6}

\section{Concluding Remarks}

The thermal use of the subsurface is supposed to become a more and more important part for the sustainable energies branch, and shallow geothermal heat exchangers are the fastest growing sector. For this thesis, a modelling concept of the heat transport phenomena within and around BHEs is elaborated. The model was successfully developed and validated. The results of the validation underline the high accuracy of the model, especially with regard to short-term system responses, which are often not sufficiently reproduced with other simulation approaches.

The model was then used to (i) investigate the improvement potentials of different BHE aspects and (ii) further develop the in situ methods to gain informations about the properties of the borehole-subsurface systems. The investigations concerning the parametric variations show that there is still potential to improve the performance of Double-U BHEs, e.g. the radius of the pipes could be optimised. A study about the influence of groundwater flow on the results of a TRT shows that advective transport may weight the estimation of the subsurface thermal conductivity gained by a TRT under moderate subsurface flow conditions.

A novel adaptive method to the state-of-the-art TRT with oscillating heat injection (O-TRT) could successfully be performed experimentally and studied numerically. An evaluation routine was developed that allows for the extraction of the characteristic system responses of BHEs due to oscillatory excitations. Systematic numerical studies point 
out the influences of different inherent properties on the system response signals, allowing for the classification of experimental O-TRT results into borehole and subsurface effects.

\subsection{Outlook}

In this work, a first approach of applying high-frequent oscillations to the TRT is presented. The promising results of the experimental tests together with the numerical studies are a first glance at this new method. The oscillatory heat injection concept should and will be further investigated. During the doctorate, the author took part in the planning and realisation phase of a new test site located on the property of the University of Göttingen. The site consists of wells that are $80[\mathrm{~m}]$ in depth and extensively equipped with depth-resolved temperature measurement instrumentation and the possibility to inject heat through copper wires. More details are described in Oberdorfer et al. [2013a]. The wells are arranged to a five-spot with very low horizontal distances of down to $1.5[\mathrm{~m}]$ and thus the site is appropriate for heat transport experiments and further investigations of the oscillatory approach. Due to the copper wires, even the analytical buried cable-solution can be applied instead of the more elaborate numerical approach.

Some of the results presented in this thesis pose new questions, e.g. why the deviation between the resulting resistivity of the numerical model and the analytical long-term solution slightly increases with increasing period lengths instead of vanishing, as the phase shift does. Further on, the influence of ground water flow, which is discussed for the classic TRT here, should be examined according to its impact on the oscillatory injections.

The presented numerical and experimental results of the O-TRT approach are gained using the Double-U BHE type. It would be interesting to examine whether the thermal responses of other designs fundamentally differ from the results in this thesis and if the the differences only appear within a certain range of excitation frequencies.

For the further development of the numerical BHE model regarding the O-TRT approach, it might be useful to solve the governing equations within the frequency-domain 
after Laplace transforming. This method would probably reduce the computational effort. 


\section{Appendix A}

\section{Nusselt Number Correlations}

In the literature, several approaches to determine $N u$ for transient and turbulent forced convection in pipes are used. Focusing on the simulation of geothermal BHE, there are some preferred correlations that are given below. In all cases, $N u_{0}$ is set to 4.36 for laminar flow that appears at $R e<2300$.

\section{Gnielinski Correlation}

The Gnielinski correlation for forced convection in pipes (Gnielinski [1976]) is

$$
\mathrm{Nu}=\frac{f D / 8 \cdot(\mathrm{Re}-1000) \cdot \operatorname{Pr}}{1+12.7 \cdot(f D / 8)^{1 / 2} \cdot\left(\operatorname{Pr}^{2 / 3}-1\right)}
$$

It is limited to $2300<\operatorname{Re}<6 \cdot 10^{6}$ and $0.5<\operatorname{Pr}<2000$ and depends on the Darcy Friction Factor $f_{D}$ which can be determined in the full range of Re (laminar, transition and turbulent) using the Churchill equation

$$
f_{D}=8\left(\left(\frac{8}{\operatorname{Re}}\right)^{12}+(A+B)^{-1.5}\right)^{1 / 12}
$$

where

$$
A=\left(-2.457 \ln \left((7 / \operatorname{Re})^{0.9}+0.27(e /(2 r))\right)\right)^{16},
$$


with the surface roughness $e$ and

$$
B=\left(\frac{37530}{\operatorname{Re}}\right)^{16}
$$

Gnielinski's correlation is e.g. used by Acuña [2010], Al-Khoury and Diersch [2011].

\section{Dittus-Boelter Correlation}

The most widely used correlation (see e.g. Chiasson et al. [2007], Rees and He [2013]), possibly because of its simplicity, is the Dittus-Boelter equation (Dittus and Boelter [1930])

$$
\mathrm{Nu}=0.023 \cdot \operatorname{Re}^{0.8} \cdot \operatorname{Pr}^{n}
$$

with $n=0.4$ for heating $\left(T_{\text {wall }}>T_{\text {fluid }}\right)$ and $n=0.33$ for cooling $\left(T_{\text {wall }}<T_{\text {fluid }}\right)$. The accuracy of the Dittus-Boelter correlation is anticipated to be $\pm 15 \%$ in the range of $10000<\operatorname{Re}<120000$ and $0.7<\operatorname{Pr}<120$ (Kutateladze and Borishanskii [1966]). Note that it is actually not valid in the transient flow zone.

\section{Petukhov Formula}

Merker [1987] uses the Petukhov formula for the turbulent regime at $10^{4}<\operatorname{Re}<5 \cdot 10^{6}$ and $0.5<\operatorname{Pr}<2000$ :

$$
\mathrm{Nu}_{\text {turb }}=\frac{\xi / 8}{K_{1}+K_{2} \sqrt{\xi / 8} \cdot\left(\operatorname{Pr}^{2 / 3}-1\right)} \operatorname{RePr}
$$

with

$$
K_{1}=1+27.2(\xi / 8)
$$




$$
K_{2}=11.7+1.8 \operatorname{Pr}^{-1 / 3}
$$

which seems to be related to Gnielinski's correlation, but falls back to a different friction factor:

$$
\xi=\frac{1}{(1.82 \log \operatorname{Re}-1.64)^{2}}
$$

Due to the lack of information about the transient regime at $2300<\operatorname{Re}<10000$, Huber and Schuler [1997] introduced a continuity approach that connects the laminar and turbulent fields:

$$
\mathrm{Nu}_{\text {trans }}=\mathrm{Nu}_{0, \exp }\left(\ln \frac{\mathrm{Nu}_{t u r b, \mathrm{Re}=1 e 4}}{\mathrm{Nu}_{0}} \frac{\ln (\mathrm{Re} / 2300)}{\ln (10000 / 2300)}\right)
$$

\section{VDI Wärmeatlas 2006}

The continuity approach by Huber and Schuler [1997] for the transient flow is just a kludge and is upgraded in GVC-VDI [2006] by introducing

$$
\mathrm{Nu}_{\text {trans }}=(1-\gamma) \mathrm{Nu}_{0}+\gamma\left(\frac{(0.0308 / 8) \cdot 10^{4} \cdot \operatorname{Pr}}{1+12.7 \sqrt{0.0308 / 8}\left(\operatorname{Pr}^{2 / 3}-1\right)}\left[1+(2 \cdot r / l)^{2 / 3}\right]\right)
$$

with

$$
\gamma_{k}=\frac{\operatorname{Re}-2300}{10^{4}-2300}
$$

for the transition range $2300<\operatorname{Re}<10000$ and quite similar to A for the turbulent range $\operatorname{Re}>10000$

$$
\mathrm{Nu}_{t u r b}=\frac{(\xi / 8) \cdot \mathrm{Re} \cdot \mathrm{Pr}}{1+12.7 \sqrt{\xi / 8} \cdot\left(\operatorname{Pr}^{2 / 3}-1\right)}\left[1+(2 \cdot r / l)^{2 / 3}\right]
$$


where

$$
\xi=\left(1.8 \log _{10} \operatorname{Re}-1.5\right)^{-2} .
$$

This correlation is used e.g. by Bauer [2011], Diersch et al. [2010]. According to 2.3, it shows an average behaviour compared to the other correlations and is used in this thesis unless otherwise specified. 


\section{Appendix B}

\section{Temperature Dependence of the}

\section{Working Fluid}

In all the numerical BHE models presented in this work, the temperature dependencies of the physical parameters of the BHE working fluids are taken into account. Usually, the working fluid is water and the well known temperature functions are already implemented in the COMSOL program package. The three BHEs at the ISFH test site in Hameln operate with a mix of water $(66 \%)$ and antifreeze (Tyfocor ${ }^{\circledR}, 33 \%$ ). The temperature dependencies of the mix can be interpolated from tables that are accessible to the public (Tyfocor [2013]). Figures B.1, B.2, B.3 show the functions of pure water and mix fluid which are relevant in this study.
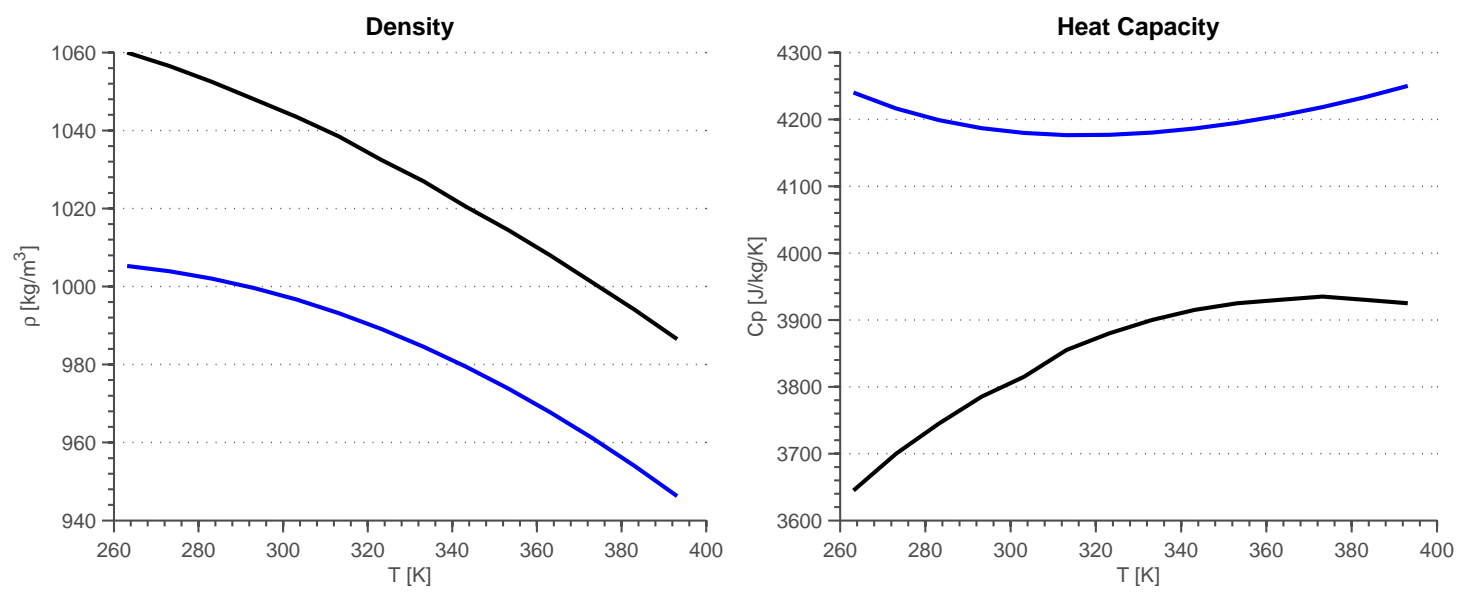

Figure B.1: Temperature dependencies of the density and heat capacity of water (blue) and water-antifreeze mix (black) 

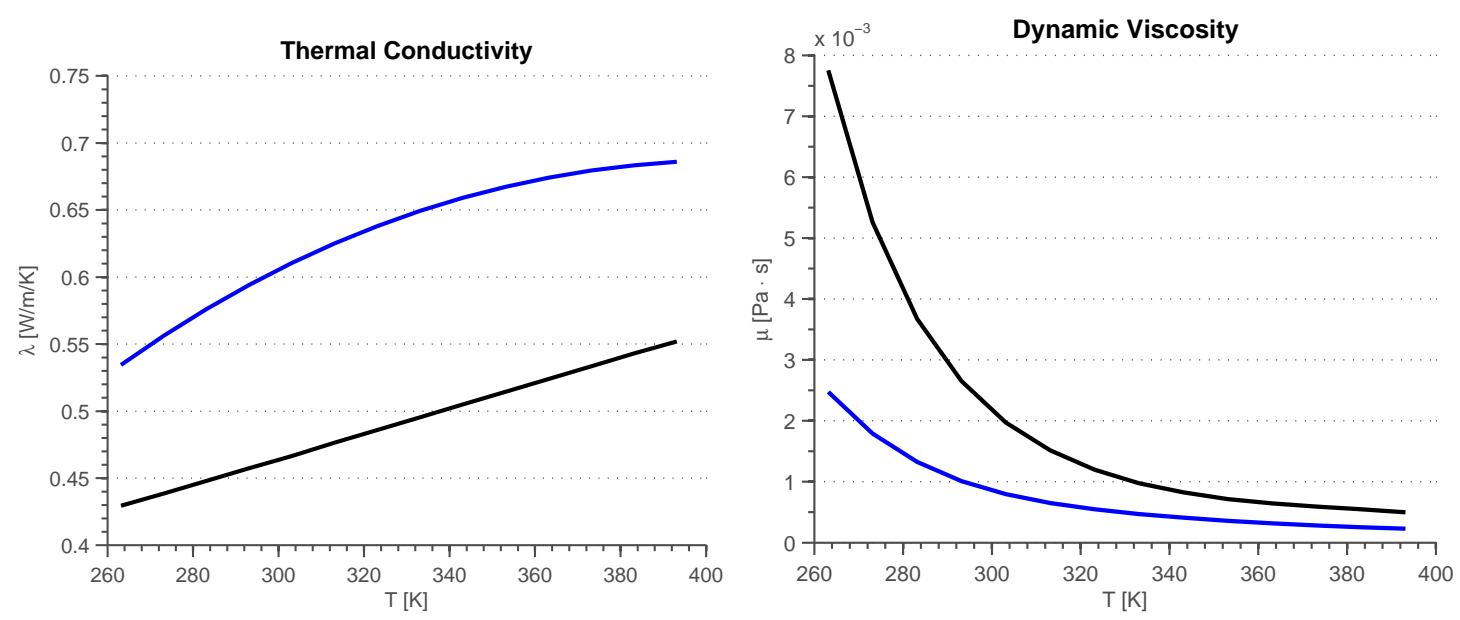

Figure B.2: Temperature dependencies of the thermal conductivity and dynamic viscosity of water (blue) and water-antifreeze mix (black)

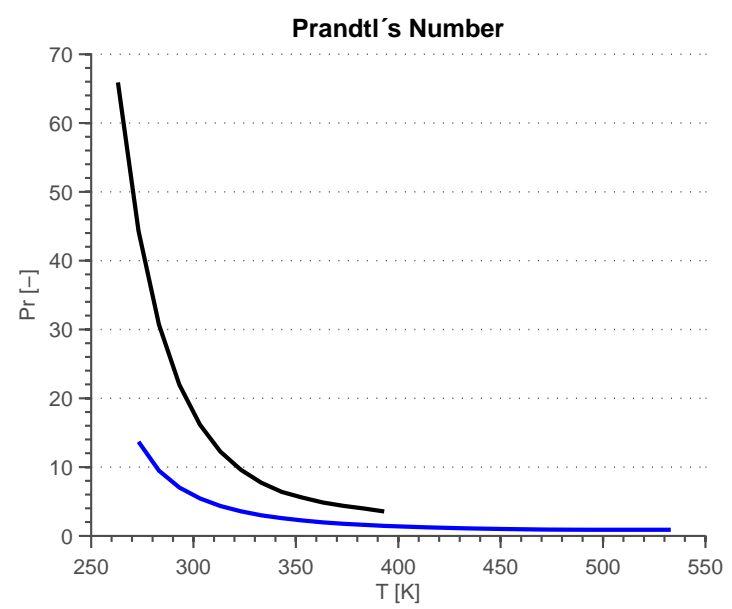

Figure B.3: Prandtl's Number of water (blue) and water-antifreeze mix (black) as functions of temperature 


\section{Appendix C}

\section{O-TRT Data Post-Processing}

The raw temperature data of the combined oscillatory and constant heat injection TRTs needs to be processed to separate the signals into oscillatory and constant parts. One problem is that the data sets are typically noisy. Therefore, a routine is needed that separates without changing the essential parameters of the oscillations. This is done in a self-written MATLAB program for time-dependent data processing. The program is divided into the following sequences:

1. Data Loading All necessary raw data sets are loaded into the workspace.

2. Filtering and Derivatives A digital filter (see Oppenheim et al. [1999]) is applied for smoothing the signal. The first order derivatives are calculated and again filtered. The next step is to find zero crossings of the derivatives. They give the points in time of the local extremes of the oscillations. The raw data at these times is evaluated to get the envelopes of the signal. Figure C.1 shows the raw data and envelopes for a part of the experimental period I signal $\left(t_{0}=40[\mathrm{~min}]\right)$.

3. Signal Separation The mean of the upper and lower envelope is calculated (blue line in Figure C.1. This is the part of the constant heating caused by the constant part of the heat injection rate, $P_{0}$. It is subtracted from the raw signal.

4. Sine Fitting The result from the subtraction is the oscillatory part of the temperature response, shown in Figure C.2. The raw data is fitted using a sine fitting procedure, a variation of the heuristic Nelder-Mead optimisation technique (Nelder 


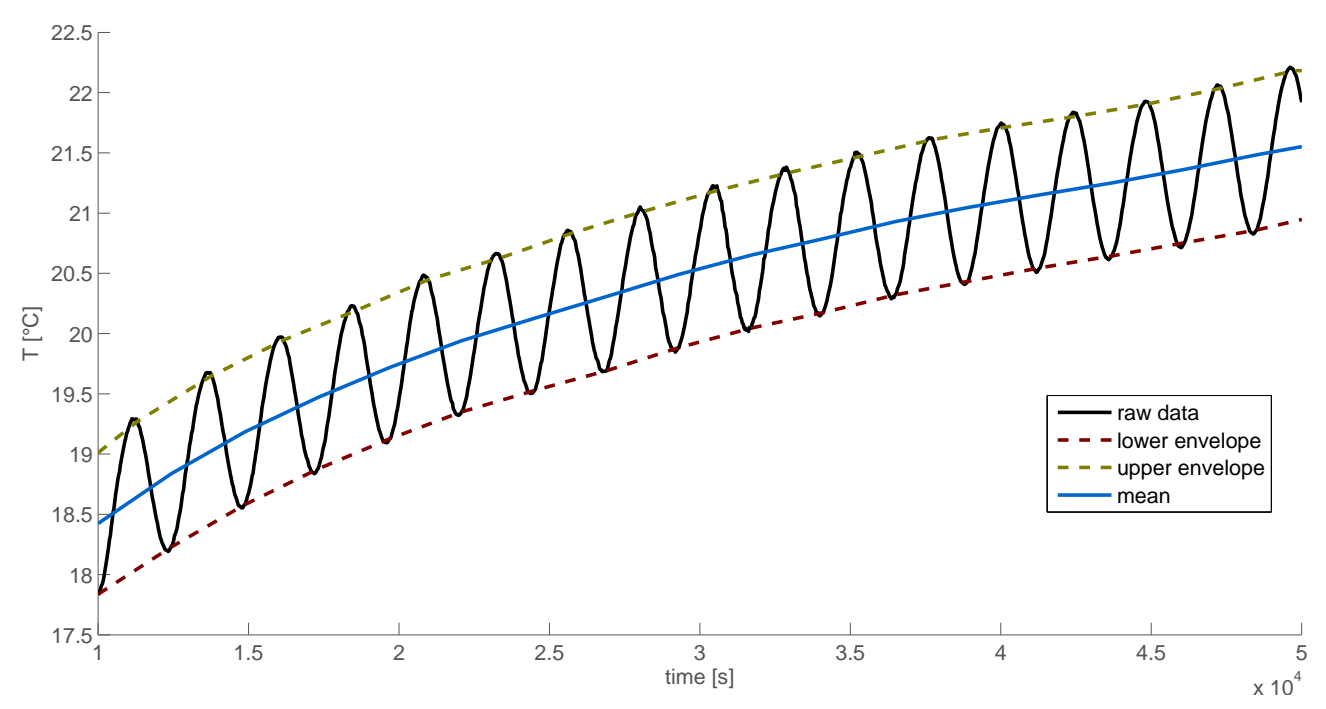

Figure C.1: Raw signal (black), envelopes (dashed) and mean of envelopes (blue)

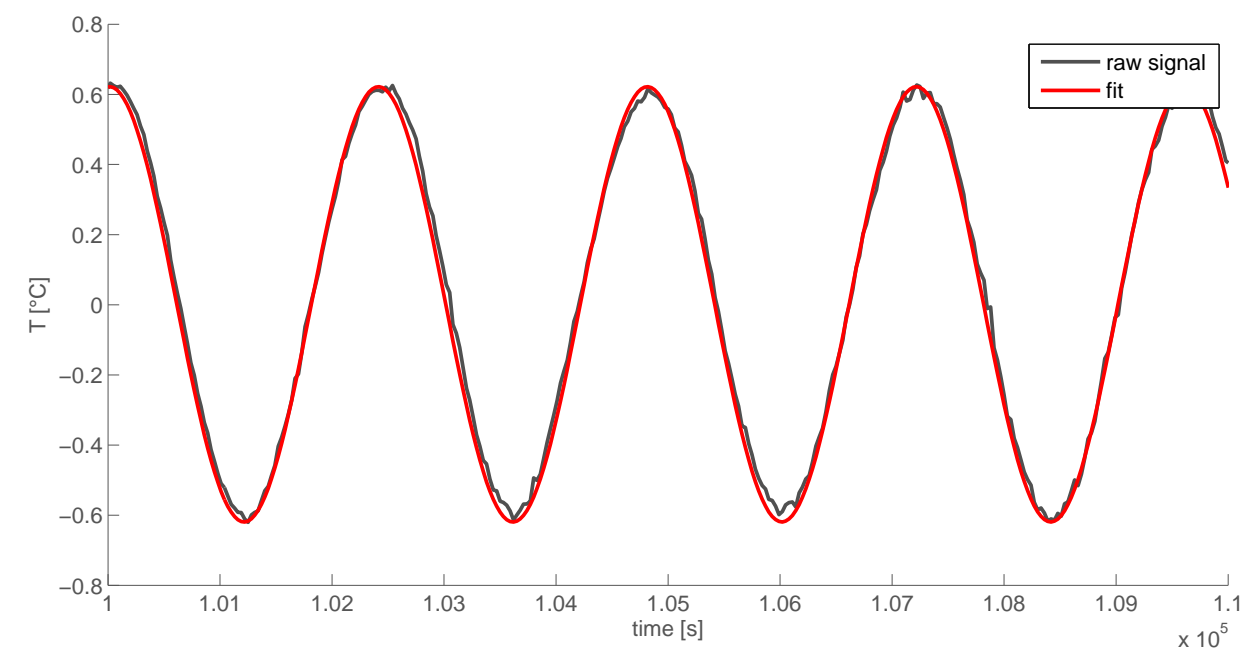

Figure C.2: Oscillatory heat injection rate $\dot{Q}_{1}(t)$, raw (black) and sine function fit (red)

and Mead [1965]). The wanted oscillatory signal response parameters, namely the amplitude and the phase shift, are gained from the fitting procedure. 


\section{Bibliography}

M. Abramowitz and I. A. Stegun. Handbook of Mathematical Functions with Formulas, Graphs, and Mathematical Tables, chapter 5. Dover, New York, ninth dover printing, tenth gpo printing edition, 1964.

J. Acuña. Improvements of U-pipe Borehole Heat Exchangers. Master's thesis, KTH, Applied Thermodynamics and Refrigeration, 2010.

J. Acuña and B. Palm. Local Conduction Heat Transfer in U-pipe Borehole Heat Exchangers. In Proceedings of the European COMSOL Conference in Milan, 2009.

J. Acuña and B. Palm. Distributed temperature measurements on a multi-pipe coaxial borehole heat exchanger. In Proceedings of the 10th IEA Heat Pump Conference. Japan, 2011.

R. Al-Khoury and H. Diersch. Computational Modeling of Shallow Geothermal Systems. Multiphysics modeling. CRC Press, 2011. ISBN 9780415596275.

R. Al-Khoury, P. G. Bonnier, and R. B. J. Brinkgreve. Efficient finite element formulation for geothermal heating systems. Part I: steady state. International Journal for Numerical Methods in Engineering, 63(7):988-1013, 2005. ISSN 1097-0207. doi: 10.1002/nme.1313.

S. Atalla, A. El-Sharkawy, and F. Gasser. Measurement of thermal properties of liquids with an AC heated-wire technique. International Journal of Thermophysics, 2(2): 155-162, 1981. ISSN 0195-928X. doi: 10.1007/BF00503938.

W. Austin, C. Yavuzturk, and J. D. Spitler. Development of an In-Situ System for Measuring Ground Thermal Properties. ASHRAE Transactions, 106(1):365-379, 2000. 
K. Avila, D. Moxey, A. de Lozar, M. Avila, D. Barkley, and B. Hof. The Onset of Turbulence in Pipe Flow. Science, 333(6039):192-196, 2011. doi: 10.1126/science. 1203223.

D. Barkley. Simplifying the complexity of pipe flow. Phys. Rev. E, 84:016309, Jul 2011. doi: 10.1103/PhysRevE.84.016309.

D. Bauer. Zur thermischen Modellierung von Erdwärmesonden und ErdsondenWärmespeichern. $\quad \mathrm{PhD}$ thesis, Universität Stuttgart, Holzgartenstr. 16, 70174 Stuttgart, 2011.

J. V. Beck, C. St Clair, and B. Blackwell. Inverse heat conduction. John Wiley and Sons Inc., New York, NY, 1985.

R. A. Beier, M. D. Smith, and J. D. Spitler. Minimum Duration of In-Situ Tests on Vertical Boreholes. ASHRAE Transactions, 109(2):475-486, 2003.

R. A. Beier, M. D. Smith, and J. D. Spitler. Reference data sets for vertical borehole ground heat exchanger models and thermal response test analysis. Geothermics, 40 (1):79 - 85, 2011. ISSN 0375-6505.

M. A. Bernier. Pumping Design and Performance Modeling of Geothermal Heat Pump Systems-Ground-Coupled Heat Pump System Simulation. ASHRAE TransactionsAmerican Society of Heating Refrigerating Airconditioning Engin, 107(1):605-616, 2001.

BMZ. Bundesministerium für wirtschaftliche Zusammenarbeit und Entwicklung, Materialen 186 (Entwicklung braucht nachhaltige Energie), 2013. URL http://www.bmz.de/de/publikationen/reihen/infobroschueren_flyer/ infobroschueren/Materialie186.pdf.

F. Bozzoli, G. Pagliarini, S. Rainieri, and L. Schiavi. Estimation of soil and grout thermal properties through a tspep (two-step parameter estimation procedure) applied to trt (thermal response test) data. Energy, 36(2):839 - 846, 2011. ISSN 0360-5442. doi: http://dx.doi.org/10.1016/j.energy.2010.12.031.

C. Bringedal, I. Berre, and J. M. Nordbotten. Influence of natural convection in a porous medium when producing from borehole heat exchangers. Water Resources Research, 49(8):4927-4938, 2013. ISSN 1944-7973. doi: 10.1002/wrcr.20388. 
D. G. Cahill and R. O. Pohl. Thermal conductivity of amorphous solids above the plateau. Phys. Rev. B, 35:4067-4073, Mar 1987. doi: 10.1103/PhysRevB.35.4067.

M. Cardiff, T. Bakhos, P. K. Kitanidis, and W. Barrash. Aquifer heterogeneity characterization with oscillatory pumping: Sensitivity analysis and imaging potential. Water Resources Research, 49(9):5395-5410, 2013a. ISSN 1944-7973. doi: $10.1002 /$ wrcr. 20356 .

M. Cardiff, T. Bakhos, P. K. Kitanidis, and W. Barrash. Multi-frequency oscillatory hydraulic tomography: The use of steady-periodic signals for sensitivity analysis and inversion. Water Resources Research (submitted), 2013b.

H. Carslaw. Introduction to the mathematical theory of the conduction of heat in solids. Dover Pub., 1906.

H. Carslaw and J. Jaeger. Conduction of heat in solids. Oxford science publications. Clarendon Press, 1959.

A. Chiasson, U. of Wyoming. Department of Civil, and A. Engineering. Simulation and Design of Hybrid Geothermal Heat Pump Systems. University of Wyoming, 2007. ISBN 9780549216933.

A. D. Chiasson, S. J. Rees, and J. D. Spitler. A preliminary assessment of the effects of groundwater flow on closed-loop ground source heat pump systems. Technical report, Oklahoma State Univ., Stillwater, OK (US), 2000.

H.-J. Diersch, W. Rühaak, P. Schätzl, D. Bauer, and W. Heidemann. Numerical Modelling of Solar Heat Storage Using Large Arrays of Borehole Heat Exchangers. In Proceedings World Geothermal Congress 2010, 2010.

H.-J. Diersch, D. Bauer, W. Heidemann, W. Rühaak, and P. Schätzl. Finite element modeling of borehole heat exchanger systems: Part 1. Fundamentals. Computers and Geosciences, 37(8):1122 - 1135, 2011. ISSN 0098-3004. doi: http://dx.doi.org/10. 1016/j.cageo.2010.08.003.

F. Dittus and L. Boelter. Heat Transfer in Automobile Radiators of the Tubular Type. University of California publications in engineering: University of California. University of California Press, 1930. 
O. Edenhofer, R. Pichs-Madruga, Y. Sokona, K. Seyboth, P. Matschoss, S. Kadner, T. Zwickel, P. Eickemeier, G. Hansen, S. Schlömer, and C. von Stechow. IPCC Special Report on Renewable Energy Sources and Climate Change Mitigation. Technical report, Cambridge University Press, Cambridge, United Kingdom and New York, NY, USA, 2011. URL http://www.ipcc.ch/pdf/special-reports/srren/SRREN_ Full_Report.pdf.

C. Eklöf and S. Gehlin. TED-a mobile equipment for thermal response test: testing and evaluation. Master's thesis, Luleå tekniska universitet, 1996.

L. Eppelbaum and I. Kutasov. Pressure and temperature drawdown well testing: similarities and differences. Journal of Geophysics and engineering, 3(1):12, 2006.

P. Eskilson. Thermal Analysis of Heat Extraction Boreholes. Lund-MPh. Lund University Press, 1987. ISBN 9789179002985.

H. Fujii, H. Okubo, K. Nishi, R. Itoi, K. Ohyama, and K. Shibata. An improved thermal response test for U-tube ground heat exchanger based on optical fiber thermometers. Geothermics, 38(4):399 - 406, 2009. ISSN 0375-6505. doi: http://dx.doi.org/10.1016/ j.geothermics.2009.06.002.

A. Gando, D. Dwyer, R. McKeown, and C. Zhang. Partial radiogenic heat model for Earth revealed by geoneutrino measurements. Nature Geoscience, 4(9):647-651, 2011.

S. Gehlin and G. Hellström. Influence on thermal response test by groundwater flow in vertical fractures in hard rock. Renewable energy, 28(14):2221-2238, 2003.

V. Gnielinski. New equations for heat and mass transfer in turbulent pipe and channel flow. Int. Chem. Eng, 16(2):359-368, 1976.

A. Griesinger, K. Spindler, and E. Hahne. Periodisches Hitzdrahtverfahren zur Messung von Wärme- und Temperaturleitfähigkeit von geringen Stoffmengen. Heat and Mass Transfer, 32(6):419-425, 1997. ISSN 0947-7411. doi: 10.1007/s002310050140.

J. D. Groot, J. Kestin, and H. Sookiazian. Instrument to measure the thermal conductivity of gases. Physica, 75(3):454 - 482, 1974. ISSN 0031-8914. doi: http: //dx.doi.org/10.1016/0031-8914(74)90341-3. 
GtV Bundesverband Geothermie. GtV Bundesverband Geothermie homepage, 2013. URL http://www.geothermie.de/wissenswelt/geothermie/technologien/ oberflaechennahe-geothermie.html.

A.-M. Gustafsson. Thermal response test: numerical simulations and analyses. $\mathrm{PhD}$ thesis, Luleå tekniska universitet, 2006.

GVC-VDI. VDI-Wärmeatlas. Springer, 2006. ISBN 9783540322184.

J. Healy, J. de Groot, and J. Kestin. The theory of the transient hot-wire method for measuring thermal conductivity. Physica $B+C, 82(2): 392-408$, 1976. ISSN 03784363. doi: http://dx.doi.org/10.1016/0378-4363(76)90203-5.

A. Huber and O. Schuler. Berechnungsmodul für Erdwärmesonden: Schlußbericht. Bundesamt für Energiewirtschaft, 1997.

H. Huber. Experimentelle und numerische Untersuchungen zum Wärmetransportverhalten oberflächennaher, durchströmter Böden. $\mathrm{PhD}$ thesis, TU Darmstadt, Darmstadt, 2013.

IEA. International Energy Agency, 2013. URL http://www.iea.org/textbase/npsum/ weo2010sum.pdf.

F. P. Incropera, A. S. Lavine, and D. P. DeWitt. Fundamentals of heat and mass transfer. John Wiley \& Sons Incorporated, 2011.

L. R. Ingersoll. Heat conduction: with engineering, geological, and other applications. University of Wisconsin Press, 1954.

ISFH. EFRE Project Geo-Solar-WP (High-efficient heat pump systems with geothermal and solar thermal energy sources), 2010. URL http://www.isfh.de/institut_ solarforschung/geo-solar-waermepumpe.php?dm=1\&_l=1.

L. Jackson. Signals, systems, and transforms. Addison-Wesley series in electrical engineering. ADDISON WESLEY Publishing Company Incorporated, 1991. ISBN 9780201095890 .

S. Javed, P. Fahlén, and J. Claesson. Vertical ground heat exchangers: a review of heat flow models. In Effstock 2009-Stockholm, Sweden, 2009-06-14-17. Proceedings vol. CD-proceedings, 2009. 
S. P. Kavanaugh and K. D. Rafferty. Ground-source heat pumps: design of geothermal systems for commercial and institutional buildings. American Society of Heating, Refrigerating and Air-Conditioning Engineers, 1997.

T. Kohl and R. Hopkirk. FRACTure - a simulation code for forced fluid flow and transport in fractured, porous rock. Geothermics, 24(3):333-343, 1995.

T. Kölbel. Grundwassereinfluss auf Erdwä̈rmesonden: Gelaändeuntersuchungen und Modellrechnungen. PhD thesis, Karlsruher Inst. für Technologie, 2010.

C. Kuo. Determination of reservoir properties from sinusoidal and multirate flow tests in one or more wells. Old SPE Journal, 12(6):499-507, 1972.

S. Kutateladze and V. Borishanskii. A Concise Encyclopedia of Heat Transfer. Pergamon Press, 1966.

S. Lazzari and E. Zanchini. BHE Field Design by Superposition of Effects in Space and Time. In Proceedings of the European COMSOL Conference in Milan, 2012.

C. Lee, K. Lee, H. Choi, and H.-P. Choi. Characteristics of thermally-enhanced bentonite grouts for geothermal heat exchanger in South Korea. Science in China Series E: Technological Sciences, 53(1):123-128, 2010.

Z. Li and M. Zheng. Development of a numerical model for the simulation of vertical Utube ground heat exchangers. Applied Thermal Engineering, 29(56):920 - 924, 2009. ISSN 1359-4311. doi: http://dx.doi.org/10.1016/j.applthermaleng.2008.04.024.

G. P. Merker. Konvektive Wärmeübertragung. Springer-Verlag, 1987. ISBN 978-3-54016995-6.

P. Mogensen. Fluid to duct wall heat transfer in duct system heat storages. In Proceedings of the International Conference on Surface Heat Storage in Theory and Practice, pages 652-657, Sweden, 1983.

J. A. Nelder and R. Mead. A simplex method for function minimization. The computer journal, 7(4):308-313, 1965.

P. Oberdorfer. Oscillatory Thermal Response Test (OTRT) An Advanced Method for Gaining Thermal Properties of the Subsurface. In Proceedings of the European COMSOL Conference in Rotterdam, 2013. 
P. Oberdorfer, F. Maier, and E. Holzbecher. Comparison of Borehole Heat Exchangers (BHEs): State of the Art vs. Novel Design Approaches. Proceedings of the European COMSOL Conference in Stuttgart, 2011.

P. Oberdorfer, E. Holzbecher, R. Hu, T. Ptak, and M. Sauter. A Five Spot Well Cluster for Hydraulic and Thermal Tomography. In Proceedings of the 38th Workshop on Geothermal Reservoir Engineering, 2013a.

P. Oberdorfer, R. Hu, E. Holzbecher, and M. Sauter. A Coupled FEM Model for Numerical Simulation of Rechargeable Shallow Geothermal BHE Systems. In Proceedings of the 38th Workshop on Geothermal Reservoir Engineering, 2013b. URL https: //pangea. stanford.edu/ERE/pdf/IGAstandard/SGW/2013/Oberdorfer.pdf.

W. L. Oberkampf and C. J. Roy. Verification and validation in scientific computing, volume 5. Cambridge University Press Cambridge, 2010.

T. Oppelt, I. Riehl, and U. Gross. Modelling of the borehole filling of double U-pipe heat exchangers. Geothermics, 39(3):270-276, 2010.

A. V. Oppenheim, R. W. Schafer, J. R. Buck, et al. Discrete-time signal processing, volume 5. Prentice Hall Upper Saddle River, 1999.

P. Pärisch, M. Kirchner, W. Wetzel, S. Voss, and R. Tepe. Test System for the Investigation of the Synergy Potential of Solar Collectors and Borehole Heat Exchangers in Heat Pump Systems. In Proceedings of the Solar World Congress 2011, 2011.

P. Pärisch, O. Mercker, P. Oberdorfer, E. Bertram, R. Tepe, and G. Rockendorf. Shortterm optimization of TRNSYS models for borehole heat exchangers regarding the combination with solar heat. Renewable Energy (submitted), 2013.

S. Rainieri, F. Bozzoli, and G. Pagliarini. Modeling approaches applied to the thermal response test: A critical review of the literature. HVACESR Research, 17(6):977-990, 2011.

R. Rawlings and J. Sykulski. Ground source heat pumps: a technology review. Building Services Engineering Research and Technology, 20(3):119-129, 1999.

S. J. Rees and M. He. A three-dimensional numerical model of borehole heat exchanger heat transfer and fluid flow . Geothermics, 46(0):1 - 13, 2013. ISSN 0375-6505. doi: http://dx.doi.org/10.1016/j.geothermics.2012.10.004. 
Renewable Energy Policy Network. Renewables 2013 - Global Status Report, 2013. URL http://www.ren21. net/Portals/0/documents/Resources/GSR/2013/ GSR2013_lowres.pdf.

M. Riegger. Qualitätsuntersuchung von Erdwärmesonden im Realmaßstab. Internationales Anwenderforum Oberflächennahe Geothermie, 2011.

W. Ruan and W. T. Horton. Literature Review on the Calculation of Vertical Ground Heat Exchangers for Geothermal Heat Pump Systems. In Proceedings of the International High Performance Buildings Conference, 2010.

O. Sandberg, P. Andersson, and G. Backstrom. Heat capacity and thermal conductivity from pulsed wire probe measurements under pressure. Journal of Physics E: Scientific Instruments, 10(5):474, 1977.

I. Sass and C. Lehr. Improvements on the thermal response test evaluation applying the cylinder source theory. In Proceedings of the 36th Stanford Geothermal Workshop, Stanford, 2011.

J. Shonder and J. Beck. A new method to determine the thermal properties of soil formations from in situ field tests. Oak Ridge National Laboratory, Rept. ORNL/TM2000, 97, 2000.

S. Signorelli, S. Bassetti, D. Pahud, and T. Kohl. Numerical evaluation of thermal response tests. Geothermics, 36(2):141 - 166, 2007. ISSN 0375-6505. doi: 10.1016/j. geothermics.2006.10.006.

J. Spitler. Editorial: Ground-Source Heat Pump System Research - Past, Present, and Future. HvacERR Research, 11(2):165-167, 2005.

Terra Umweltwärmesonde GmbH. Terra Umweltwärmesonde GmbH homepage, 2011. URL http://http://www. waermesonde.de/.

C. V. Theis. The relation between the lowering of the piezometric surface and the rate and duration of discharge of a well using ground water storage. US Department of the Interior, Geological Survey, Water Resources Division, Ground Water Branch, 1935.

Tyfocor. Technical Information: Antifreeze and Anticorrosion Concentrate for Heating and Cooling Circuits. Medium for Ground Source Heat Pump Systems, 2013. URL http://www.tyfo.de/docs/TI-TYFOCOR_gb_2013.pdf. 
UN. Delivering on Energy: An overview of activities by UN-Energy and its members, 2010. URL http://www.un-energy.org/publications/ 169-delivering-on-energy.

UNEP. 21 Issues for the 21st Century: Result of the UNEP Foresight Process on Emerging Environmental Issues, 2012. URL http://www.unep.org/publications/ ebooks/foresightreport/Portals/24175/pdfs/Foresight_Report-21_Issues_ for_the_21st_Century.pdf.

UNEP. United Nations Environment Programme, 2014. URL www . unep.org.

S. Voss. Durchführung, Aus- und Bewertung experimenteller Untersuchungen am Erdsondenversuchsfeld des ISFH im Projekt GeoSolarWP. Master's thesis, Hochschule für Angewandte Wissenschaften Hamburg, 2011.

M. Weber. Das Allgemeine Ähnlichkeitsprinzip der Physik und sein Zusammenhang mit der Dimensionslehre und der Modellwissenschaft. In Jahrbuch der Schiffbautechnischen Gesellschaft, pages 274-354. Springer Berlin Heidelberg, 1930. ISBN 978-3-64290164-5.

H. J. Witte, G. J. Van Gelder, and J. Spitler. In situ measurement of ground thermal conductivity: The dutch perspective. Ashrae Transactions, 108(1):263-272, 2002.

J. Wołoszyn and A. Gołaś. Modelling of a borehole heat exchanger using a finite element with multiple degrees of freedom. Geothermics, 47:13-26, 2013.

H. Yang, P. Cui, and Z. Fang. Vertical-borehole ground-coupled heat pumps: a review of models and systems. Applied Energy, 87(1):16-27, 2010.

C. Yavuzturk and J. D. Spitler. A short time step response factor model for vertical ground loop heat exchangers. ASHRAE transactions, 105(2):475-485, 1999.

T. R. Young. Development, verification, and design analysis of the borehole fluid thermal mass model for approximating short term borehole thermal. $\mathrm{PhD}$ thesis, Oklahoma State University, 2004.

E. Zanchini, S. Lazzari, and A. Priarone. Long-term performance of large borehole heat exchanger fields with unbalanced seasonal loads and groundwater flow. Energy, 38(1): 66 - 77, 2012. ISSN 0360-5442. doi: http://dx.doi.org/10.1016/j.energy.2011.12.038.

Last access date of all URLs: 01/19/2014 


\section{Curriculum Vitae (short)}

Phillip Oberdorfer

Geoscience Centre of the University of Göttingen

Dept. Applied Geology

Goldschmidtstr. 3

37077 Göttingen, Germany

http://www.uni-goettingen.de/en/8483.html

Email: poberdo@gwdg.de

Born: 05/31/1983 in Göttingen

Nationality: German / Austrian

Marital status: unmarried

\section{Education}

Since $10 / 2011$

$02 / 2011$

PhD student, Georg-August-University Göttingen

Degree in physics, Georg-August-University Göttingen

\section{Work Experience}

Since $01 / 2014$

Technical support engineer, Comsol Multiphysics GmbH

$03 / 2011-12 / 2013$

Research associate, Georg-August-University Göttingen 